\title{
Synthesis of $\gamma$-Lactams by Formal Cycloadditions with Ketenes
}

Audrey Viceriat, Isabelle Marchand, Sébastien Carret, * and Jean-François Poisson*

\section{Supporting information}

I. General Experimental

II. Synthesis of ketenes

III. Synthesis of aziridines 3

III.1. $\quad \mathrm{N}$-Tosylaziridines 3

III.2. Synthesis of the $\mathbf{N}$-alkylaziridine 20 5

IV. Synthesis of $\gamma$-lactams 6

IV.1. Optimization of the reaction conditions 6

IV.2. General procedures 7

IV.3. By-Products 8

IV.4. Lactams 10

V. NMR spectra 22

VI. HPLC Data 58

VII. X-Ray crystallographic analysis for compouds (S)-3a and $3 u$ 59

\section{General Experimental}

Reactions were carried out under argon in oven-dried glassware. Standard inert atmosphere techniques were used in handling all air and moisture sensitive reagents.

Anhydrous tetrahydrofuran was distilled from sodium/benzophenone. Anhydrous diethyl ether and dichloromethane were obtained with a MBRAUN dry solvents device by filtration through activated aluminium oxide. Acetonitrile was distilled over $\mathrm{P}_{2} \mathrm{O}_{5}$. Anhydrous ethanol was obtained by distillation from $\mathrm{Mg} / \mathrm{l}_{2}$. Triethylamine, dimethylethylamine and titanium tetrachloride were distilled from $\mathrm{CaH}_{2}$ and amines were stored over $\mathrm{KOH}$. Commercial solution of MeLi (1.6 [M] in diethyl ether) and $n$-BuLi (1.6 [M] in hexane) were titrated by using salicylaldehyde phenylhydrazone prior to use. ${ }^{1}$ Commercial diiodomethane, chloroiodomethane, and 1-iodohexane were distilled from $\mathrm{CaCl}_{2}$ and stored over copper in dark bottles. Commercial carboxylic acids, oxalyl chloride were distilled before use and $p$-toluenesulfonamide was recrystallized from hot water, then from ethanol, before utilization.

\footnotetext{
${ }^{1}$ B. E. Love, E. G. Jones, J. Org. Chem. 1999, 64, 3755-3756.
} 
All olefins, tetrakis(acetonitrile) copper (I) hexafluorophosphate, anhydride lithium iodide, 1,1-diiodoethane and all other reagents were purchased in the higher quality and used without further purification.

Reactions were monitored by thin-layer chromatography (TLC) carried out on silica-gel plates Merck (Kieselgel $\left.60 \mathrm{~F}_{254}-0.2 \mathrm{~mm}\right)$, using ultraviolet light $(\lambda 254 \mathrm{~nm}$ ) as the visualizing agent and basic potassium permanganate solution $\left(\mathrm{KMNO}_{4}\right)$ in water and heat as developing agents. Silica gel (Merck Kieselgel Gerduran - 0.040-0.063 mm) was employed for flash column chromatography.

${ }^{1} \mathrm{H}$ NMR and ${ }^{13} \mathrm{C}$ NMR spectra were recorded at $298 \mathrm{~K}$ on either a Brücker AV300, AV400, AV500 MHz apparatus. All shifts for ${ }^{1} \mathrm{H}$ and ${ }^{13} \mathrm{C}$ spectra were referenced to the residual solvent peak $\left(\mathrm{CDCl}_{3} \delta_{\mathrm{H}} 7.26, \delta_{\mathrm{C}} 77.16\right)$, ${ }^{19} \mathrm{~F}$ spectra were referenced to $\mathrm{CF}_{3} \mathrm{CO}_{2} \mathrm{H}$ in $\mathrm{CDCl}_{3}\left(\delta_{\mathrm{F}}-75.39\right)$ and are reported in ppm. The following abbreviations were used to explain the multiplicities: $\mathrm{s}=$ singlet, $\mathrm{d}=$ doublet, $\mathrm{t}=$ triplet, $\mathrm{q}=$ quartet, $\mathrm{m}=$ multiplet, $\mathrm{dd}=$ doublet of doublet, $\mathrm{dt}=$ doublet of triplet, $d q=$ doublet of quartet, $t d=$ triplet of doublet, $\mathrm{ps}=$ pseudo, $\mathrm{br}=$ broad, $J=$ coupling constant in Hertz $(\mathrm{Hz})$, $\delta=$ chemical shift in ppm. When ambiguous, proton and carbon assignments were established through COSY, HSQC, NOESY, and/or DEPT experiments. Infrared spectra (IR) were recorded on a Nicolet Impact-400 Fourier transform infrared spectrometer using ATR (Attenuated Total Reflexion) modes. Mass spectra were recorded by using APCI or ESI techniques on a Brücker Esquire 3000+ or on an UHPLC/MS apparatus Agilent 1290 Infinity/6120 Quadrupole device. High-resolution mass spectra (HRMS) were performed on a Brüker Q-Tof maXis mass spectrometer by the "Fédération de Recherche ICOA/CBM" (FR2708) platform. Optical rotations [ $\alpha$ ] were measured on a Perkin-Elmer 341 polarimeter. Enantiomeric excess (ee) were determined by HPLC with on a HPLC/SFC (supercritical fluid chromatography) apparatus Agilent 1260/1290 Infinity/Aurora SFC system, using Chiralpak IA, IB, IC and ID analytical column. Melting points were measured in capillary tubes on a Büchi B-545 device and are uncorrected.

\section{Synthesis of ketenes}

Diphenylketene was prepared according to a literature procedure and all analytical data are in accordance with the published values. ${ }^{2}$ Cycloheptylketene and ethylphenylketene were prepared according to literature procedures and all analytical data are in accordance with the published values. ${ }^{3,4}$

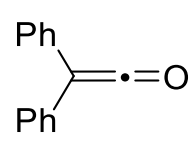

\section{Diphenylketene ; 2,2-Diphenylethen-1-one}

Triethylamine $(3.63 \mathrm{~mL}, 26.0 \mathrm{mmol}, 1.2 \mathrm{eq})$ was added via syringe over a 30 min period to a solution of 2,2diphenylacetyl chloride $(5.0 \mathrm{~g}, 21.6 \mathrm{mmol}, 1 \mathrm{eq})$ in $\operatorname{dry~}_{2} \mathrm{O}(30 \mathrm{~mL})$ at $0{ }^{\circ} \mathrm{C}$ under nitrogen atmosphere and the mixture was stirred overnight ( 16 hours) at $-20^{\circ} \mathrm{C}$. After warming to room temperature, ammonium salts were removed by filtration under argon and the filtrate was concentrated in vacuo. Distillation under reduced pressure at $80^{\circ} \mathrm{C}(0.04$

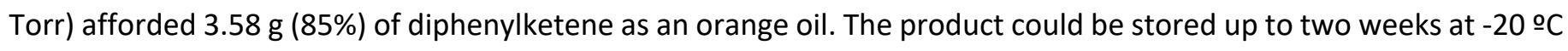
in the freezer before substantial decomposition occurred.

IR $\boldsymbol{v}_{\max }$ (neat): 3056, 2094, 1660, 1501, $1430 \mathrm{~cm}^{-1}$.

\footnotetext{
${ }^{2}$ J. M. Goll, E. Fillion, Organometallics 2008, 27, 3622-3625.

${ }^{3}$ B. L. Hodous, G. C. Fu, J. Am. Chem. Soc. 2002, 124, 1578-1579.

${ }^{4}$ L. M. Baigrie, H. R. Seiklay, T. T. Tidwell, J. Am. Chem. Soc. 1985, 107, 5391-5396.
} 
${ }^{1} \mathrm{H}$ NMR (400 MHz, $\left.\mathrm{CDCl}_{3}\right): \delta(\mathrm{ppm})$ 7.40-7.37 (m, 5H), 7.28-7.24 (m, 5H).

${ }^{13} \mathrm{C} \mathrm{NMR}\left(75 \mathrm{MHz}, \mathrm{CDCl}_{3}\right): \delta$ (ppm) 201.1, 130.8, 129.2, 127.7, 126.2, 46.8.

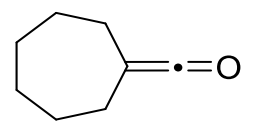

\section{Cycloheptylketene; Cycloheptylidenemethanone}

To a stirred solution of cycloheptanoyl chloride $(3.0 \mathrm{~g}, 18.6 \mathrm{mmol}, 1 \mathrm{eq})$ in dry $\mathrm{Et}_{2} \mathrm{O}(70 \mathrm{~mL})$ under argon was added dimethylethylamine $(2.77 \mathrm{~mL}, 20.54 \mathrm{mmol}, 1.1 \mathrm{eq}$ ) via syringe over a 30 min period. The reaction was then stirred for 40 hours at room temperature. After this time, the reaction was filtered under argon to remove ammonium salts. The $\mathrm{Et}_{2} \mathrm{O}$ was then removed under reduced pressure to give a yellow oil which was purified via distillation at $55^{\circ} \mathrm{C}$ ( 5 Torr) to afford $928 \mathrm{mg}(40 \%)$ of cycloheptylketene as a yellow liquid. The product could be stored at $-20{ }^{\circ} \mathrm{C}$ in the freezer and was used rapidly.

IR $\boldsymbol{v}_{\max }$ (neat): 2923, 2851, 2095, 1523, $1247 \mathrm{~cm}^{-1}$.

${ }^{1} \mathrm{H}$ NMR (400 MHz, $\left.\mathrm{CDCl}_{3}\right): \delta(\mathrm{ppm})$ 2.25-2.22 (m, 4H), 1.76-1.62 (m, 4H), 1.60-1.51 (m, 4H).

MS (APCI) $m / z 125.1[\mathrm{M}+\mathrm{H}]^{+}$.<smiles>CC/C(=C\C=O)c1ccccc1</smiles>

\section{Ethylphenylketene ; 2-Phenylbut-1-en-1-one}

Triethylamine $(15.3 \mathrm{~mL}, 110.0 \mathrm{mmol}, 4 \mathrm{eq})$ was added via syringe over a 30 min period to a solution of 2phenylbutanoyl chloride $(5.0 \mathrm{~g}, 27.2 \mathrm{mmol}, 1$ eq.) in dry THF $(55 \mathrm{~mL})$ at room temperature under argon atmosphere and the mixture was stirred for $3 \mathrm{~h} 30$. Ammonium salts were removed by filtration under argon and the filtrate was concentrated in vacuo. Distillation under reduced pressure at $70{ }^{\circ} \mathrm{C}(0.5 \mathrm{Torr})$ afforded $1.59 \mathrm{~g}(40 \%)$ of phenylethylketene as a yellow oil. The product can be stored under argon in freezer at $-20^{\circ} \mathrm{C}$ for one week without significant dimerization.

IR $\boldsymbol{v}_{\max }$ (neat): $2971,2878,2084,1597,1496 \mathrm{~cm}^{-1}$.

${ }^{1} \mathrm{H}$ NMR $\left(400 \mathrm{MHz}, \mathrm{CDCl}_{3}\right): \delta(\mathrm{ppm}) 7.31$ (ps t, $\left.J=8.0 \mathrm{~Hz}, 3 \mathrm{H}\right), 7.08-7.02(\mathrm{~m}, 2 \mathrm{H}), 2.44(\mathrm{q}, J=7.2 \mathrm{~Hz}, 2 \mathrm{H}), 1.23(\mathrm{t}, J=7.2$ $\mathrm{Hz}, 3 \mathrm{H})$.

\section{III.Synthesis of aziridines}

\section{III.1. $N$-Tosylaziridines}

- $\mathbf{N}$-tosylimine $\mathbf{1}$ have been prepared following reported procedures $s^{5,6}: \mathbf{1} \mathbf{a}, \mathbf{b}, \mathbf{c}, \mathbf{d}, \mathbf{f}, \mathbf{g}, \mathbf{h}, \mathbf{i}, \mathbf{j}, \mathbf{I}$ are prepared according reference $5 ; \mathbf{1 e}, \mathbf{k}, \mathbf{n}$ are prepared according reference 6 . Analytical data are in accordance with published values. $^{7}$

\footnotetext{
${ }^{5}$ C. Verrier, S. Carret, J.-F. Poisson, ACS Sustain. Chem. Eng. 2018, 6, 8563-8569.

${ }^{6}$ F. Chemla, V. Hebbe, J.-F. Normant, Synthesis 2000, 75-77.

${ }^{7}$ Reference 6 and : (a) Z. Li, X. Ren, P. Wei, H. Wan, Y. Shi, P. Ouyang, Green Chem. 2006, 8, 433M. (b) Barbarotto, J. Geist, S. Choppin, F. Colobert, Tetrahedron Asymmetry 2009, 20, 2780-2787 (c) C.-J. Wang, M. Shi, J. Org. Chem. 2003, 68, 6229-6237 (d) J. L. García Ruano, J. Alemán, C. Fajardo, A. Parra, Org. Lett. 2005, 7, 5493-5496 (e) W. B. Jennings, C. J. Lovely, Tetrahedron Lett. 1988, 29, 3725-3728 (f) T. Jin, G. Feng, M. Yang, T. Li, Synth. Commun. 2004, 34, 1277-1283.
} 
- $\quad \mathbf{N}$-tosylaziridine $\mathbf{2 a - I}$ and $\mathbf{2 n}$ have been prepared according to modified literature procedures for optimization of [3+2] cycloaddition of ketenes, ${ }^{8,9}$ and all analytical data are in accordance with the published values. 2-(prop-1-en-2-yl)-1-tosylaziridine $\mathbf{2 m}$ was prepared according Kim's procedure. ${ }^{10}$

General Procedure for the synthesis of $\boldsymbol{N}$-tosylaziridines ( $2 \mathrm{a}-\mathrm{I}$ and $\mathbf{2 n}$ ) from imines (1a-I and $\mathbf{1 n})$.

To a mixture of the requisite $N$-tosylimine 1 (1 eq) and $\mathrm{CH}_{2} \mathrm{I}_{2}$ (2 eq) in dry THF $(0.172 \mathrm{M})$ was added dropwise a solution of MeLi in ether (1.6 [M], 2 eq) over 30 minutes, at $-78{ }^{\circ} \mathrm{C}$ under argon. The solution was stirred at the same temperature for 45 minutes and then was left to stir at room temperature for an additional 45 minutes. The reaction mixture was then quenched with saturated aqueous $\mathrm{NH}_{4} \mathrm{Cl}$, and the organic layer was then extracted three times with diethyl ether. The organic layers were washed with saturated aqueous $\mathrm{NaCl}$ solution and dried over $\mathrm{Na}_{2} \mathrm{SO}_{4}$, filtered and concentrated in vacuo. Flash chromatography on silica gel (eluent EtOAc/Pentane, 10:90 to 30:70) of the crude mixture afforded the pure $\mathrm{N}$-tosylaziridine $\mathbf{2}$.

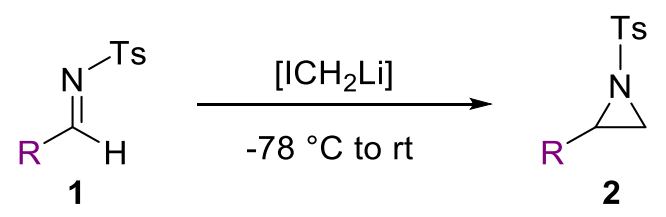

\begin{tabular}{c|c|c} 
Number & $\mathbf{R}$ & Isolated yield (\%) \\
\hline $2 \mathrm{a}$ & $\mathrm{Ph}$ & 80 \\
\hline $2 \mathrm{~b}$ & $p \mathrm{~F}-\mathrm{Ph}$ & 85 \\
\hline $2 \mathrm{c}$ & $p \mathrm{Br}-\mathrm{Ph}$ & 78 \\
\hline $2 \mathrm{~d}$ & $p \mathrm{Me}-\mathrm{Ph}$ & 25 \\
\hline $2 \mathrm{e}$ & $p$ NO2-Ph & 57 \\
\hline $2 \mathrm{f}$ & $m \mathrm{~F}-\mathrm{Ph}$ & 76 \\
\hline $2 \mathrm{~g}$ & $m \mathrm{Br}-\mathrm{Ph}$ & 82 \\
\hline $2 \mathrm{~h}$ & $m \mathrm{MeO}-\mathrm{Ph}$ & 86 \\
\hline $2 \mathrm{i}$ & $o$ Me-Ph & 85 \\
\hline $2 \mathrm{j}$ & Naphtyl & 90 \\
\hline $2 \mathrm{l}$ & Cinnamyl & 80 \\
\hline $2 \mathrm{n}$ & Cyclohexyl & 62
\end{tabular}

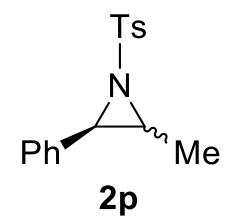

\section{2-Methyl-3-phenyl-1-tosylaziridine (2p)}

To a mixture of the $N$-tosylimine $2 \mathrm{a}$ ( $230 \mathrm{mg}, 0.887 \mathrm{mmol}, 1 \mathrm{eq}$ ) and 1,1-diiodoethane $(0.179 \mathrm{~mL}, 1.77 \mathrm{mmol}, 2 \mathrm{eq})$ in dry THF ( $5 \mathrm{~mL}, 0.172 \mathrm{M}$ ) was added dropwise a solution of MeLi in ether (1.10 mL, 1.6 [M], $1.16 \mathrm{mmol}, 2$ eq) over 30 minutes, at $-78^{\circ} \mathrm{C}$ under argon. The solution was stirred at the same temperature for 45 minutes and then was left to stir at room temperature for an additional 45 minutes. The reaction mixture was then quenched with saturated aqueous $\mathrm{NH}_{4} \mathrm{Cl}$, and the organic layer was then extracted three times with diethyl ether. The organic layers were washed with saturated aqueous $\mathrm{NaCl}$ solution and dried over $\mathrm{Na}_{2} \mathrm{SO}_{4}$, filtered and concentrated in vacuo. Flash chromatography on silica gel (eluent EtOAc/Pentane, 10:90 to 30:70) of the crude mixture afforded $210 \mathrm{mg}(82 \%)$ of $N$-tosylaziridine $\mathbf{2 p}$ as a $73: 27$ ( $\mathbf{2 p}$ trans:2p cis) mixture of diastereoisomer as a colourless oil.

\footnotetext{
${ }^{8}$ Z. Li, K. R. Conser, E. N. Jacobsen, J. Am. Chem. Soc. 1993, 115, 5326-5327.

${ }^{9}$ (a) J. M. Concellón, H. Rodríguez-Solla, P. L. Bernad, C. Simal, J. Org. Chem. 2009, 74, 2452-2459 (b) J. M. Concellón, H. Rodríguez-Solla, C. Simal, Org. Lett. 2008, 10, 4457-4460.

${ }^{10}$ A. B. Smith, D.-S. Kim, J. Org. Chem. 2006, 71, 2547-2557.
} 


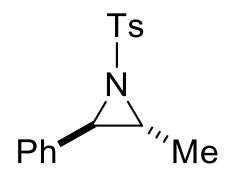

$2 p$ trans

\section{trans-2-Methyl-3-phenyl-1-tosylaziridine (2p-trans)}

For analysis, a small pure sample of $\mathbf{1 8 4 a}$ can be isolated by flash chromatography on silica gel (eluent EtOAc/Pentane, 10:90 to $30: 70$ ) as a colourless oil.

${ }^{1} \mathrm{H}$ NMR $\left(400 \mathrm{MHz}, \mathrm{CDCl}_{3}\right): \delta(\mathrm{ppm}) 7.82(\mathrm{~d}, J=8.5 \mathrm{~Hz}, 2 \mathrm{H}), 7.27-7.23(\mathrm{~m}, 5 \mathrm{H}), 7.16-7.13(\mathrm{~m}, 2 \mathrm{H}), 3.79(\mathrm{~d}, J=4.5 \mathrm{~Hz}$, $1 \mathrm{H}), 2.91(\mathrm{dq}, J=6.0,4.5 \mathrm{~Hz}, 1 \mathrm{H}), 2.39(\mathrm{~s}, 3 \mathrm{H}), 1.84(\mathrm{~d}, J=6.0 \mathrm{~Hz}, 3 \mathrm{H})$.

${ }^{13} \mathrm{C}$ NMR (125 MHz, CDCl $): \delta$ (ppm) 144.0, 138.1, 135.8, 129.9, 129.7, 128.7, 128.2, 127.4, 126.5, 49.3, 21.7, 14.3.

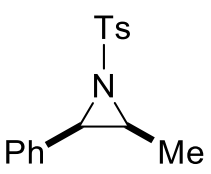

$2 p$ cis

cis-2-Methyl-3-phenyl-1-tosylaziridine (2p-cis)

Following the procedure $\boldsymbol{B}$ of aziridination: A solution of $\left[\mathrm{Cu}\left(\mathrm{CH}_{3} \mathrm{CN}\right)_{4}\right] \mathrm{PF}_{6}(11.7 \mathrm{mg}, 0.031 \mathrm{mmol}, 10.6 \mathrm{~mol} \%)$ and ligand diamine 216a (14 mg, $0.032 \mathrm{mmol}, 11 \mathrm{~mol} \%)$ in dry $\mathrm{CH}_{2} \mathrm{Cl}_{2}(2 \mathrm{~mL})$ was stirred under argon for 1 hour at room temperature. cis- $\beta$-methylstyrene $(76 \mu \mathrm{L}, 0.590 \mathrm{mmol}, 2 \mathrm{eq})$ was added and the flask was then cooled to $-78^{\circ} \mathrm{C}$. Solid $\mathrm{Phl}=\mathrm{NTs} 225(110 \mathrm{mg}, 0.295 \mathrm{mmol}, 1 \mathrm{eq})$ was added against a positive argon flow. The solution was allowed to warm to room temperature over the night. Then, the solution was quenched with $\mathrm{H}_{2} \mathrm{O}$, and the product was extracted three times with $\mathrm{CH}_{2} \mathrm{Cl}_{2}$. The organic layers were washed with saturated aqueous $\mathrm{NaCl}$ solution and dried over $\mathrm{Na}_{2} \mathrm{SO}_{4}$, filtered and concentrated in vacuo to give $N$-tosylaziridine $\mathbf{1 8 4}$ as a 86:14 (184b cis:184a trans) mixture of diastereoisomer as a colourless oil. Flash chromatography on silica gel (eluent EtOAc/Pentane, 5:95 to 30:70) of the crude mixture afforded $47 \mathrm{mg}(55 \%)$ of cis- $\mathrm{N}$-tosylaziridine $184 \mathrm{~b}$ as a colourless oil.

IR $\boldsymbol{v}_{\text {max }}$ (neat): $2968,2926,1598,1454,1305,1289,1151,1087,969,838 \mathrm{~cm}^{-1}$.

${ }^{1} \mathrm{H}$ NMR $\left(300 \mathrm{MHz}, \mathrm{CDCl}_{3}\right): \delta(\mathrm{ppm}) 7.89(\mathrm{~d}, J=8.4 \mathrm{~Hz}, 2 \mathrm{H}), 7.34(\mathrm{~d}, J=8.1 \mathrm{~Hz}, 2 \mathrm{H}), 7.29-7.24(\mathrm{~m}, 3 \mathrm{H}), 7.23-7.16(\mathrm{~m}$, $2 \mathrm{H}), 3.93(\mathrm{~d}, J=7.2 \mathrm{~Hz}, 1 \mathrm{H}), 3.19(\mathrm{dq}, J=7.2,5.7 \mathrm{~Hz}, 1 \mathrm{H}), 2.44(\mathrm{~s}, 3 \mathrm{H}), 1.02(\mathrm{~d}, J=5.7 \mathrm{~Hz}, 3 \mathrm{H})$.

${ }^{13} \mathrm{C}$ NMR (125 MHz, CDCl $)$ : $\delta$ (ppm) 129.9, 128.4, 127.98, 127.7, 46.2, 41.7, 21.8, 12.1.

Quaternary carbon (CAr) was not seen.

MS (APCI) $m / z 288.1[\mathrm{M}+\mathrm{H}]^{+}$.

\section{III.2. Synthesis of the $N$-alkylaziridine 20}

$\mathrm{N}$-alkylaziridine $\mathbf{2 0}$ was prepared according to literature procedures ${ }^{11}$ and all analytical data are in accordance with the published values. The following synthesis have not been optimized.

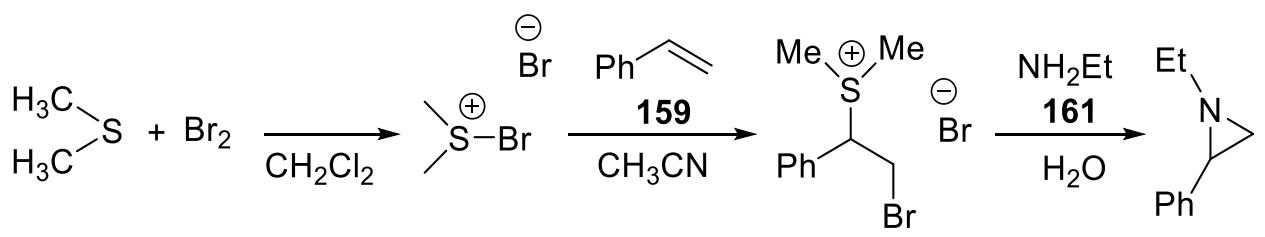

\footnotetext{
${ }^{11}$ Y. Du, Y. Wu, A.-H. Liu, L.-N. He, J. Org. Chem. 2008, 73, 4709-4712.
} 


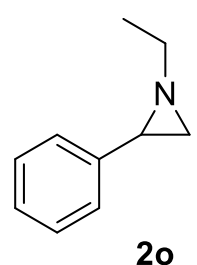

\section{1-Ethyl-2-phenylaziridine (20)}

To a stirred solution of styrene sulphonium bromide $(2.0 \mathrm{~g}, 6.1 \mathrm{mmol}, 1 \mathrm{eq}$.) in $12.5 \mathrm{~mL}$ of water at room temperature, a solution of ethanamine in water $(1.99 \mathrm{~mL}, 24.5 \mathrm{mmol}, 4 \mathrm{eq}, 70 \% \mathrm{Wt})$ was added dropwise and the resulting mixture was stirred overnight. The mixture was poured into $12.5 \mathrm{~mL}$ of saturated aqueous $\mathrm{NaCl}$, extracted with diethyl ether $(3 \times 12 \mathrm{~mL})$, dried with $\mathrm{Na}_{2} \mathrm{SO}_{4}$, filtered and the solvent evaporated under reduced pressure. Pure aziridine $2 \mathrm{o}$ was obtained without purification $(0.858 \mathrm{~g}, 95 \%)$ as a yellow oil.

IR $\boldsymbol{v}_{\max }$ (neat): 3037, 2967, 2829, 1495, 1447, 1375, 1208, 1176, 1079, $734 \mathrm{~cm}^{-1}$.

${ }^{1} \mathrm{H}$ NMR $\left(400 \mathrm{MHz}, \mathrm{CDCl}_{3}\right): \delta(\mathrm{ppm})$ 7.31-7.19 (m, 5H), 2.51-2.29 (m, 2H), $2.30(\mathrm{dd}, J=6.4,3.2 \mathrm{~Hz}, 1 \mathrm{H}), 1.89(\mathrm{~d}, J=3.2$ $\mathrm{Hz}, 1 \mathrm{H}), 1.65(\mathrm{~d}, J=6.4 \mathrm{~Hz}, 1 \mathrm{H}), 1.20(\mathrm{t}, J=6.8 \mathrm{~Hz}, 3 \mathrm{H}$ ).

${ }^{13} \mathrm{C}$ NMR (100 MHz, CDCl $): \delta$ (ppm) 140.7, 128.4, 126.9, 126.3, 56.6, 41.3, 37.7, 14.6.

MS (APCI) $m / z 148.1[\mathrm{M}+\mathrm{H}]^{+}$.

\section{IV.Synthesis of Y-lactams}

\section{IV.1. Optimization of the reaction conditions}

\section{Lewis acids as additive}

To a stirred solution of $\mathrm{N}$-tosylaziridine 2 (1 eq) and Lewis acid $(1 \mathrm{eq})$ in $\mathrm{dry} \mathrm{CH}_{2} \mathrm{Cl}_{2}(0.1[\mathrm{M}])$ at $-78^{\circ} \mathrm{C}$ was slowly added via syringe diphenylketene (12) (2 eq) in a dry $\mathrm{CH}_{2} \mathrm{Cl}_{2}(0.3[\mathrm{M}])$, under argon. The reaction iswas allowed to warm to room temperature and was stirred until disappearance of the starting aziridine (TLC). The solution was quenched with saturated aqueous $\mathrm{NaHCO}_{3}$ solution, and the product was extracted three times with EtOAc. The organic layers were washed with saturated aqueous $\mathrm{NaCl}$ solution and dried over $\mathrm{Na}_{2} \mathrm{SO}_{4}$, filtered and concentrated in vacuo.

- Using $\mathrm{Sc}(\mathrm{OTf})_{3}, \mathrm{Zn}(\mathrm{OTf})_{2}, \mathrm{Sn}(\mathrm{OTf})_{2}$ or $\mathrm{BF}_{3} . \mathrm{Et}_{2} \mathrm{O}$, ketene dimers and very complex mixture were obtained, with total consumption of starting aziridine and no lactame product observed.

- Using $\mathrm{Nil}_{2}$, starting aziridine was recovered and no lactame formation was observed.

\section{Halide salts as additive in THF}

To a stirred solution of $N$-tosylaziridine $\mathbf{2}(1 \mathrm{eq})$ and additive ( 1 eq, or $\mathrm{X}$ eq see table) in dry THF (0.2 [M]) was slowly added via syringe diphenylketene (12) (2 eq) in a dry THF (0.4 [M]) over 1 hour, under argon. After being, stirred 2 hours at room temperature, the solution was quenched with saturated aqueous $\mathrm{NaHCO}_{3}$ solution, and the product was extracted three times with EtOAc. The organic layers were washed with saturated aqueous $\mathrm{NaCl}$ solution and dried over $\mathrm{Na}_{2} \mathrm{SO}_{4}$, filtered and concentrated in vacuo.

\begin{tabular}{llll}
\hline Entry & Additive & $\begin{array}{l}\text { Conv } \\
{[\%]^{[b]}}\end{array}$ & Lactam : Oxazolidine \\
\hline 1 & Nal & 98 & $79: 21$ \\
2 & LiCl & 0 & $0: 0$ \\
& LiBr & 60 & $77: 23$ \\
3 & BTEAC & 0 & $0: 0$ \\
4 & BTEAC $+\mathrm{BF}_{3} \cdot \mathrm{Et}_{2} \mathrm{O}$ & 100 & $0: 0$ \\
5 & $n \mathrm{Bu}_{4} \mathrm{NI}$ & 80 & $75: 25$
\end{tabular}




\begin{tabular}{llll}
6 & $n \mathrm{Bu}_{4} \mathrm{NI}+\mathrm{BF}_{3} \cdot \mathrm{Et}_{2} \mathrm{O}$ & $/^{[\mathrm{e}]}$ & Degradation products \\
7 & $\begin{array}{l}\text { Lil (rapide addition of } \\
\text { ketene) }\end{array}$ & 100 & $75: 25$ \\
8 & Lil & 98 & $>95: 5(80 \%)$ \\
9 & Lil (0,1 eq) & 45 & $>95: 5$ \\
10 & Lil (0,2 eq) & 76 & $>95: 5$ \\
11 & Lil (0,5 eq) & 90 & $>95: 5$ \\
12 & Lil (0,8 eq) & 100 & $>95: 5(83 \%)$ \\
\hline
\end{tabular}

\section{Solvents study with lithium iodide}

To a stirred solution of $N$-tosylaziridine $\mathbf{2}(1 \mathrm{eq})$ and lithium iodide (1 eq) in dry solvent (0.2 [M]) was slowly added via syringe diphenylketene (12) (2 eq) in a dry solvent (0.4 [M]) over 1 hour, under argon. After being, stirred 2 hours at room temperature, the solution was quenched with saturated aqueous $\mathrm{NaHCO}_{3}$ solution, and the product was extracted three times with EtOAc. The organic layers were washed with saturated aqueous $\mathrm{NaCl}$ solution and dried over $\mathrm{Na}_{2} \mathrm{SO}_{4}$, filtered and concentrated in vacuo.

\begin{tabular}{lllll}
\hline Entry & Solvent & Aziridine & $\begin{array}{l}\text { Lactam } \\
\text { Oxazolidine }\end{array}$ & $\begin{array}{l}\text { lsolated } \\
\text { Yield }\end{array}$ \\
\hline 1 & THF & 2 & $>95: 5$ & 80 \\
2 & $\mathrm{CH}_{2} \mathrm{Cl}_{2}$ & 0 & $>95: 5$ & 70 \\
3 & $\mathrm{Et}_{2} \mathrm{O}$ & 0 & $>95: 5$ & 82 \\
4 & Toluene & 0 & $92: 8$ & 76 \\
\hline
\end{tabular}

\section{IV.2. General procedures}

General procedure A for the synthesis of $y$-lactams, according to the $(3+2)$ cycloaddition To a stirred solution of $N$-tosylaziridine 2 ( 1 eq) and lithium iodide (0.8 eq) in dry THF (0.2 [M]) was slowly added via syringe diphenylketene (12) (2 eq) in a dry THF (0.4 [M]) over 1 hour, under argon. After being, stirred 2 hours at room temperature, the solution was quenched with saturated aqueous $\mathrm{NaHCO}_{3}$ solution, and the product was extracted three times with EtOAc. The organic layers were washed with saturated aqueous $\mathrm{NaCl}$ solution and dried over $\mathrm{Na}_{2} \mathrm{SO}_{4}$, filtered and concentrated in vacuo. Flash chromatography on silica gel (eluent EtOAc/Pentane, 5:95 to 30:70) of the crude mixture gave the pure $\mathrm{\gamma}$-lactam $\mathbf{3}$.

General procedure $\mathbf{B}$ for the synthesis of $y$-lactams, according to the $(2+1+2)$ cycloaddition from imines

To a solution of $N$-tosylimine 1 (1 eq) in dry THF (0.2 [M]) was added diiodomethane (2 eq), under argon. The solution was allowed to stir at $-78^{\circ} \mathrm{C}$, and was added dropwise a solution of methyllithium in ether (1.6 [M], 2 eq) over 30 minutes. The solution was allowed to stir for 1 hour at $-78{ }^{\circ} \mathrm{C}$, further 30 minutes at room temperature. Then, diphenylketene ( 2 eq) in dry THF (0.4 [M]) was slowly added via syringe over 1 hour. After the resulting mixture has been stirred for 2 hours, it was quenched with saturated aqueous $\mathrm{NaHCO}_{3}$ solution, extracted three times with EtOAc. The combined organic layers were washed with saturated aqueous $\mathrm{NaCl}$ solution and dried over anhydrous $\mathrm{Na}_{2} \mathrm{SO}_{4}$, filtered, and the filtrate was concentrated under reduced pressure. The crude material was then purified by flash chromatography on silica gel (eluent EtOAc/Pentane, 5:95 to 30:70) to afford the pure $\psi$-lactam 3. 


\section{IV.3. By-Products}

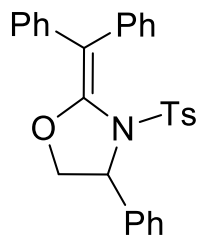

4a

\section{2-(Diphenylmethylene)-4-phenyl-3-tosyloxazolidine (4a)}

By product of the $(3+2)$ cycloaddition reaction. For analysis, a small pure sample of $4 a$ can be isolated by flash chromatography on silica gel (eluent EtOAc/Pentane, 20:80) as a white solid.

m.p. $154-155^{\circ} \mathrm{C}$.

IR $v_{\max }$ (neat): 3056, 2920, 2853, 1742, 1647, 1492, 1494, 1359, 1164, $698 \mathrm{~cm}^{-1}$.

${ }^{1} \mathrm{H}$ NMR $\left(500 \mathrm{MHz}, \mathrm{CDCl}_{3}\right): \delta(\mathrm{ppm}) 7.56(\mathrm{~d}, J=8.5 \mathrm{~Hz}, 2 \mathrm{H}), 7.40-7.35(\mathrm{~m}, 3 \mathrm{H}), 7.33-7.32(\mathrm{~m}, 2 \mathrm{H}), 7.28-7.24(\mathrm{~m}, 5 \mathrm{H})$, 7.22-7.18 (m, 2H), 7.12-7.09 (m, 3H), 6.98-6.96 (m, 2H), $5.36(\mathrm{~d}, J=5.5 \mathrm{~Hz}, 1 \mathrm{H}), 4.25(\mathrm{dd}, J=8.5,1.5 \mathrm{~Hz}, 1 \mathrm{H}), 3.90$ (dd, $J=8.5,6.5 \mathrm{~Hz}, 1 \mathrm{H}), 2.43(\mathrm{~s}, 3 \mathrm{H})$.

${ }^{13} \mathrm{C}$ NMR (125 MHz, CDCl 3 ): $\delta$ (ppm) 144.7, 140.4, 139.9, 138.4, 135.3, 130.8, 130.1, 129.9, 129.3, 129.1, 128.7, 128.0, $127.9,127.1,126.8,126.4,112.2,70.4,67.1,62.3,21.8$.

MS (ESI) $m / z$ 467.9 $[\mathrm{M}+\mathrm{H}]^{+}$.

HRMS (ESI) m/z: $[\mathrm{M}+\mathrm{H}]^{+}$Calcd for $\mathrm{C}_{29} \mathrm{H}_{26} \mathrm{NO}_{3} \mathrm{~S}$ 468.1628; Found 468.1630.<smiles>ICC(N[I-])Pc1ccccc1</smiles>

\section{$\mathbf{N}$-(2-lodo-1-phenylethyl)-4-methylbenzenesulfonamide ${ }^{12}$}

By product of the [3+2] cycloaddition reaction. For analysis, a small pure sample of $\mathbf{1 4 8}$ can be isolated by flash chromatography on silica gel (eluent EtOAc/Pentane, 20:80) as a white solid.

m.p. $126-127^{\circ} \mathrm{C}$ [litt $\left.126-128^{\circ} \mathrm{C}\right]$.

IR $v_{\max }$ (neat): $3269,3030,1600,1456,1328,1160,1092,701,669 \mathrm{~cm}^{-1}$.

${ }^{1} \mathrm{H}$ NMR $\left(300 \mathrm{MHz}, \mathrm{CDCl}_{3}\right): \delta(\mathrm{ppm}) 7.64(\mathrm{~d}, J=8.4 \mathrm{~Hz}, 2 \mathrm{H}), 7.26-7.20(\mathrm{~m}, 5 \mathrm{H}), 7.11-7.08(\mathrm{~m}, 2 \mathrm{H}), 5.05(\mathrm{br} \mathrm{d}, J=6.6 \mathrm{~Hz}$, $1 \mathrm{H}), 4.40$ (ps q, $J=6.6 \mathrm{~Hz}, 1 \mathrm{H}), 3.48-3.38(\mathrm{~m}, 2 \mathrm{H}), 2.40(\mathrm{~s}, 3 \mathrm{H})$.

${ }^{13} \mathrm{C}$ NMR (125 MHz, $\left.\mathrm{CDCl}_{3}\right): 143.8,138.6,137.0,129.7,129.0,128.9,128.5,127.4,126.6,58.1,21.7,11.7$.

MS (APCl) $m / z 274.1[\mathrm{M}-1]^{+}$.<smiles>INCC(I)c1ccccc1</smiles>

$\mathbf{N}$-(2-lodo-2-phenylethyl)-4-methylbenzenesulfonamide ${ }^{13}$

By product of the [3+2] cycloaddition reaction. For analysis, a small pure sample of $\mathbf{1 6 7}$ can be isolated by flash chromatography (eluent EtOAc/Pentane, 20:80).

IR $v_{\max }$ (neat): $3280,2920,1597,1454,1329,1328,1159,1092 \mathrm{~cm}^{-1}$.

${ }^{1} \mathrm{H}$ NMR $\left(400 \mathrm{MHz}, \mathrm{CDCl}_{3}\right): \delta(\mathrm{ppm}) 7.72(\mathrm{~d}, J=8.4 \mathrm{~Hz}, 2 \mathrm{H}), 7.33(\mathrm{~d}, J=8.4 \mathrm{~Hz}, 2 \mathrm{H}), 7.30-7.23(\mathrm{~m}, 5 \mathrm{H}), 5.02(\mathrm{t}, J=7.6$ $\mathrm{Hz}, 1 \mathrm{H}), 4.67(\mathrm{t}, J=6.4 \mathrm{~Hz}, 1 \mathrm{H}), 3.74-3.67(\mathrm{~m}, 1 \mathrm{H}), 3.55-3.47(\mathrm{~m}, 1 \mathrm{H}), 2.46(\mathrm{~s}, 3 \mathrm{H})$.

${ }^{13} \mathrm{C}$ NMR (125 MHz, CDCl $)$ ): 144.0, 140.0, 137.2, 130.0, 129.3, 129.0, 127.7, 127.2, 51.5, 30.0, 21.7.

MS (ESI) $m / z 424.1[\mathrm{M}+\mathrm{Na}]^{+}$.

\footnotetext{
${ }^{12}$ S. Minakata, J. Hayakawa, Chem. Commun. 2011, 47, 1905-1907.

${ }^{13}$ M. K. Ghorai, A. Kumar, D. P. Tiwari, J. Org. Chem. 2010, 75, 137-151.
} 


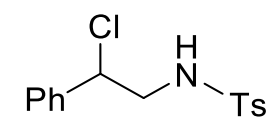

$\mathbf{N}$-(2-Chloro-2-phenylethyl)-4-methylbenzenesulfonamide

By product of the [3+2] cycloaddition reaction. For analysis, a small pure sample of $\mathbf{5}$ can be isolated by flash chromatography on silica gel (eluent EtOAc/Pentane, 20:80).

IR $\boldsymbol{v}_{\text {max }}$ (neat): $3279,2922,1494,1329,1158,1093,698 \mathrm{~cm}^{-1}$.

${ }^{1} \mathrm{H}$ NMR $\left(500 \mathrm{MHz}, \mathrm{CDCl}_{3}\right): \delta(\mathrm{ppm}) 7.65(\mathrm{~d}, J=8.2 \mathrm{~Hz}, 2 \mathrm{H}), 7.29-7.18(\mathrm{~m}, 7 \mathrm{H}), 4.79(\mathrm{dd}, J=8.5,6.0 \mathrm{~Hz}, 1 \mathrm{H}), 4.73(\mathrm{t}, J=$ $6.0 \mathrm{~Hz}, 1 \mathrm{H}), 3.43-3.32(\mathrm{~m}, 2 \mathrm{H}), 2.36(\mathrm{~s}, 3 \mathrm{H})$.

${ }^{13}$ C NMR (125 MHz, CDCl $)$ ): 144.0, 137.9, 137.1, 130.0, 129.6, 129.1, 127.3, 127.2, 61.8, 50.5, 21.7.

MS (APCl) $m / z 310.0[\mathrm{M}+\mathrm{H}]^{+}, 274.0[\mathrm{M}-\mathrm{Cl}]^{+}$. 


\section{IV.4. Lactams}

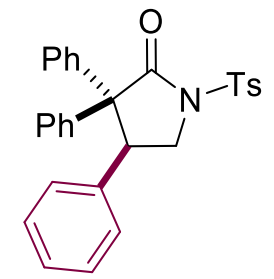

$3 a$

\section{3,3,4-Triphenyl-1-tosylpyrrolidin-2-one (3a)}

Procedure A: aziridine $2 \mathrm{a}(60 \mathrm{mg}, 0.219 \mathrm{mmol})$ and diphenylketene $(85 \mathrm{mg}, 0.439 \mathrm{mmol})$ afforded $85 \mathrm{mg}(83 \%)$ of $\gamma^{-}$ lactam $3 a$ as a foam solid.

Procedure B: imine 1a (60 mg, $0.232 \mathrm{mmol})$ and diphenylketene $(90 \mathrm{mg}, 0.463 \mathrm{mmol})$ afforded $95 \mathrm{mg}(88 \%)$ of $\gamma$-lactam 3a as a foam solid.

Procedure B for gram-scale production: imine $1 \mathrm{a}(1.0 \mathrm{~g}, 3.9 \mathrm{mmol})$ and diphenylketene $(1.5 \mathrm{~g}, 7.8 \mathrm{mmol})$ afforded 1.5 $\mathrm{g}(83 \%)$ of $\mathrm{Y}$-lactam $3 \mathrm{a}$ as a foam solid.

m.p. $172-173^{\circ} \mathrm{C}$.

IR $\boldsymbol{V}_{\text {max }}$ (neat): $3058,2922,1738,1596,1496,1367,1172 \mathrm{~cm}^{-1}$.

${ }^{1} \mathrm{H}$ NMR $\left(400 \mathrm{MHz}, \mathrm{CDCl}_{3}\right): \delta(\mathrm{ppm}) 7.92(\mathrm{~d}, J=8.4 \mathrm{~Hz}, 2 \mathrm{H}), 7.39-7.36(\mathrm{~m}, 2 \mathrm{H}), 7.32(\mathrm{~d}, J=8.4 \mathrm{~Hz}, 2 \mathrm{H}), 7.28-7.24(\mathrm{~m}$, $3 \mathrm{H}), 7.14-7.10(\mathrm{~m}, 1 \mathrm{H}), 7.06-7.02(\mathrm{~m}, 3 \mathrm{H}), 6.91(\mathrm{ps} \mathrm{t}, J=7.6 \mathrm{~Hz}, 2 \mathrm{H}), 6.75(\mathrm{~d}, J=7.2 \mathrm{~Hz}, 2 \mathrm{H}), 6.46(\mathrm{~d}, J=7.6 \mathrm{~Hz}, 2 \mathrm{H}), 4.44$ (ps t, $J=6.8 \mathrm{~Hz}, 1 \mathrm{H}), 4.22(\mathrm{dd}, J=10.0,6.8 \mathrm{~Hz}, 1 \mathrm{H}), 4.00(\mathrm{dd}, J=10.0,6.8 \mathrm{~Hz}, 1 \mathrm{H}), 2.48(\mathrm{~s}, 3 \mathrm{H})$.

${ }^{13} \mathrm{C} \mathrm{NMR}\left(100 \mathrm{MHz}, \mathrm{CDCl}_{3}\right): \delta$ (ppm) 173.8, 145.5, 139.4, 138.8, 137.1, 134.7, 129.8, 129.5, 129.2, 128.9, 128.6, 128.5, $128.52,128.46,128.3,127.8,127.7,127.6,127.0,64.3,49.5,47.0,21.9$.

MS (ESI) $\mathrm{m} / \mathbf{z} 490.3[\mathrm{M}+\mathrm{Na}]^{+}$.

HRMS (ESI) $\mathrm{m} / \mathrm{z}:[\mathrm{M}+\mathrm{H}]^{+}$calcd for $\mathrm{C}_{29} \mathrm{H}_{26} \mathrm{NO}_{3} \mathrm{~S}$ 468.1628; Found 468.1630.

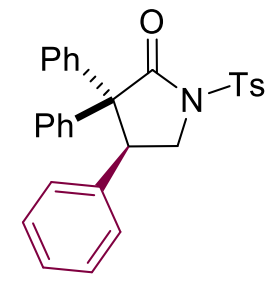

$(-)-3 a$

(S)-3,3,4-Triphenyl-1-tosylpyrrolidin-2-one (-)-3a

Procedure A: aziridine (-)-2a $(60 \mathrm{mg}, 0.219 \mathrm{mmol})$ and diphenylketene $(85 \mathrm{mg}, 0.439 \mathrm{mmol})$ afforded $86 \mathrm{mg}(84 \%)$ of $\gamma$-lactam (-)-3a as a foam solid. Analytical and spectroscopic data are the same as the racemic $\mathbf{3 a}$.

$[\alpha]^{24}{ }_{D}-93.8$ (c 1.0, $\left.\mathrm{CHCl}_{3}\right)$.

ee 97\%; HPLC (Chiralpak IB, $s \mathrm{CO}_{2}$-methanol 80:20, flow rate $=1.7 \mathrm{~mL} / \mathrm{min}, \lambda=220 \mathrm{~nm}$ ) tR $=7.15 \mathrm{~min}$ (major), $7.75 \mathrm{~min}$ (minor).<smiles>O=C1N([125I])CC(c2ccc(F)cc2)C1(c1ccccc1)c1ccccc1</smiles>

$3 b$

4-(4-Fluorophenyl)-3,3-diphenyl-1-tosylpyrrolidin-2-one (3b) 
Procedure A: aziridine $\mathbf{2 b}(60 \mathrm{mg}, 0.206 \mathrm{mmol})$ and diphenylketene $\left(80 \mathrm{mg}, 0.412 \mathrm{mmol}\right.$ ) afforded $97 \mathrm{mg}(97 \%)$ of $\mathrm{Y}^{-}$ lactam $\mathbf{3 b}$ as a foam solid.

Procedure B: imine $1 \mathrm{~b}(60 \mathrm{mg}, 0.216 \mathrm{mmol})$ and diphenylketene $(84 \mathrm{mg}, 0.432 \mathrm{mmol})$ afforded $85 \mathrm{mg}(81 \%)$ of $\gamma^{-}$ lactam $\mathbf{3 b}$ as a foam solid.

m.p. $82-83^{\circ} \mathrm{C}$.

IR $v_{\text {max }}$ (neat): $3060,2891,1733,1597,1508,1444,1356,1116,969 \mathrm{~cm}^{-1}$.

${ }^{1} \mathrm{H}$ NMR (400 MHz, $\left.\mathrm{CDCl}_{3}\right): \delta(\mathrm{ppm}) 7.91(\mathrm{~d}, J=8.4 \mathrm{~Hz}, 2 \mathrm{H}), 7.37-7.31(\mathrm{~m}, 4 \mathrm{H}), 7.26-7.25(\mathrm{~m}, 4 \mathrm{H}), 7.06(\mathrm{ps} \mathrm{t}, J=7.2 \mathrm{~Hz}$, $1 \mathrm{H}), 6.93(\mathrm{ps} \mathrm{t}, J=8.0 \mathrm{~Hz}, 2 \mathrm{H}), 6.77-6.68(\mathrm{~m}, 3 \mathrm{H}), 6.45(\mathrm{~d}, J=7.2 \mathrm{~Hz}, 2 \mathrm{H}), 4.43(\mathrm{ps} \mathrm{t}, J=6.8 \mathrm{~Hz}, 1 \mathrm{H}), 4.21(\mathrm{dd}, J=10.0$, $6.8 \mathrm{~Hz}, 1 \mathrm{H}), 3.95$ (dd, $J=10.0,6.8 \mathrm{~Hz}, 1 \mathrm{H}), 2.48(\mathrm{~s}, 3 \mathrm{H})$.

$\left.{ }^{13} \mathrm{C} \mathrm{NMR} \mathrm{(125} \mathrm{MHz,} \mathrm{CDCl} 3\right): \delta(\mathrm{ppm})$ 173.6, $163.1(\mathrm{C}-\mathrm{F}, 1 \mathrm{JC}-\mathrm{F}=246 \mathrm{~Hz}), 161.1$ (C-F, 1JC-F = $\left.246 \mathrm{~Hz}\right), 145.6,139.0,138.6$, 134.5, $132.83(C-F, 4 J C-F=3.0 \mathrm{~Hz}), 132.81(C-F, 4 J C-F=3.0 \mathrm{~Hz}), 130.5(C-F, 3 J C-F=8.0 \mathrm{~Hz}), 130.4(C-F, 3 J C-F=8.0 \mathrm{~Hz})$, $129.8,129.4,128.6,128.48,128.44,127.9,127.8,127.2,115.3(C-F, 2 J C-F=21.1 \mathrm{~Hz}), 115.1(C-F, 2 J C-F=21.1 \mathrm{~Hz}), 64.2$, 49.5, 46.2, 21.9 .

${ }^{19} \mathrm{~F}$ NMR (376 MHz, $\left.\mathrm{CDCl}_{3}\right): \delta(\mathrm{ppm})-114.0$

MS (ESI) $m / z 485.9[\mathrm{M}+\mathrm{H}]^{+}, 507.9[\mathrm{M}+\mathrm{Na}]^{+}$.

HRMS (ESI) $\mathrm{m} / \mathbf{z}[\mathrm{M}+\mathrm{H}]^{+}$calcd for $\mathrm{C}_{29} \mathrm{H}_{25} \mathrm{FNO}_{3} \mathrm{~S}$ 486.1534; Found 486.1535 .

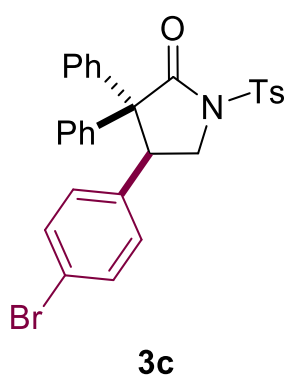

\section{4-(4-Bromophenyl)-3,3-diphenyl-1-tosylpyrrolidin-2-one (3c)}

Procedure A: aziridine $2 \mathrm{c}(55 \mathrm{mg}, 0.156 \mathrm{mmol})$ and diphenylketene $\left(61 \mathrm{mg}, 0.312 \mathrm{mmol}\right.$ ) afforded $84 \mathrm{mg}(98 \%)$ of $\mathrm{Y}^{-}$ lactam $3 \mathbf{c}$ as a foam solid.

Procedure B: imine 1c (78 mg, $0.232 \mathrm{mmol})$ and diphenylketene $(90 \mathrm{mg}, 0.463 \mathrm{mmol})$ afforded $110 \mathrm{mg}(87 \%)$ of $\gamma^{-}$ lactam $3 \mathrm{c}$ as a white solid.

m.p. $191-192^{\circ} \mathrm{C}$.

IR $v_{\max }$ (neat): 3067, 2892, 1728, 1597, 1494, 1366, 1160, 1087, $707 \mathrm{~cm}^{-1}$.

${ }^{1} \mathrm{H}$ NMR $\left(500 \mathrm{MHz} \mathrm{CDCl}_{3}\right): \delta(\mathrm{ppm}) 7.91(\mathrm{~d}, J=8.0 \mathrm{~Hz}, 2 \mathrm{H}), 7.36-7.32(\mathrm{~m}, 4 \mathrm{H}), 7.29-7.25(\mathrm{~m}, 3 \mathrm{H}), 7.17(\mathrm{~d}, J=8.5 \mathrm{~Hz}$, 2H), 7.07 (ps t, $J=7.5 \mathrm{~Hz}, 1 \mathrm{H}$ ), $6.94($ ps t, $J=8.0 \mathrm{~Hz}, 2 \mathrm{H}), 6.60(\mathrm{~d}, J=8.5 \mathrm{~Hz}, 2 \mathrm{H}), 6.47(\mathrm{~d}, J=7.5 \mathrm{~Hz}, 2 \mathrm{H}), 4.40$ (ps t, $J=$ $6.5 \mathrm{~Hz}, 1 \mathrm{H}$ ), 4.20 (dd, $J=10.0,6.5 \mathrm{~Hz}, 1 \mathrm{H}), 3.92(\mathrm{dd}, J=10.0,6.5 \mathrm{~Hz}, 1 \mathrm{H}), 2.48(\mathrm{~s}, 3 \mathrm{H})$.

${ }^{13} \mathrm{C} \mathrm{NMR}\left(125 \mathrm{MHz}, \mathrm{CDCl}_{3}\right): \delta$ (ppm) 173.5, 145.6, 139.0, 138.5, 136.2, 134.5, 131.4, 130.5, 129.8, 129.4, 128.6, 128.43, $128.42,127.9,127.3,121.7,64.0,49.4,46.5,21.9$.

MS (ESI) $m / z 545.7[\mathrm{M}+\mathrm{H}]^{+}, 567.7[\mathrm{M}+\mathrm{Na}]^{+}$.

HRMS (ESI) $\mathrm{m} / \mathrm{z}[\mathrm{M}+\mathrm{H}]^{+}$calcd for $\mathrm{C}_{29} \mathrm{H}_{25} \mathrm{BrNO}_{3} \mathrm{~S} 546.0733$; Found 546.0732.

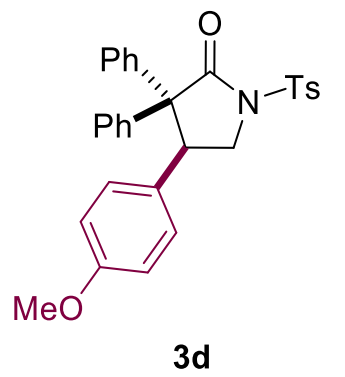

4-(4-Methoxyphenyl)-3,3-diphenyl-1-tosylpyrrolidin-2-one (144e)

Procedure B: imine 1d $(67 \mathrm{mg}, 0.232 \mathrm{mmol})$ and diphenylketene $(90 \mathrm{mg}, 0.463 \mathrm{mmol}$ ) afforded after purification by flash chromatography on silica gel $\left(\mathrm{SiO}_{2} / 2.5 \% \mathrm{Et}_{3} \mathrm{~N}\right), 86 \mathrm{mg}(75 \%)$ of $\gamma$-lactam $\mathbf{3 d}$ as a foam solid. 
m.p. $177-178^{\circ} \mathrm{C}$.

IR $\boldsymbol{v}_{\max }$ (neat): $3053,2902,1733,1514,1496,1356,1250,1168 \mathrm{~cm}^{-1}$.

${ }^{1} \mathbf{H}$ NMR (400 MHz, CDCl 3$): \delta(p p m) 7.93(\mathrm{~d}, J=8.0 \mathrm{~Hz}, 2 \mathrm{H}), 7.38-7.32(\mathrm{~m}, 4 \mathrm{H}), 7.27-7.24(\mathrm{~m}, 3 \mathrm{H}), 7.06(\mathrm{ps} \mathrm{t}, J=7.2 \mathrm{~Hz}$, $1 \mathrm{H}), 6.93$ (ps t, $J=8.4 \mathrm{~Hz}, 2 \mathrm{H}), 6.65-6.58(\mathrm{~m}, 4 \mathrm{H}), 6.44(\mathrm{~d}, J=7.6 \mathrm{~Hz}, 2 \mathrm{H}), 4.41(\mathrm{ps} \mathrm{t}, J=7.2 \mathrm{~Hz}, 1 \mathrm{H}), 4.19(\mathrm{dd}, J=9.8,7.2$ $\mathrm{Hz}, 1 \mathrm{H}), 3.92(\mathrm{dd}, J=9.8,7.2 \mathrm{~Hz}, 1 \mathrm{H}), 3.71(\mathrm{~s}, 3 \mathrm{H}), 2.48(\mathrm{~s}, 3 \mathrm{H})$.

${ }^{13} \mathrm{C}$ NMR (125 MHz, $\mathrm{CDCl}_{3}$ ): $\delta$ (ppm) 174.0, 159.0, 145.5, 139.5, 138.8, 134.7, 130.1, 129.8, 129.5, 128.7, 128.5, 128.4, 127.7, 127.1, 113.6, 64.2, 55.3, 49.6, 46.3, 21.9.

MS (ESI) $m / z 498.2[\mathrm{M}+\mathrm{H}]^{-}$.

HRMS (ESI) m/z [M+H] $]^{+}$calcd for $\mathrm{C}_{30} \mathrm{H}_{28} \mathrm{NO}_{4} \mathrm{~S}$ 498.1734; Found 498.1732.<smiles>[3H]N1CC(c2ccc([N+](=O)[O-])cc2)C(c2ccccc2)(c2ccccc2)C1=O</smiles>

$3 e$

4-(4-Nitrophenyl)-3,3-diphenyl-1-tosylpyrrolidin-2-one (3e)

Procedure A: aziridine 2 e $(52.6 \mathrm{mg}, 0.165 \mathrm{mmol})$ and diphenylketene $(64.2 \mathrm{mg}, 0.330 \mathrm{mmol})$ afforded after purification by flash chromatography on silica gel $\left(\mathrm{SiO}_{2} / 2.5 \% \mathrm{Et}_{3} \mathrm{~N}\right), 80 \mathrm{mg}(94 \%)$ of $\gamma$-lactam $3 e$ as a foam solid.

Procedure B: imine 1e (70.5 $\mathrm{mg}, 0.232 \mathrm{mmol})$ and diphenylketene $(90 \mathrm{mg}, 0.463 \mathrm{mmol})$ afforded after purification by flash chromatography on silica gel $\left(\mathrm{SiO}_{2} / 2.5 \% \mathrm{Et}_{3} \mathrm{~N}\right), 56 \mathrm{mg}(47 \%)$ of $\gamma$-lactam $3 e$ as a foam solid.

m.p. $83-84^{\circ} \mathrm{C}$.

IR $\boldsymbol{V}_{\max }$ (neat): $3065,1730,1597,1492,1445,1340,1169 \mathrm{~cm}^{-1}$.

${ }^{1} \mathrm{H}$ NMR (500 MHz, CDCl $): \delta(p p m) 7.89(\mathrm{dd}, J=8.5,5.5 \mathrm{~Hz}, 3 \mathrm{H}), 7.36-7.26(\mathrm{~m}, 9 \mathrm{H}), 7.05(\mathrm{ps} \mathrm{t}, J=7.5 \mathrm{~Hz}, 1 \mathrm{H}), 6.95-6.93$ $(\mathrm{m}, 3 \mathrm{H}), 6.50(\mathrm{~d}, J=8.0 \mathrm{~Hz}, 2 \mathrm{H}), 4.53(\mathrm{ps} \mathrm{t}, J=6.0 \mathrm{~Hz}, 1 \mathrm{H}), 4.25(\mathrm{dd}, J=10.0,6.0 \mathrm{~Hz}, 1 \mathrm{H}), 4.03(\mathrm{dd}, J=10.0,6.0 \mathrm{~Hz}, 1 \mathrm{H})$, $2.48(\mathrm{~s}, 3 \mathrm{H})$.

${ }^{13} \mathrm{C}$ NMR (125 MHz, $\left.\mathrm{CDCl}_{3}\right): \delta$ (ppm) 172.9, 147.2, 145.8, 145.2, 138.4, 138.3, 134.3, 129.9, 129.7, 129.2, 128.8, 128.4, $128.2,128.1,127.5,123.4,64.1,49.1,46.7,21.8$.

MS (ESI) $m / z 511.1[\mathrm{M}-\mathrm{H}]^{+}$.

HRMS (ESI) $\mathrm{m} / \mathrm{z}[\mathrm{M}+\mathrm{H}]^{+}$calcd for $\mathrm{C}_{29} \mathrm{H}_{25} \mathrm{~N}_{2} \mathrm{O}_{5} \mathrm{~S}$ 513.1479; Found 513.1480.

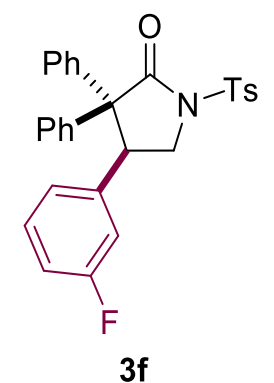

\section{4-(3-Fluorophenyl)-3,3-diphenyl-1-tosylpyrrolidin-2-one (3f)}

Procedure A: aziridine $\mathbf{2 f}$ ( $79 \mathrm{mg}, 0.271 \mathrm{mmol}$ ) and diphenylketene (105 $\mathrm{mg}, 0.542 \mathrm{mmol}$ ) afforded $118 \mathrm{mg}(90 \%)$ of $\gamma$ lactam $3 f$ as a foam solid.

Procedure B: imine 1f (65 mg, $0.234 \mathrm{mmol}$ ) and diphenylketene (91 mg, $0.469 \mathrm{mmol})$ afforded $81 \mathrm{mg}(71 \%)$ of $\mathrm{\gamma}$-lactam $3 f$ as a foam solid.

m.p. $176-177^{\circ} \mathrm{C}$.

IR $\boldsymbol{V}_{\max }$ (neat): $3059,1733,1591,1492,1366,1164,1126,981 \mathrm{~cm}^{-1}$.

${ }^{1} \mathrm{H}$ NMR (400 MHz, $\left.\mathrm{CDCl}_{3}\right): \delta(\mathrm{ppm}) 7.92(\mathrm{~d}, J=8.4 \mathrm{~Hz}, 2 \mathrm{H}), 7.38-7.32(\mathrm{~m}, 4 \mathrm{H}), 7.28-7.26(\mathrm{~m}, 3 \mathrm{H}), 7.08-7.00(\mathrm{~m}, 2 \mathrm{H})$, 6.93(ps t, $J=7.6 \mathrm{~Hz}, 2 \mathrm{H}$ ), 6.81 (ps td, $J=8.2,2.0 \mathrm{~Hz}, 1 \mathrm{H}), 6.61(\mathrm{~d}, J=8.0 \mathrm{~Hz}, 1 \mathrm{H}), 6.48(\mathrm{~d}, J=7.2 \mathrm{~Hz}, 2 \mathrm{H}), 6.34(\mathrm{ps} \mathrm{dt}, J$ $=7.6,2.0 \mathrm{~Hz}, 1 \mathrm{H}), 4.43(\mathrm{pst}, J=6.4 \mathrm{~Hz}, 1 \mathrm{H}), 4.23(\mathrm{dd}, J=10.0,6.4 \mathrm{~Hz}, 1 \mathrm{H}), 3.96(\mathrm{dd}, J=10.0,6.4 \mathrm{~Hz}, 1 \mathrm{H}), 2.48(\mathrm{~s}, 3 \mathrm{H})$. 
$\left.{ }^{13} \mathrm{C} \mathrm{NMR} \mathrm{(125} \mathrm{MHz,} \mathrm{CDCl}\right)$ ): $\delta$ (ppm) 173.5, $163.5(\mathrm{C}-\mathrm{F}, 1 \mathrm{JC}-\mathrm{F}=245 \mathrm{~Hz}), 161.50$ (C-F, 1JC-F = $\left.245 \mathrm{~Hz}\right), 145.7,139.8$ (C-F, $2 \mathrm{JC}-\mathrm{F}=7.5 \mathrm{~Hz}$ ), $139.7(\mathrm{C}-\mathrm{F}, 2 \mathrm{JC}-\mathrm{F}=7.5 \mathrm{~Hz}), 138.9,138.5,134.4,129.9,129.8,129.2,128.6,128.37$ (C-F, 3JC-F = 2.5 Hz), $128.35(\mathrm{C}-\mathrm{F}, 3 \mathrm{JC}-\mathrm{F}=2.5 \mathrm{~Hz}$ ), 127.9, 127.8, 127.2, $124.52(\mathrm{C}-\mathrm{F}, 4 \mathrm{JC}-\mathrm{F}=2.5 \mathrm{~Hz}), 124.49(\mathrm{C}-\mathrm{F}, 4 \mathrm{JC}-\mathrm{F}=2.5 \mathrm{~Hz}), 115.9$ (C-F, $2 \mathrm{JC}-\mathrm{F}=22.5 \mathrm{~Hz}), 115.7(\mathrm{C}-\mathrm{F}, 2 \mathrm{JC}-\mathrm{F}=22.5 \mathrm{~Hz}), 114.6(\mathrm{C}-\mathrm{F}, 2 \mathrm{JC}-\mathrm{F}=22.5 \mathrm{~Hz}), 114.4(\mathrm{C}-\mathrm{F}, 2 \mathrm{JC}-\mathrm{F}=22.5 \mathrm{~Hz}), 64.2,49.3,46.6$, 21.9.

$\left.{ }^{19} \mathrm{~F} \mathrm{NMR} \mathrm{(376} \mathrm{MHz,} \mathrm{CDCl}_{3}\right): \delta(\mathrm{ppm})-112.5$

MS (ESI) $m / z 508.0[\mathrm{M}+\mathrm{Na}]^{+}$.

HRMS (ESI) $\mathrm{m} / \mathrm{z}[\mathrm{M}+\mathrm{H}]^{+}$calcd for $\mathrm{C}_{29} \mathrm{H}_{25} \mathrm{FNO}_{3} \mathrm{~S}$ 486.1534; Found 486.1535.

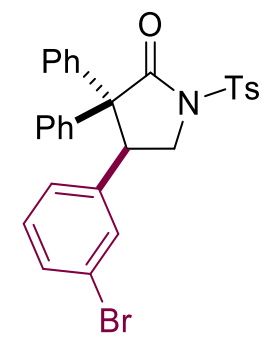

$3 g$

\section{4-(3-Bromophenyl)-3,3-diphenyl-1-tosylpyrrolidin-2-one (3g)}

Procedure A: aziridine $\mathbf{2 g}$ (77 mg, $0.219 \mathrm{mmol})$ and diphenylketene $\left(85 \mathrm{mg}, 0.438 \mathrm{mmol}\right.$ ) afforded $108 \mathrm{mg}(90 \%)$ of $\gamma^{-}$ lactam $3 \mathrm{~g}$ as a foam solid.

Procedure B: imine $1 \mathrm{~g}(78 \mathrm{mg}, 0.232 \mathrm{mmol})$ and diphenylketene $(90 \mathrm{mg}, 0.463 \mathrm{mmol})$ afforded $105 \mathrm{mg}(83 \%)$ of $\mathrm{\gamma}^{-}$ lactam $3 \mathrm{~g}$ as a foam solid.

m.p. $120-121^{\circ} \mathrm{C}$.

IR $\boldsymbol{v}_{\max }$ (neat): $3060,1734,1595,1476,1360,1166,1121,971,696 \mathrm{~cm}^{-1}$.

${ }^{1} \mathrm{H}$ NMR $\left(400 \mathrm{MHz}, \mathrm{CDCl}_{3}\right): \delta(\mathrm{ppm}) 7.93(\mathrm{~d}, J=8.5 \mathrm{~Hz}, 2 \mathrm{H}), 7.38-7.33(\mathrm{~m}, 4 \mathrm{H}), 7.29-7.25(\mathrm{~m}, 4 \mathrm{H}), 7.08(\mathrm{ps} \mathrm{t}, J=7.5 \mathrm{~Hz}$, $1 \mathrm{H}), 6.97-6.91(\mathrm{~m}, 3 \mathrm{H}), 6.76-6.73(\mathrm{~m}, 2 \mathrm{H}), 6.44(\mathrm{dd}, J=7.5,1.2 \mathrm{~Hz}, 2 \mathrm{H}), 4.40(\mathrm{ps} \mathrm{t}, J=6.8 \mathrm{~Hz}, 1 \mathrm{H}), 4.22(\mathrm{dd}, J=10.0,6.8$ $\mathrm{Hz}, 1 \mathrm{H}), 3.96(\mathrm{dd}, J=10.0,6.8 \mathrm{~Hz}, 1 \mathrm{H}), 2.48(\mathrm{~s}, 3 \mathrm{H})$.

${ }^{13} \mathrm{C} \mathrm{NMR}$ (125 MHz, $\mathrm{CDCl}_{3}$ ): $\delta$ (ppm) 173.4, 145.6, 139.4, 138.9, 138.5, 134.5, 132.1, 130.8, 129.9, 129.8, 129.3, 128.6, $128.44,128.43,128.0,127.8,127.5,127.4,122.3,64.1,49.3,46.5,21.9$.

MS (ESI) $m / z 567.7[\mathrm{M}+\mathrm{Na}]^{+}$.

HRMS (ESI) $\mathrm{m} / \mathrm{z}[\mathrm{M}+\mathrm{H}]^{+}$calcd for $\mathrm{C}_{29} \mathrm{H}_{25} \mathrm{BrNO}_{3} \mathrm{~S} 546.0733$; Found 546.0735 .

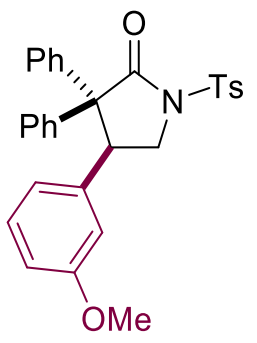

$3 \mathrm{~h}$

\section{4-(3-Methoxyphenyl)-3,3-diphenyl-1-tosylpyrrolidin-2-one (3h)}

Procedure A: aziridine $2 \mathrm{~h}(65 \mathrm{mg}, 0.214 \mathrm{mmol})$ and diphenylketene $(83 \mathrm{mg}, 0.429 \mathrm{mmol}$ ) afforded after purification by flash chromatography on silica gel $\left(\mathrm{SiO}_{2} / 2.5 \% \mathrm{Et}_{3} \mathrm{~N}\right), 82 \mathrm{mg}(77 \%)$ of $\mathrm{p}$-lactam $3 \mathrm{~h}$ as a foam solid.

Procedure B: imine $1 \mathrm{~h}(60.9 \mathrm{mg}, 0.210 \mathrm{mmol})$ and diphenylketene $(113 \mathrm{mg}, 0.421 \mathrm{mmol})$ afforded after purification by flash chromatography on silica gel $\left(\mathrm{SiO}_{2} / 2.5 \% \mathrm{Et}_{3} \mathrm{~N}\right), 91 \mathrm{mg}(87 \%)$ of $\gamma$-lactam $3 \mathrm{~h}$ as a foam solid.

m.p. $176-177^{\circ} \mathrm{C}$.

IR $\boldsymbol{v}_{\max }$ (neat): 2980, 1739, 1600, 1492, 1372, $1175 \mathrm{~cm}^{-1}$.

${ }^{1} \mathrm{H}$ NMR $\left(300 \mathrm{MHz}, \mathrm{CDCl}_{3}\right): \delta(\mathrm{ppm}) 7.91(\mathrm{~d}, J=8.5 \mathrm{~Hz}, 2 \mathrm{H}), 7.39-7.35(\mathrm{~m}, 2 \mathrm{H}), 7.32(\mathrm{~d}, J=8.0 \mathrm{~Hz}, 2 \mathrm{H}), 7.28-7.24(\mathrm{~m}$, $3 \mathrm{H}), 7.08-7.02(\mathrm{~m}, 1 \mathrm{H}), 6.99-6.91(\mathrm{~m}, 3 \mathrm{H}), 6.67(\mathrm{dd}, J=8.0,2.0 \mathrm{~Hz}, 1 \mathrm{H}), 6.53-6.50(\mathrm{~m}, 2 \mathrm{H}), 6.44(\mathrm{ps} \mathrm{d}, J=7.2 \mathrm{~Hz}, 1 \mathrm{H})$, 6.18-6.17 (m, 1H), 4.41 (ps t, $J=6.9 \mathrm{~Hz}, 1 \mathrm{H}), 4.21(\mathrm{dd}, J=9.9,6.9 \mathrm{~Hz}, 1 \mathrm{H}), 3.99(\mathrm{dd}, J=9.9,6.9 \mathrm{~Hz}, 1 \mathrm{H}), 3.53(\mathrm{~s}, 3 \mathrm{H})$, $2.47(\mathrm{~s}, 3 \mathrm{H})$. 
${ }^{13} \mathrm{C} \mathrm{NMR}\left(125 \mathrm{MHz}, \mathrm{CDCl}_{3}\right): \delta$ (ppm) 173.8, 159.3, 145.5, 139.4, 138.7, 138.5, 134.7, 129.8, 129.5, 129.3, 128.6, 128.51, $128.46,127.8,127.7,127.1,121.2,114.5,113.5,64.1,55.1,49.5,47.1,21.9$.

MS (ESI) $m / z 498.2[\mathrm{M}+\mathrm{H}]^{+}, 520.2[\mathrm{M}+\mathrm{Na}]^{+}$.

HRMS (ESI) $\mathrm{m} / \mathrm{z}[\mathrm{M}+\mathrm{H}]^{+}$calcd for $\mathrm{C}_{30} \mathrm{H}_{28} \mathrm{NO}_{4} \mathrm{~S}$ 498.1734; Found 498.1733.

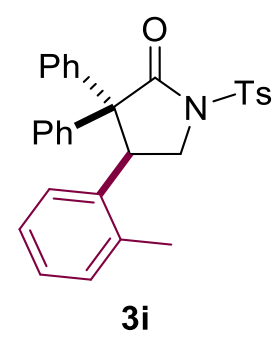

\section{3,3-Diphenyl-4-(o-tolyl)-1-tosylpyrrolidin-2-one (3i)}

Procedure A: aziridine $\mathbf{2 i}(92 \mathrm{mg}, 0.320 \mathrm{mmol})$ and diphenylketene $(124 \mathrm{mg}, 0.640 \mathrm{mmol})$ afforded $125 \mathrm{mg}(81 \%)$ of $\gamma^{-}$ lactam $3 i$ as a foam solid.

Procedure B: imine 1i $(63.3 \mathrm{mg}, 0.232 \mathrm{mmol})$ and diphenylketene $(90 \mathrm{mg}, 0.463 \mathrm{mmol})$ afforded $90 \mathrm{mg}(81 \%)$ of $\mathrm{\gamma}^{-}$ lactam $3 \mathbf{i}$ as a foam solid.

m.p. $188-190{ }^{\circ} \mathrm{C}$.

IR $\boldsymbol{v}_{\text {max }}$ (neat): $3059,2895,1733,1594,1491,1366,1170 \mathrm{~cm}^{-1}$.

${ }^{1} \mathrm{H}$ NMR $\left(400 \mathrm{MHz}, \mathrm{CDCl}_{3}\right): \delta(\mathrm{ppm}) 7.78(\mathrm{~d}, J=8.4 \mathrm{~Hz}, 2 \mathrm{H}), 7.34-7.31(\mathrm{~m}, 2 \mathrm{H}), 7.26-7.22(\mathrm{~m}, 6 \mathrm{H}), 7.03-6.98(\mathrm{~m}, 1 \mathrm{H})$, 6.97-6.94 (m, 1H), 6.92-6.87 (m, 4H), $6.67(\mathrm{ps} \mathrm{d}, J=7.2 \mathrm{~Hz}, 2 \mathrm{H}), 4.55(\mathrm{dd}, J=3.2,1.2 \mathrm{~Hz}, 1 \mathrm{H}), 4.09-4.07(\mathrm{~m}, 2 \mathrm{H}), 2.45$ (s, 3H), $2.02(\mathrm{~s}, 3 \mathrm{H})$.

${ }^{13}$ C NMR (125 MHz, CDCl $)$ ): $\delta$ (ppm) 173.9, 145.3, 138.8, 138.7, 137.8, 136.3, 134.4, 130.6, 129.8, 129.6, 129.0, 128.3, $127.9,127.7,127.4,127.1,126.8,126.7,126.5,64.2,50.9,42.6,21.9,20.2$.

MS (ESI) $m / z 482.0[\mathrm{M}+\mathrm{H}]^{+}, 503.9[\mathrm{M}+\mathrm{Na}]^{+}$.

HRMS (ESI) $\mathrm{m} / \mathrm{z}[\mathrm{M}+\mathrm{H}]^{+}$calcd for $\mathrm{C}_{30} \mathrm{H}_{28} \mathrm{NO}_{3} \mathrm{~S} 482.1784$; Found 482.1786.

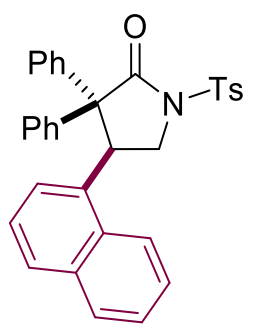

3j

4-(Naphthalen-1-yl)-3,3-diphenyl-1-tosylpyrrolidin-2-one (3j)

Procedure A: aziridine $2 \mathrm{j}(86.6 \mathrm{mg}, 0.268 \mathrm{mmol})$ and diphenylketene $(114 \mathrm{mg}, 0.589 \mathrm{mmol})$ afforded $115 \mathrm{mg}(83 \%)$ of $\mathrm{Y}$-lactam $\mathbf{3} \mathbf{j}$ as a foam solid.

Procedure B: imine $1 \mathrm{j}(71.1 \mathrm{mg}, 0.230 \mathrm{mmol})$ and diphenylketene $(89 \mathrm{mg}, 0.460 \mathrm{mmol})$ afforded $98 \mathrm{mg}(82 \%)$ of $\gamma$ lactam $3 \mathbf{j}$ as a foam solid.

m.p. $196-197^{\circ} \mathrm{C}$.

IR $v_{\max }$ (neat): $3060,2990,2958,1739,1594,1448,1359,1163 \mathrm{~cm}^{-1}$.

${ }^{1} \mathrm{H}$ NMR $\left(400 \mathrm{MHz}, \mathrm{CDCl}_{3}\right): \delta(\mathrm{ppm}) 7.85(\mathrm{~d}, J=8.8 \mathrm{~Hz}, 1 \mathrm{H}), 7.73(\mathrm{ps} \mathrm{d}, J=8.4 \mathrm{~Hz}, 2 \mathrm{H}), 7.63(\mathrm{~d}, J=8.4 \mathrm{~Hz}, 1 \mathrm{H}), 7.44-7.42$ $(\mathrm{m}, 2 \mathrm{H}), 7.40-7.37(\mathrm{~m}, 2 \mathrm{H}), 7.28-7.26(\mathrm{~m}, 5 \mathrm{H}), 7.21(\mathrm{~d}, J=8.0 \mathrm{~Hz}, 2 \mathrm{H}), 7.07(\mathrm{ps} \mathrm{t}, J=7.6 \mathrm{~Hz}, 1 \mathrm{H}), 6.98(\mathrm{~d}, J=7.2 \mathrm{~Hz}, 1 \mathrm{H})$, 6.80-6.73 (m, 4H), 5.19 (d, J = 4.4 Hz, 1H), 4.20 (dd, J =10.4, $6.0 \mathrm{~Hz}, 1 \mathrm{H}), 4.13(\mathrm{dd}, J=10.4,1.6 \mathrm{~Hz}, 1 \mathrm{H}), 2.45(\mathrm{~s}, 3 \mathrm{H})$.

${ }^{13} \mathrm{C}$ NMR (75 MHz, CDCl$)_{3}$ : $\delta$ (ppm) 173.9, 145.2, 139.5, 138.8, 134.9, 134.4, 133.8, 131.5, 129.6, 129.5, 129.1, 128.2, $128.1,127.8,127.5,127.1,126.5,126.2,125.6,125.4,125.1,122.6,63.7,51.3,42.0,21.8$.

MS (ESI) $m / z 518.0[\mathrm{M}+\mathrm{H}]^{+}, 540.0[\mathrm{M}+\mathrm{Na}]^{+}$.

HRMS (ESI) m/z [M+H] ${ }^{+}$calcd for $\mathrm{C}_{33} \mathrm{H}_{28} \mathrm{NO}_{3} \mathrm{~S}$ 518.1784; Found 518.1784. 


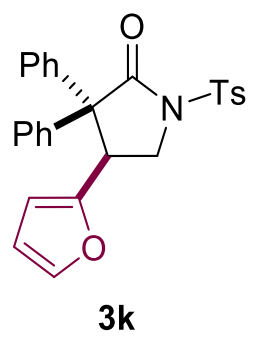

\section{4-(Furan-2-yl)-3,3-diphenyl-1-tosylpyrrolidin-2-one (3k)}

Procedure B: imine $1 \mathbf{k}(57.1 \mathrm{mg}, 0.229 \mathrm{mmol})$ and diphenylketene $(90 \mathrm{mg}, 0.463 \mathrm{mmol})$ afforded after purification by flash chromatography on silica gel $\left(\mathrm{SiO}_{2} / 2.5 \% \mathrm{Et}_{3} \mathrm{~N}\right) 16 \mathrm{mg}(15 \%)$ of $\gamma$-lactam $\mathbf{3 k}$ not pure as a slightly yellow oil.

IR $v_{\max }$ (neat): $3060,2917,1739,1597,1495,1445,1353,1153,1121,976,738 \mathrm{~cm}^{-1}$.

${ }^{1} \mathrm{H}$ NMR $\left(400 \mathrm{MHz}, \mathrm{CDCl}_{3}\right): \delta(\mathrm{ppm}) 8.10(\mathrm{~d}, J=8.2 \mathrm{~Hz}, 2 \mathrm{H}), 7.56(\mathrm{dd}, J=8.0,1.6 \mathrm{~Hz}, 2 \mathrm{H}), 7.48(\mathrm{~d}, J=8.2 \mathrm{~Hz}, 2 \mathrm{H}), 7.44-$ $7.38(\mathrm{~m}, 4 \mathrm{H}), 7.26(\mathrm{~d}, J=1.2 \mathrm{~Hz}, 1 \mathrm{H}), 7.20-7.17(\mathrm{~m}, 1 \mathrm{H}), 7.05(\mathrm{ps} \mathrm{t}, J=7.8 \mathrm{~Hz}, 2 \mathrm{H}), 6.51(\mathrm{dd}, J=8.3,1.6 \mathrm{~Hz}, 2 \mathrm{H}), 6.24$ (dd, J = 3.2, 1.6 Hz, 1H), $5.83(\mathrm{~d}, J=3.2 \mathrm{~Hz}, 1 \mathrm{H}$ ), 4.71 (pst, J = 7.6 Hz, 1H), 4.35 (dd, J = 9.6, 7.6 Hz, 1H), 4.01 (dd, J = 9.6, $8.4 \mathrm{~Hz}, 1 \mathrm{H}), 2.60(\mathrm{~s}, 3 \mathrm{H})$.

${ }^{13} \mathrm{C}$ NMR (125 MHz, CDCl $)$ : 173.3, 150.0, 145.6, 141.9, 139.4, 139.0, 134.9, 129.9, 128.9, 128.6, 128.4, 128.3, 127.8, 127.2, 110.4, 108.9, 63.4, 47.1, 40.9, 21.9.

MS (ESI) $m / z 457.9[\mathrm{M}+\mathrm{H}]^{+}, 479.9[\mathrm{M}+\mathrm{Na}]^{+}$.

HRMS (ESI) $\mathrm{m} / \mathrm{z}[\mathrm{M}+\mathrm{H}]^{+}$calcd for $\mathrm{C}_{27} \mathrm{H}_{24} \mathrm{NO}_{4} \mathrm{~S} 458.1421$; Found 458.1421.

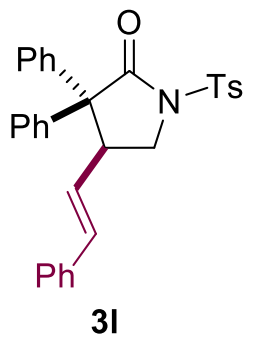

\section{3,3-Diphenyl-4-styryl-1-tosylpyrrolidin-2-one (3I)}

Procedure A: aziridine $2 \mathrm{l}(60 \mathrm{mg}, 0.200 \mathrm{mmol})$ and diphenylketene $(78 \mathrm{mg}, 0.401 \mathrm{mmol})$ afforded after purification by flash chromatography on silica gel $\left(\mathrm{SiO}_{2} / 2.5 \% \mathrm{Et}_{3} \mathrm{~N}\right), 80 \mathrm{mg}(81 \%)$ of $\gamma$-lactam $3 \mathrm{I}$ as a foam solid.

Procedure B: imine $1 \mathrm{l}(80 \mathrm{mg}, 0.280 \mathrm{mmol}$ ) and diphenylketene (109 $\mathrm{mg}, 0.561 \mathrm{mmol}$ ) afforded after purification by flash chromatography on silica gel $\left(\mathrm{SiO}_{2} / 2.5 \% \mathrm{Et}_{3} \mathrm{~N}\right), 122 \mathrm{mg}(88 \%)$ of $\gamma$-lactam $3 \mathrm{l}$ as a foam solid.

m.p. $83-84^{\circ} \mathrm{C}$.

IR $v_{\max }$ (neat): $3058,3025,1734,1596,1445,1362,1169,1087 \mathrm{~cm}^{-1}$.

${ }^{1} \mathrm{H}$ NMR $\left(400 \mathrm{MHz}, \mathrm{CDCl}_{3}\right): \delta(\mathrm{ppm}) 8.01(\mathrm{~d}, J=8.4 \mathrm{~Hz}, 2 \mathrm{H}), 7.40-7.37(\mathrm{~m}, 4 \mathrm{H}), 7.30-7.22(\mathrm{~m}, 7 \mathrm{H}), 7.18-7.16(\mathrm{~m}, 2 \mathrm{H})$, 7.10 (ps t $J=8.0 \mathrm{~Hz}, 2 \mathrm{H}), 6.64-6.59(\mathrm{~m}, 3 \mathrm{H}), 5.64(\mathrm{dd}, J=16.0,8.8 \mathrm{~Hz}, 1 \mathrm{H}), 4.15$ (dd, $J=9.6,7.2 \mathrm{~Hz}, 1 \mathrm{H}), 4.03(\mathrm{dd}, J=$ $16.0,8.4 \mathrm{~Hz}, 1 \mathrm{H}$ ), 3.56 (ps t, $J=9.6 \mathrm{~Hz}, 1 \mathrm{H}), 2.49(\mathrm{~s}, 3 \mathrm{H}$ ).

${ }^{13} \mathrm{C}$ NMR (125 MHz, CDCl $)$ : 174.1, 145.6, 139.8, 139.9, 136.5, 135.0, 134.1, 129.9, 128.9, 128.8, 128.7, 128.6, 128.3, $128.22,128.15,127.7,127.6,126.4,125.2,62.3,48.6,44.6,21.9$.

MS (ESI) $m / z 493.9[\mathrm{M}+\mathrm{H}]^{+}, 515.9[\mathrm{M}+\mathrm{Na}]^{+}$.

HRMS (ESI) $\mathrm{m} / \mathrm{z}[\mathrm{M}+\mathrm{H}]^{+}$calcd for $\mathrm{C}_{31} \mathrm{H}_{28} \mathrm{NO}_{3} \mathrm{~S}$ 494.1784; Found 494.1786.

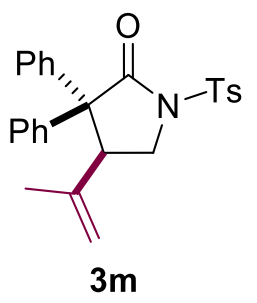

3,3-Diphenyl-4-(prop-1-en-2-yl)-1-tosylpyrrolidin-2-one (144n) 
Procedure A: aziridine $2 \mathrm{~m}(17.8 \mathrm{mg}, 0.075 \mathrm{mmol})$ and diphenylketene $(29.1 \mathrm{mg}, 0.150 \mathrm{mmol})$ afforded $14 \mathrm{mg}(43 \%)$ of $\gamma$-lactam $3 \mathrm{~m}$ as a colourless oil.

IR $\boldsymbol{V}_{\max }\left(\right.$ neat): $3059,2921,1735,1646,1596,1494,1364,1170 \mathrm{~cm}^{-1}$.

${ }^{1} \mathrm{H}$ NMR $\left(400 \mathrm{MHz}, \mathrm{CDCl}_{3}\right): \delta(\mathrm{ppm}) 7.83(\mathrm{~d}, J=8.4 \mathrm{~Hz}, 2 \mathrm{H}), 7.26-7.20(\mathrm{~m}, 7 \mathrm{H}), 7.16-7.08(\mathrm{~m}, 3 \mathrm{H}), 7.01(\mathrm{~d}, J=7.2 \mathrm{~Hz}$, $2 \mathrm{H}), 4.78(\mathrm{~s}, 1 \mathrm{H}), 4.69(\mathrm{~s}, 1 \mathrm{H}), 3.99(\mathrm{dd}, J=11.6,8.0 \mathrm{~Hz}, 1 \mathrm{H}), 3.82-3.78(\mathrm{~m}, 2 \mathrm{H}), 2.44(\mathrm{~s}, 3 \mathrm{H}), 1.35(\mathrm{~s}, 3 \mathrm{H})$.

${ }^{13} \mathrm{C} \mathrm{NMR}\left(75 \mathrm{MHz}, \mathrm{CDCl}_{3}\right): \delta$ (ppm) 173.6, 145.3, 142.5, 139.6, 138.7, 134.7, 129.7, 129.2, 128.6, 128.3, 128.2, 127.9, 127.7, 127.1, 115.8, 62.4, 48.4, 48.2, 22.5, 21.8 .

MS (ESI) $m / z 432.0[\mathrm{M}+\mathrm{H}]^{+}, 454.0[\mathrm{M}+\mathrm{Na}]^{+}$.

HRMS (ESI) $\mathrm{m} / \mathrm{z}[\mathrm{M}+\mathrm{H}]^{+}$calcd for $\mathrm{C}_{26} \mathrm{H}_{26} \mathrm{NO}_{3} \mathrm{~S}$ 432.1628; Found 432.1628

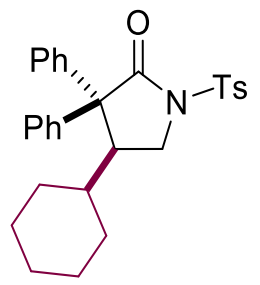

$3 n$

\section{4-Cyclohexyl-3,3-diphenyl-1-tosylpyrrolidin-2-one (144m)}

Procedure A: aziridine $2 \mathrm{n}(65 \mathrm{mg}, 0.233 \mathrm{mmol})$ and diphenylketene $(181 \mathrm{mg}, 0.931 \mathrm{mmol}$ ) afforded $53 \mathrm{mg}(48 \%)$ of not pure $\boldsymbol{\gamma}$-lactam $3 n$ as a colourless oil.

Procedure B: imine 1n (60 mg, $0.226 \mathrm{mmol})$ and diphenylketene $(88 \mathrm{mg}, 0.452 \mathrm{mmol}$ ) afforded $73 \mathrm{mg}(68 \%)$ of not pure $\boldsymbol{\gamma}$-lactam $3 \mathrm{n}$ as a colourless oil.

IR $\boldsymbol{v}_{\max }$ (neat): $3056,2908,2850,1724,1651,1598,1494,1444,1364,1141 \mathrm{~cm}^{-1}$.

${ }^{1} \mathrm{H}$ NMR $\left(500 \mathrm{MHz}, \mathrm{CDCl}_{3}\right): \delta(\mathrm{ppm}) 7.54(\mathrm{~d}, J=8.0 \mathrm{~Hz}, 2 \mathrm{H}), 7.31-7.23(\mathrm{~m}, 11 \mathrm{H}), 7.20-7.17(\mathrm{~m}, 1 \mathrm{H}), 3.92(\mathrm{ps} \mathrm{t}, J=7.5 \mathrm{~Hz}$, $2 \mathrm{H}$ ), 3.39 (dd, $J=8.3,7.0 \mathrm{~Hz}, 1 \mathrm{H}$ ), $2.45(\mathrm{~s}, 3 \mathrm{H}), 1.99(\mathrm{~d}, J=13.5,1 \mathrm{H}$ ), 1.79 (ps t, $J=13.5 \mathrm{~Hz}, 2 \mathrm{H}$ ), 1.69 (ps t, $J=15.0 \mathrm{~Hz}$, $2 \mathrm{H}), 1.29-1.17(\mathrm{~m}, 4 \mathrm{H}), 1.02-0.93(\mathrm{~m}, 2 \mathrm{H})$.

${ }^{13} \mathrm{C}$ NMR (125 MHz, CDCl $): \delta$ (ppm) 145.1, 144.6, 140.9, 140.0, 135.3, 131.0, 130.0, 129.8, 127.99, 127.97, 127.95, 126.6, 126.4, 111.1, 67.1, 64.8, 39.8, 30.0, 29.5, 26.5, 25.9, 25.8, 21.8.

MS (ESI) $\mathrm{m} / \mathrm{z} 474.0[\mathrm{M}+\mathrm{H}]^{+}, 496.1[\mathrm{M}+\mathrm{Na}]^{+}$.

HRMS (ESI) $\mathrm{m} / \mathrm{z}[\mathrm{M}+\mathrm{H}]^{+}$calcd for $\mathrm{C}_{29} \mathrm{H}_{32} \mathrm{NO}_{3} \mathrm{~S}$ 474.2097; Found 474.2099.

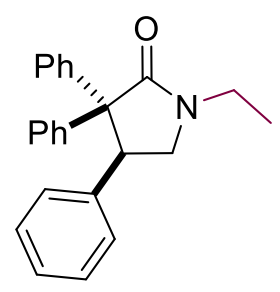

30

\section{1-Ethyl-3,3,4-triphenylpyrrolidin-2-one (30)}

Following the Procedure A of $\mathrm{Y}$-lactam synthesis: to a stirred solution of $\mathrm{N}$-ethylaziridine $20(50.0 \mathrm{mg}, 0.340 \mathrm{mmol}, 1$ eq) and lithium iodide $(36.4 \mathrm{mg}, 0.272 \mathrm{mmol}, 0.8 \mathrm{eq})$ in dry THF $(1.5 \mathrm{~mL})$ was added via syringe diphenylketene (132 $\mathrm{mg}, 0.679 \mathrm{mmol}, 2 \mathrm{eq})$ in dry THF (1.5 mL) over 1 hour, under argon. After being stirred 2 hours at room temperature, the solution was quenched with saturated aqueous $\mathrm{NaHCO}_{3}$ solution, and the product was extracted three times with EtOAc. The organic layers were washed with saturated aqueous $\mathrm{NaCl}$ solution and dried over $\mathrm{Na}_{2} \mathrm{SO}_{4}$, filtered and concentrated in vacuo. Flash chromatography on silica gel (eluent EtOAc/Pentane, 10:90 to 30:70) of the crude mixture gave $70 \mathrm{mg}(60 \%)$ of $\mathrm{p}$-lactam 30 as a white solid.

m.p. $160-161^{\circ} \mathrm{C}$.

IR $\boldsymbol{v}_{\max }$ (neat): $3057,2975,2932,1741,1682,1595,1486,1443,1285,734 \mathrm{~cm}^{-1}$.

${ }^{1} \mathrm{H}$ NMR $\left(500 \mathrm{MHz}, \mathrm{CDCl}_{3}\right): \delta(\mathrm{ppm}) 7.61(\mathrm{~d}, J=7.8 \mathrm{~Hz}, 2 \mathrm{H}), 7.35$ (ps t, $\left.J=7.8 \mathrm{~Hz}, 2 \mathrm{H}\right), 7.29-7.27(\mathrm{~m}, 1 \mathrm{H}), 7.11-6.98(\mathrm{~m}$, $6 \mathrm{H}), 6.81(\mathrm{~d}, J=7.0 \mathrm{~Hz}, 2 \mathrm{H}), 6.76(\mathrm{~d}, J=7.0 \mathrm{~Hz}, 2 \mathrm{H}), 4.48$ (ps t, $J=7.0 \mathrm{~Hz}, 1 \mathrm{H}, \mathrm{CH}), 3.72(\mathrm{dd}, J=9.8,7.0 \mathrm{~Hz}, 1 \mathrm{H}), 3.69-$ $3.57(\mathrm{~m}, 2 \mathrm{H}), 3.53(\mathrm{dq}, J=10.4,7.0 \mathrm{~Hz}, 1 \mathrm{H}), 1.25(\mathrm{t}, J=7.3 \mathrm{~Hz}, 3 \mathrm{H})$. 
$\left.{ }^{13} \mathrm{C} \mathrm{NMR} \mathrm{(125} \mathrm{MHz,} \mathrm{CDCl} 3\right): \delta$ (ppm) 174.4, 142.0, 140.5, 138.9, 129.9, 129.2, 128.8, 128.2, 128.0, 127.5, 127.2, 127.0, $126.5,63.5,49.5,48.0,37.9,12.3$.

MS (ESI) $m / z 364.0[\mathrm{M}+\mathrm{Na}]^{+}$.

HRMS (ESI) $\mathrm{m} / \mathrm{z}[\mathrm{M}+\mathrm{H}]^{+}$calcd for $\mathrm{C}_{24} \mathrm{H}_{24} \mathrm{NO} 342.1852$; Found 342.1853.

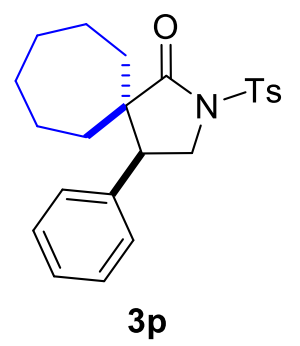

\section{4-Phenyl-2-tosyl-2-azaspiro[4.6]undecan-1-one (3p)}

Procedure B: imine 1a $(50.0 \mathrm{mg}, 0.193 \mathrm{mmol})$ and cycloheptylketene $(48.0 \mathrm{mg}, 0.386 \mathrm{mmol})$ afforded $61.0 \mathrm{mg}(80 \%)$ of $\gamma$-lactam $3 p$ as a foam solid.

m.p. $51-52{ }^{\circ} \mathrm{C}$.

IR $v_{\max }$ (neat): 2923, 2854, 1726, 1596, 1494, 1456, 1353, 1166, $1119 \mathrm{~cm}^{-1}$.

${ }^{1} \mathrm{H}$ NMR $\left(400 \mathrm{MHz}, \mathrm{CDCl}_{3}\right): \delta(\mathrm{ppm}) 7.97(\mathrm{~d}, J=8.4 \mathrm{~Hz}, 2 \mathrm{H}), 7.37(\mathrm{~d}, J=8.4 \mathrm{~Hz}, 2 \mathrm{H}), 7.31-7.22(\mathrm{~m}, 3 \mathrm{H}), 7.06-7.03(\mathrm{~m}$, $2 \mathrm{H}$ ), $4.12(\mathrm{dd}, J=10.4,7.2 \mathrm{~Hz}, 1 \mathrm{H}), 3.97(\mathrm{dd}, J=10.4,6.4 \mathrm{~Hz}, 1 \mathrm{H}$ ), 3.19 (ps t, $J=6.4 \mathrm{~Hz}, 1 \mathrm{H}), 2.48(\mathrm{~s}, 3 \mathrm{H}), 1.94-1.86(\mathrm{~m}$, $1 \mathrm{H}), 1.72-1.66(\mathrm{~m}, 3 \mathrm{H}), 1.50-1.31(\mathrm{~m}, 5 \mathrm{H}), 1.27-1.20(\mathrm{~m}, 2 \mathrm{H}), 0.99-0.86(\mathrm{~m}, 1 \mathrm{H})$.

${ }^{13} \mathrm{C}$ NMR (125 MHz, CDCl $)$ : $\delta$ (ppm) 178.4, 145.3, 138.1, 135.4, 129.9, 128.8, 128.3, 128.2, 127.8, 52.5, 49.6, 49.5, 36.5, 30.82, 30.80, 30.75, 23.4, 22.8, 21.9.

MS (APCI) $m / z 398.2[\mathrm{M}+\mathrm{H}]^{+}$.

HRMS (ESI) $\mathrm{m} / \mathrm{z}[\mathrm{M}+\mathrm{H}]^{+}$calcd for $\mathrm{C}_{23} \mathrm{H}_{28} \mathrm{NO}_{3} \mathrm{~S} 398.1784$; Found 398.1787 .

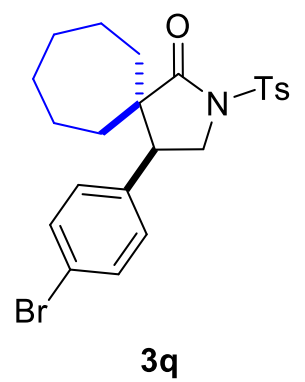

\section{4-(4-Bromophenyl)-2-tosyl-2-azaspiro[4.6]undecan-1-one (3q)}

Procedure B: imine 1c $(85.0 \mathrm{mg}, 0.250 \mathrm{mmol})$ and cycloheptylketene $(62.1 \mathrm{mg}, 0.500 \mathrm{mmol})$ afforded $92.0 \mathrm{mg}(77 \%)$ of $\gamma$-lactam $3 q$ as a foam solid.

m.p. $61-62{ }^{\circ} \mathrm{C}$.

IR $v_{\max }$ (neat): 2923, 2854, 1731, 1596, 1490, 1460, 1355, 1166, $1116 \mathrm{~cm}^{-1}$.

${ }^{1} \mathrm{H}$ NMR $\left(500 \mathrm{MHz}, \mathrm{CDCl}_{3}\right): \delta(\mathrm{ppm}) 7.95(\mathrm{~d}, J=8.5 \mathrm{~Hz}, 2 \mathrm{H}), 7.37(\mathrm{~d}, J=8.5 \mathrm{~Hz}, 4 \mathrm{H}), 6.91(\mathrm{~d}, J=8.5 \mathrm{~Hz}, 2 \mathrm{H}), 4.10(\mathrm{dd}, J=$ 10.5, 7.0 Hz, 1H), 3.90 (dd, $J=10.5,6.0 \mathrm{~Hz}, 1 \mathrm{H}$ ), 3.14 (ps t, $J=6.5 \mathrm{~Hz}, 1 \mathrm{H}), 2.48(\mathrm{~s}, 3 \mathrm{H}), 1.89$ (dd, $J=13.8,9.5 \mathrm{~Hz}, 1 \mathrm{H}$ ), $1.73-1.62(\mathrm{~m}, 2 \mathrm{H}), 1.50-1.31(\mathrm{~m}, 7 \mathrm{H}), 1.20-1.16(\mathrm{~m}, 1 \mathrm{H}), 0.99-0.93(\mathrm{~m}, 1 \mathrm{H})$.

${ }^{13} \mathrm{C}$ NMR (125 MHz, CDCl $): \delta$ (ppm) 178.0, 145.4, 137.3, 135.2, 131.9, 129.89, 129.86, 128.2, 121.7, 52.4, 49.3, 49.0, 36.5, 30.76, 30.74, 30.72, 23.4, 22.8, 21.9.

MS (ESI) $m / z 475.9[\mathrm{M}+\mathrm{H}]^{+} .497 .7[\mathrm{M}+\mathrm{Na}]^{+}$.

HRMS (ESI) $\mathrm{m} / \mathrm{z}[\mathrm{M}+\mathrm{H}]^{+}$calcd for $\mathrm{C}_{23} \mathrm{H}_{27} \mathrm{BrNO}_{3} \mathrm{~S}$ 476.0890; Found 476.0890 . 


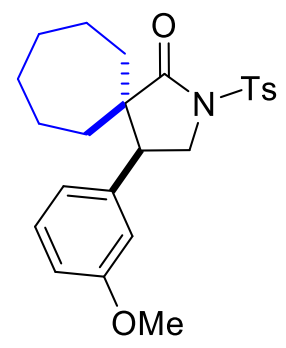

$3 r$

4-(3-Methoxyphenyl)-2-tosyl-2-azaspiro[4.6]undecan-1-one (3r)

Procedure B: imine $1 \mathrm{~h}(72.3 \mathrm{mg}, 0.250 \mathrm{mmol})$ and cycloheptylketene $(62.1 \mathrm{mg}, 0.500 \mathrm{mmol})$ afforded after purification by flash chromatography on silica gel $\left(\mathrm{SiO}_{2} / 2.5 \% \mathrm{Et}_{3} \mathrm{~N}\right) 94.0 \mathrm{mg}(88 \%)$ of $\gamma$-lactam $3 \mathrm{r}$ as a foam solid.

m.p. $49-50^{\circ} \mathrm{C}$.

IR $\boldsymbol{v}_{\max }$ (neat): 2921, 2853, 1731, 1598, 1491, 1455, 1360, 1167, $1115 \mathrm{~cm}^{-1}$.

${ }^{1} \mathrm{H}$ NMR $\left(400 \mathrm{MHz}, \mathrm{CDCl}_{3}\right): \delta(\mathrm{ppm}) 7.96(\mathrm{~d}, J=8.4 \mathrm{~Hz}, 2 \mathrm{H}), 7.35(\mathrm{~d}, J=8.4 \mathrm{~Hz}, 2 \mathrm{H}), 7.15$ (ps t, $\left.J=9.5 \mathrm{~Hz}, 1 \mathrm{H}\right), 6.80-6.78$ (m, $1 \mathrm{H}), 6.62-6.60(\mathrm{~m}, 2 \mathrm{H}), 4.10(\mathrm{dd}, J=10.4,7.2 \mathrm{~Hz}, 1 \mathrm{H}), 3.95(\mathrm{dd}, J=10.4,6.0 \mathrm{~Hz}, 1 \mathrm{H}), 3.74(\mathrm{~s}, 3 \mathrm{H}), 3.14(\mathrm{ps} t, J=6.8$ $\mathrm{Hz}, 1 \mathrm{H}), 2.46(\mathrm{~s}, 3 \mathrm{H}), 1.94-1.84(\mathrm{~m}, 1 \mathrm{H}), 1.74-1.65(\mathrm{~m}, 2 \mathrm{H}), 1.31-1.50(\mathrm{~m}, 6 \mathrm{H}), 1.31-1.22(\mathrm{~m}, 2 \mathrm{H}), 1.02-0.95(\mathrm{~m}, 1 \mathrm{H})$.

${ }^{13}$ C NMR (75 MHz, CDCl $): \delta$ (ppm) 178.3, 159.8, 145.2, 139.8, 135.4, 129.8, 129.7, 128.2, 120.5, 114.5, 112.7, 55.3, $52.5,49.52,49.45,36.6,30.78,30.75,30.71,23.4,22.8,21.9$.

MS (ESI) $m / z 428.1[\mathrm{M}+\mathrm{H}]^{+}$.

HRMS (ESI) $\mathrm{m} / \mathrm{z}[\mathrm{M}+\mathrm{H}]^{+}$calcd for $\mathrm{C}_{24} \mathrm{H}_{30} \mathrm{NO}_{4} \mathrm{~S} 428.1890$; Found 428.1893.

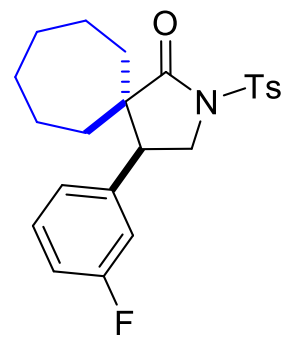

3s

\section{4-(3-Fluorophenyl)-2-tosyl-2-azaspiro[4.6]undecan-1-one (181f)}

Procedure B: imine 1f $(69.3 \mathrm{mg}, 0.250 \mathrm{mmol})$ and cycloheptylketene $(62.1 \mathrm{mg}, 0.500 \mathrm{mmol})$ afforded $84.0 \mathrm{mg}(81 \%)$ of $\gamma$-lactam $3 \mathrm{~s}$ as a foam solid.

m.p. $48-49^{\circ} \mathrm{C}$.

IR $v_{\max }$ (neat): 2924, 2854, 1732, 1589, 1489, 1449, 1359, 1170, $1117 \mathrm{~cm}^{-1}$.

${ }^{1} \mathrm{H}$ NMR $\left(500 \mathrm{MHz}, \mathrm{CDCl}_{3}\right): \delta(\mathrm{ppm}) 7.95(\mathrm{~d}, J=8.0 \mathrm{~Hz}, 2 \mathrm{H}), 7.37(\mathrm{~d}, J=8.0 \mathrm{~Hz}, 2 \mathrm{H}), 7.23-7.18(\mathrm{~m}, 1 \mathrm{H}), 6.94(\mathrm{td}, J=8.0$, $2.0 \mathrm{~Hz}, 1 \mathrm{H}), 6.82(\mathrm{~d}, J=7.5 \mathrm{~Hz}, 1 \mathrm{H}), 6.67-6.64(\mathrm{~m}, 1 \mathrm{H}), 4.12(\mathrm{dd}, J=10.5,7.5 \mathrm{~Hz}, 1 \mathrm{H}), 3.91(\mathrm{dd}, J=10.5,5.5 \mathrm{~Hz}, 1 \mathrm{H}), 3.17$ (ps t, J = 6.5 Hz, 1H), $2.47(\mathrm{~s}, 3 \mathrm{H}), 1.94-1.88(\mathrm{~m}, 1 \mathrm{H}), 1.74-1.65(\mathrm{~m}, 2 \mathrm{H}), 1.45-1.16(\mathrm{~m}, 8 \mathrm{H}), 1.00-0.95(\mathrm{~m}, 1 \mathrm{H})$.

${ }^{13} \mathrm{C}$ NMR (125 MHz, CDCl $): \delta(\mathrm{ppm})$ 178.0, $163.8(\mathrm{C}-\mathrm{F}, 1 \mathrm{JC}-\mathrm{F}=246.0 \mathrm{~Hz}), 161.9(\mathrm{C}-\mathrm{F}, 1 \mathrm{JC}-\mathrm{F}=246.0 \mathrm{~Hz}), 145.5,140.91$ $(C-F, 3 J C-F=6.3 \mathrm{~Hz}), 140.85(C-F, 3 J C-F=6.3 \mathrm{~Hz}), 135.1,130.3(C-F, 3 J C-F=8.7 \mathrm{~Hz}), 130.2(C-F, 3 J C-F=8.7 \mathrm{~Hz}), 129.9$, $128.1,123.95(C-F, 4 J C-F=2.5 \mathrm{~Hz}), 123.92(C-F, 4 J C-F=2.5 \mathrm{~Hz}), 115.1(C-F, 2 J C-F=21.3 \mathrm{~Hz}), 114.9(C-F, 2 J C-F=21.3$ $\mathrm{Hz}), 114.8(\mathrm{C}-\mathrm{F}, 2 \mathrm{JC}-\mathrm{F}=21.3 \mathrm{~Hz}), 114.6(\mathrm{C}-\mathrm{F}, 2 \mathrm{JC}-\mathrm{F}=21.3 \mathrm{~Hz}), 21.3 \mathrm{~Hz}), 52.5,49.4,49.2,36.5,30.8,23.4,22.8,21.9$.

${ }^{19} \mathrm{~F}$ NMR (376 MHz, $\left.\mathrm{CDCl}_{3}\right): \delta(\mathrm{ppm})-111.4$

MS (ESI) $m / z 416.0[\mathrm{M}+\mathrm{H}]^{+}, 437.9[\mathrm{M}+\mathrm{Na}]^{+}$.

HRMS (ESI) $\mathrm{m} / \mathrm{z}[\mathrm{M}+\mathrm{H}]^{+}$calcd for $\mathrm{C}_{23} \mathrm{H}_{27} \mathrm{FNO}_{3} \mathrm{~S}$ 416.1690; Found 416.1692. 


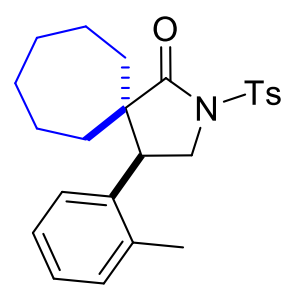

$3 \mathbf{t}$

\section{4-(o-Tolyl)-2-tosyl-2-azaspiro[4.6]undecan-1-one (181i)}

Procedure B: imine 1i $(68.3 \mathrm{mg}, 0.250 \mathrm{mmol})$ and cycloheptylketene $(62.1 \mathrm{mg}, 0.500 \mathrm{mmol})$ afforded $83.0 \mathrm{mg}(81 \%)$ of $Y$-lactam $3 t$ as a colourless oil.

IR $v_{\max }$ (neat): 2923, 2854, 1730, 1596, 1492, 1462, 1358, 1169, $1117 \mathrm{~cm}^{-1}$.

${ }^{1} \mathrm{H}$ NMR $\left(500 \mathrm{MHz}, \mathrm{CDCl}_{3}\right): \delta(\mathrm{ppm}) 7.97(\mathrm{~d}, J=8.0 \mathrm{~Hz}, 2 \mathrm{H}), 7.37(\mathrm{~d}, J=8.0 \mathrm{~Hz}, 2 \mathrm{H}), 7.14-7.08(\mathrm{~m}, 2 \mathrm{H}), 6.92(\mathrm{ps} \mathrm{t}, J=8.0$ $\mathrm{Hz}, 1 \mathrm{H}), 6.72(\mathrm{~d}, J=8.0 \mathrm{~Hz}, 1 \mathrm{H}), 4.16(\mathrm{dd}, J=10.5,7.5 \mathrm{~Hz}, 1 \mathrm{H}), 3.87(\mathrm{dd}, J=10.5,3.5 \mathrm{~Hz}, 1 \mathrm{H}), 3.50(\mathrm{dd}, J=7.5,3.5 \mathrm{~Hz}$, $1 \mathrm{H}), 2.48(\mathrm{~s}, 3 \mathrm{H}), 2.33(\mathrm{~s}, 3 \mathrm{H}), 1.87-1.84(\mathrm{~m}, 1 \mathrm{H}), 1.79-1.68(\mathrm{~m}, 2 \mathrm{H}), 1.56-1.26(\mathrm{~m}, 8 \mathrm{H}), 1.07-1.02(\mathrm{~m}, 1 \mathrm{H})$.

$\left.{ }^{13} \mathrm{C} \mathrm{NMR} \mathrm{(125} \mathrm{MHz,} \mathrm{CDCl}_{3}\right): \delta$ (ppm) 178.7, 145.3, 138.2, 136.2, 135.3, 130.9, 129.8, 128.2, 127.2, 126.6, 126.3, 53.2, 50.9, 43.1, 36.6, 31.6, 31.2, 30.8, 23.7, 23.1, 21.9, 20.5.

MS (ESI) $m / z 411.9[\mathrm{M}+\mathrm{H}]^{+}, 433.9[\mathrm{M}+\mathrm{Na}]^{+}$.

HRMS (ESI) $\mathrm{m} / \mathrm{z}[\mathrm{M}+\mathrm{H}]^{+}$calcd for $\mathrm{C}_{24} \mathrm{H}_{30} \mathrm{NO}_{3} \mathrm{~S}$ 412.1941; Found 412.1944.

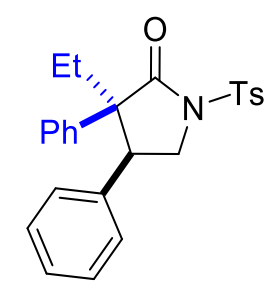

$3 \mathbf{u}$

\section{3-Ethyl-3,4-diphenyl-1-tosylpyrrolidin-2-one (3u)}

Procedure B: imine 1a $(66.0 \mathrm{mg}, 0.255 \mathrm{mmol})$ and ethylphenylketene $(74.4 \mathrm{mg}, 0.509 \mathrm{mmol})$ afforded $92.0 \mathrm{mg}(86 \%)$ of $\mathrm{p}$-lactam $3 \mathbf{u}$ as a foam solid.

m.p. $141-142^{\circ} \mathrm{C}$.

IR $\boldsymbol{V}_{\max }$ (neat): $3035,2995,1733,1596,1498,1363,1176,1088 \mathrm{~cm}^{-1}$.

${ }^{1} \mathrm{H} \mathrm{NMR}\left(500 \mathrm{MHz}, \mathrm{CDCl}_{3}\right): \delta(\mathrm{ppm}) 8.09(\mathrm{~d}, J=8.0 \mathrm{~Hz}, 2 \mathrm{H}), 7.45(\mathrm{~d}, J=8.0 \mathrm{~Hz}, 2 \mathrm{H}), 7.18$ (ps t, $\left.J=7.0 \mathrm{~Hz}, 1 \mathrm{H}\right), 7.10$ (ps t, $J=8.0 \mathrm{~Hz}, 2 \mathrm{H}), 7.05($ ps t, $J=7.0 \mathrm{~Hz}, 1 \mathrm{H}), 6.88($ ps t, $J=8.0 \mathrm{~Hz}, 2 \mathrm{H}), 6.61(\mathrm{~d}, J=7.5 \mathrm{~Hz}, 2 \mathrm{H}), 6.21(\mathrm{~d}, J=8.0 \mathrm{~Hz}, 2 \mathrm{H}), 4.16$ (dd, $J=10.0,7.5 \mathrm{~Hz}, 1 \mathrm{H}$ ), 3.80 (ps t, $J=10.0 \mathrm{~Hz}, 1 \mathrm{H}$ ), $3.65(\mathrm{dd}, J=10.0,7.5 \mathrm{~Hz}, 1 \mathrm{H}) 2.53(\mathrm{~s}, 3 \mathrm{H}), 2.03-1.95(\mathrm{~m}, 2 \mathrm{H}), 0.98$ $(\mathrm{t}, J=7.5 \mathrm{~Hz}, 3 \mathrm{H})$.

$\left.{ }^{13} \mathrm{C} \mathrm{NMR} \mathrm{(125} \mathrm{MHz,} \mathrm{CDCl} 3\right): \delta$ (ppm) 175.7, 145.6, 137.0, 135.43, 135.35, 130.0, 129.0, 128.6, 128.2, 127.9, 127.8, 127.5, 127.1, 59.3, 48.3, 47.8, 28.1, 21.9, 9.1.

MS (ESI) $m / z 419.8[\mathrm{M}+\mathrm{H}]^{+}, 441.8[\mathrm{M}+\mathrm{Na}]^{+}$.

HRMS (ESI) $\mathrm{m} / \mathrm{z}[\mathrm{M}+\mathrm{H}]^{+}$calcd for $\mathrm{C}_{25} \mathrm{H}_{26} \mathrm{NO}_{3} \mathrm{~S}$ 420.1628; Found 420.1630.

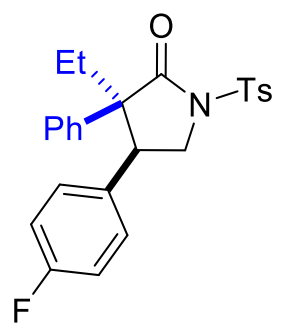

3v

\section{3-Ethyl-4-(4-fluorophenyl)-3-phenyl-1-tosylpyrrolidin-2-one (3v)}

Procedure B: imine $\mathbf{1 b}(71.3 \mathrm{mg}, 0.257 \mathrm{mmol})$ and ethylphenylketene $(75.0 \mathrm{mg}, 0.515 \mathrm{mmol})$ afforded $92.0 \mathrm{mg}(82 \%)$ of $\gamma$-lactam $3 \mathbf{v}$ as a white solid. 
m.p. $138-140{ }^{\circ} \mathrm{C}$.

IR $\boldsymbol{v}_{\max }$ (neat): 3032, 2990, 1734, 1511, 1368, 1224, 1186, $1170 \mathrm{~cm}^{-1}$.

${ }^{1} \mathrm{H}$ NMR (500 MHz, CDCl 3$): \delta(p p m) 8.08(\mathrm{~d}, J=8.5 \mathrm{~Hz}, 2 \mathrm{H}), 7.45(\mathrm{~d}, J=8.5 \mathrm{~Hz}, 2 \mathrm{H}),(\mathrm{ps} \mathrm{t}, J=7.5 \mathrm{~Hz}, 1 \mathrm{H}), 6.91(\mathrm{pst}, J=$ $8.5 \mathrm{~Hz}, 2 \mathrm{H}), 6.79$ (ps t, $J=8.5 \mathrm{~Hz}, 2 \mathrm{H}), 6.56(\mathrm{dd}, J=8.8,5.5 \mathrm{~Hz}, 2 \mathrm{H}), 6.22(\mathrm{~d}, J=7.5 \mathrm{~Hz}, 2 \mathrm{H}), 4.15(\mathrm{dd}, J=10.0,7.0 \mathrm{~Hz}$, $1 \mathrm{H}), 3.74$ (ps t, $J=10.0 \mathrm{~Hz}, 1 \mathrm{H}), 3.63(\mathrm{dd}, J=10.0,7.0 \mathrm{~Hz}, 1 \mathrm{H}), 2.52(\mathrm{~s}, 3 \mathrm{H}), 12.04-1.90(\mathrm{~m}, 2 \mathrm{H}), 0.96(\mathrm{t}, J=7.5 \mathrm{~Hz}, 3 \mathrm{H})$. ${ }^{13} \mathrm{C}$ NMR (125 MHz, CDCl$): \delta(p p m) 175.5, c 163.3(\mathrm{C}-\mathrm{F}, 1 \mathrm{JC}-\mathrm{F}=247 \mathrm{~Hz}), 161.3(\mathrm{C}-\mathrm{F}, 1 \mathrm{JC}-\mathrm{F}=247 \mathrm{~Hz}), 145.7,136.9,135.3$, 131.21 (C-F, 4JC-F = 3.8 Hz), 131.18 (C-F, 4JC-F = 3.8 Hz), 130.53 (C-F, 3JC-F = 7.5 Hz), 130.46 (C-F, 3JC-F = 7.5 Hz), 130.0, 128.5, 128.1, 127.5, 127.3, 115.2 (C-F, 2JC-F = 21.3 Hz), $115.0(C-F, 2 J C-F=21.3 \mathrm{~Hz}), 59.2,48.4,47.2,28.0,21.9,9.0$.

$\left.{ }^{19} \mathrm{~F} \mathrm{NMR} \mathrm{(376} \mathrm{MHz,} \mathrm{CDCl}_{3}\right): \delta(\mathrm{ppm})-114.0$

MS (ESI) $m / z 438.0[\mathrm{M}+\mathrm{H}]^{+}$.

HRMS (ESI) m/z [M+H] ${ }^{+}$calcd for $\mathrm{C}_{25} \mathrm{H}_{25} \mathrm{FNO}_{3} \mathrm{~S}$ 438.1534; Found 438.1534.

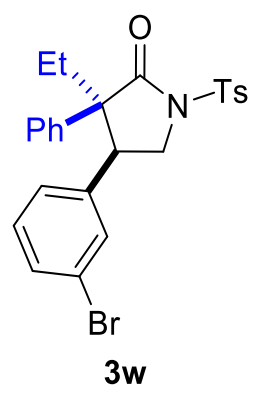

\section{4-(3-Bromophenyl)-3-ethyl-3-phenyl-1-tosylpyrrolidin-2-one (3w)}

Procedure B: imine $1 \mathrm{~g}(87 \mathrm{mg}, 0.257 \mathrm{mmol})$ and ethylphenylketene (75 mg, $0.514 \mathrm{mmol})$ afforded $95 \mathrm{mg}$ (75\%) of $\gamma$ lactam $3 \mathbf{w}$ as a foam solid

m.p. $177-178^{\circ} \mathrm{C}$.

IR $\boldsymbol{v}_{\max }$ (neat): 3067, 2972, 2938, 1737, 1595, 1367, 1172, $1110 \mathrm{~cm}^{-1}$.

${ }^{1} \mathrm{H}$ NMR (500 MHz, CDCl 3$): \delta(p p m) 8.08(\mathrm{~d}, J=8.5 \mathrm{~Hz}, 2 \mathrm{H}), 7.46(\mathrm{~d}, J=8.5 \mathrm{~Hz}, 2 \mathrm{H}), 7.30(\mathrm{~d}, J=8.0 \mathrm{~Hz}, 1 \mathrm{H}), 7.09(\mathrm{pst}, J$ $=7.5 \mathrm{~Hz}, 1 \mathrm{H}), 6.98-6.92(\mathrm{~m}, 3 \mathrm{H}), 6.67(\mathrm{ps} \mathrm{t}, J=1.5 \mathrm{~Hz}, 1 \mathrm{H}), 6.55(\mathrm{~d}, J=8.0 \mathrm{~Hz}, 1 \mathrm{H}), 6.25(\mathrm{~d}, J=7.5 \mathrm{~Hz}, 2 \mathrm{H}), 4.16(\mathrm{dd}, J=$ $10.0,7.5 \mathrm{~Hz}, 1 \mathrm{H}$ ), 3.73 (ps t, $J=10.0 \mathrm{~Hz}, 1 \mathrm{H}), 3.59(\mathrm{dd}, J=10.0,7.5 \mathrm{~Hz}, 1 \mathrm{H}), 2.53(\mathrm{~s}, 3 \mathrm{H}), 2.05-1.92(\mathrm{~m}, 2 \mathrm{H}), 0.97(\mathrm{t}, J=$ $7.5 \mathrm{~Hz}, 3 \mathrm{H})$.

${ }^{13} \mathrm{C}$ NMR (125 MHz, $\mathrm{CDCl}_{3}$ ): $\delta$ (ppm) 175.3, 145.7, 137.9, 136.6, 135.2, 132.1, 131.0, 130.0, 129.6, 128.6, 128.1, 127.7, $127.5,122.2,59.3,48.2,47.7,28.0,21.9,8.9$.

MS (ESI) $m / z 498.0[\mathrm{M}+\mathrm{H}]^{+}, 519.6[\mathrm{M}+\mathrm{Na}]^{+}$.

HRMS (ESI) $\mathrm{m} / \mathrm{z}[\mathrm{M}+\mathrm{H}]^{+}$calcd for $\mathrm{C}_{25} \mathrm{H}_{25} \mathrm{BrNO}_{3} \mathrm{~S}$ 498.0733; Found 498.0733.

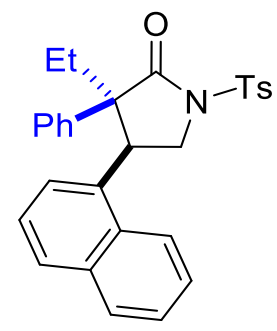

$3 \mathbf{x}$

\section{3-Ethyl-4-(naphthalen-1-yl)-3-phenyl-1-tosylpyrrolidin-2-one (3x)}

Procedure B: imine $1 \mathrm{j}$ (79.5 mg, $0.257 \mathrm{mmol})$ and ethylphenylketene $(75.0 \mathrm{mg}, 0.514 \mathrm{mmol})$ afforded $107.0 \mathrm{mg}(89 \%)$ of $y$-lactam $3 \mathbf{x}$ as a foam solid

m.p. $173-175^{\circ} \mathrm{C}$.

IR $v_{\max }$ (neat): $2988,2900,1735,1511,1369,1228,1171,1078 \mathrm{~cm}^{-1}$.

${ }^{1} \mathrm{H}$ NMR $\left(500 \mathrm{MHz}, \mathrm{CDCl}_{3}\right): \delta(\mathrm{ppm}) 8.13(\mathrm{~d}, J=9.0 \mathrm{~Hz}, 1 \mathrm{H}), 8.08(\mathrm{~d}, J=8.5 \mathrm{~Hz}, 2 \mathrm{H}), 7.83(\mathrm{~d}, J=7.0 \mathrm{~Hz}, 1 \mathrm{H}), 7.64(\mathrm{~d}, J=$ $8.0 \mathrm{~Hz}, 1 \mathrm{H}), 7.58-7.54(\mathrm{~m}, 1 \mathrm{H}), 7.51-7.48(\mathrm{~m}, 1 \mathrm{H}), 7.45(\mathrm{~d}, J=8.0 \mathrm{~Hz}, 2 \mathrm{H}), 7.01$ (ps t, $J=7.0 \mathrm{~Hz}, 1 \mathrm{H}), 6.97$ (ps t, $J=7.5 \mathrm{~Hz}$, $1 \mathrm{H}), 6.85(\mathrm{ps} \mathrm{t}, J=8.0 \mathrm{~Hz}, 2 \mathrm{H}), 6.29(\mathrm{~d}, J=7.5 \mathrm{~Hz}, 2 \mathrm{H}), 6.25(\mathrm{~d}, J=7.0 \mathrm{~Hz}, 1 \mathrm{H}), 4.62($ ps t, $J=7.5 \mathrm{~Hz}, 1 \mathrm{H}, \mathrm{CH}), 4.26$ (dd, J $=10.0,7.5 \mathrm{~Hz}, 1 \mathrm{H}$ ), $3.92(\mathrm{pst}, J=10.0 \mathrm{~Hz}, 1 \mathrm{H}), 2.54(\mathrm{~s}, 3 \mathrm{H}), 2.13-2.120(\mathrm{~m}, 2 \mathrm{H}), 0.97(\mathrm{t}, J=7.5 \mathrm{~Hz}, 3 \mathrm{H})$. 
$\left.{ }^{13} \mathrm{C} \mathrm{NMR} \mathrm{(125} \mathrm{MHz,} \mathrm{CDCl}\right)$ ): $\delta$ (ppm) 175.8, 145.6, 137.3, 135.3, 133.9, 132.6, 132.0, 130.0, 129.4, 128.6, 128.1, 127.9, $127.8,127.01,126.9,126.5,125.8,124.6,122.6,59.6,49.9,42.3,29.7,22.0,9.1$.

MS (ESI) $m / z 469.8[\mathrm{M}+\mathrm{H}]^{+}, 491.8[\mathrm{M}+\mathrm{Na}]^{+}$.

HRMS (ESI) $\mathrm{m} / \mathrm{z}[\mathrm{M}+\mathrm{H}]^{+}$calcd for $\mathrm{C}_{29} \mathrm{H}_{28} \mathrm{NO}_{3} \mathrm{~S} 470.1784$; Found 470.1787.

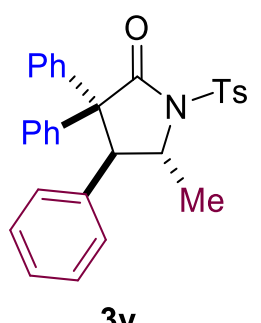

3y

(4,5-trans)-5-Methyl-3,3,4-triphenyl-1-tosylpyrrolidin-2-one (3y)

Procedure B: imine 1a (60 mg, $0.231 \mathrm{mmol}, 1 \mathrm{eq}), 1,1$-diiodoethane $(47 \mu \mathrm{L}, 0.463 \mathrm{mmol}, 2 \mathrm{eq})$ and diphenylketene (180 mg, $0.925 \mathrm{mmol}, 4 \mathrm{eq}$ ) afforded $85 \mathrm{mg}(76 \%)$ of $\gamma$-lactam $3 \mathbf{y}$ in a 76:24 mixture with cis-aziridine $\mathbf{2 p}$-cis, not separable. (A small fraction of pure $3 y$ was isolated for NMR)

IR $\boldsymbol{v}_{\max }$ (neat): $3059,3047,1732,1597,1494,1445,1359,1170,1088 \mathrm{~cm}^{-1}$.

${ }^{1} \mathrm{H}$ NMR $\left(400 \mathrm{MHz}, \mathrm{CDCl}_{3}\right): \delta(\mathrm{ppm}) 8.05(\mathrm{~d}, J=8.4 \mathrm{~Hz}, 2 \mathrm{H}), 7.46(\mathrm{~d}, J=7.2 \mathrm{~Hz}, 2 \mathrm{H}), 7.37(\mathrm{~d}, J=8.4 \mathrm{~Hz}, 2 \mathrm{H}), 7.31-7.24$ $(\mathrm{m}, 4 \mathrm{H}), 7.18-7.12(\mathrm{~m}, 1 \mathrm{H}), 7.07$ (ps t, $J=7.2 \mathrm{~Hz}, 2 \mathrm{H}), 7.00$ (ps t, $J=7.2 \mathrm{~Hz}, 2 \mathrm{H}), 6.67(\mathrm{~d}, J=7.2 \mathrm{~Hz}, 2 \mathrm{H}), 6.55(\mathrm{~d}, J=7.2$ $\mathrm{Hz}, 2 \mathrm{H}), 4.42-4.36(\mathrm{~m}, 1 \mathrm{H}), 4.07(\mathrm{~d}, J=8.8 \mathrm{~Hz}, 1 \mathrm{H}), 2.47(\mathrm{~s}, 3 \mathrm{H}) 1.53(\mathrm{~d}, J=5.6 \mathrm{~Hz}, 3 \mathrm{H})$.

${ }^{13} \mathrm{C}$ NMR (100 MHz, CDCl $)$ : $\delta$ (ppm) 175.2, 145.4, 140.9, 139.7, 136.3, 135.8, 130.3, 129.9, 129.7, 129.2, 129.0, 128.31, $128.32,127.92,127.86,127.7,127.4,63.0,58.8,56.1,22.0,20.1$.

MS (ESI) $m / z 482.0[\mathrm{M}+\mathrm{H}]^{+}$.

HRMS (ESI) $\mathrm{m} / \mathrm{z}[\mathrm{M}+\mathrm{H}]^{+}$calcd for $\mathrm{C}_{30} \mathrm{H}_{28} \mathrm{NO}_{2} \mathrm{~S} 482.1784$; Found 482.1787.

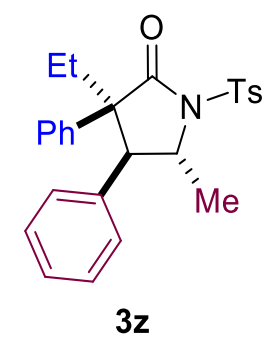

(4,5-trans)-3-Ethyl-5-methyl-3,4-diphenyl-1-tosylpyrrolidin-2-one (3z)

Procedure B: imine 1a (58 mg, $0.224 \mathrm{mmol}, 1 \mathrm{eq}), 1,1$-diiodoethane ( $45 \mu \mathrm{L}, 0.447 \mathrm{mmol}, 2 \mathrm{eq}$ ) and ethylphenylketene (131 mg, $0.896 \mathrm{mmol}, 4 \mathrm{eq}$ ) afforded $55 \mathrm{mg}(57 \%)$ of $\gamma$-lactam $3 z$ in a mixture with aziridine $\mathbf{2 p}$ in proportion 65:35 (3z: 2p-cis) not separable, as a colourless oil.

IR $\boldsymbol{V}_{\max }$ (neat): $3042,2998,1732,1596,1452,1448,1359,1173,1087 \mathrm{~cm}^{-1}$.

${ }^{1} \mathrm{H}$ NMR $\left(400 \mathrm{MHz}, \mathrm{CDCl}_{3}\right): \delta(\mathrm{ppm}) 8.11(\mathrm{~d}, J=8.4 \mathrm{~Hz}, 2 \mathrm{H}), 7.42(\mathrm{~d}, J=8.0 \mathrm{~Hz}, 2 \mathrm{H}), 7.21-7.05(\mathrm{~m}, 4 \mathrm{H}), 6.96$ (ps t, $J=7.6$ $\mathrm{Hz}, 2 \mathrm{H}), 6.58(\mathrm{~d}, J=7.2 \mathrm{~Hz}, 2 \mathrm{H}), 6.38(\mathrm{~d}, J=7.2 \mathrm{~Hz}, 2 \mathrm{H}), 4.37-4.25(\mathrm{~m}, 1 \mathrm{H}), 3.13(\mathrm{~d}, J=10.0 \mathrm{~Hz}, 1 \mathrm{H}), 2.50(\mathrm{~s}, 3 \mathrm{H}), 2.03-$ $1.86(\mathrm{~m}, 2 \mathrm{H}), 1.52(\mathrm{~d}, J=5.8 \mathrm{~Hz}, 3 \mathrm{H}), 0.98(\mathrm{t}, J=7.2 \mathrm{~Hz}, 3 \mathrm{H})$.

MS (APCI) $m / z 434.1[\mathrm{M}+\mathrm{H}]^{+}$.

HRMS (ESI) $\mathrm{m} / z[\mathrm{M}+\mathrm{H}]^{+}$calcd for $\mathrm{C}_{26} \mathrm{H}_{28} \mathrm{NO}_{3} \mathrm{~S}$ 434.1784; Found 434.1784. 


\section{NMR spectra}

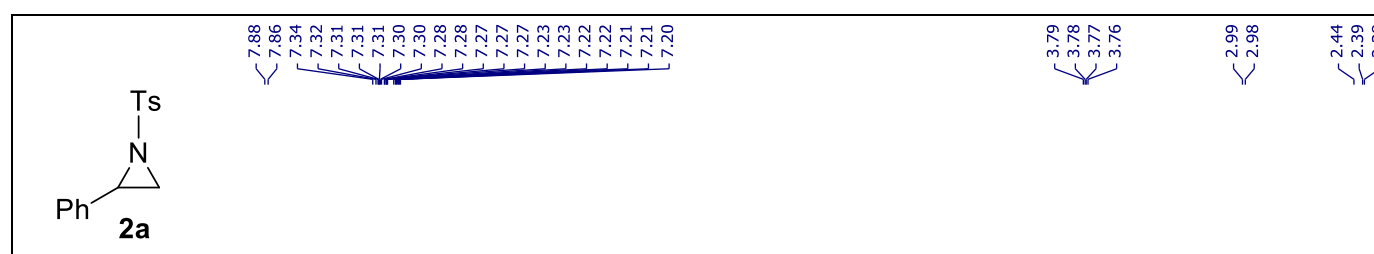

$\mathrm{RMN}{ }^{1} \mathrm{H}, \mathrm{CDCl}_{3}, 500 \mathrm{MHz}$

RMN ${ }^{13} \mathrm{C}, \mathrm{CDCl}_{3}, 125 \mathrm{MHz}$
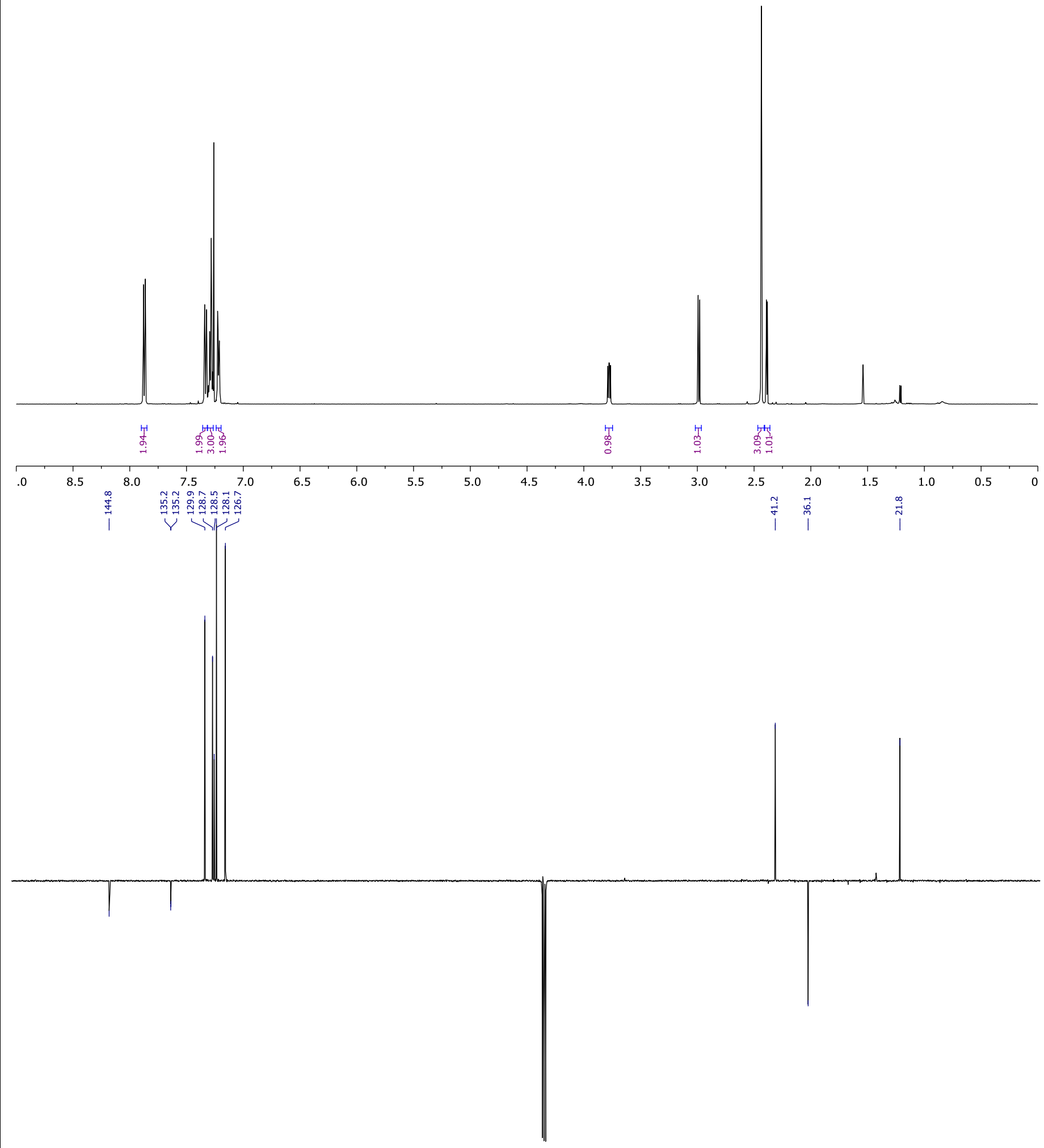


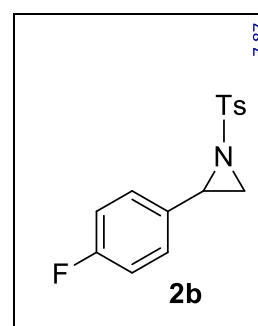

$\mathrm{RMN}{ }^{1} \mathrm{H}, \mathrm{CDCl}_{3}, 500 \mathrm{MHz}$

$\mathrm{RMN}{ }^{13} \mathrm{C}, \mathrm{CDCl}_{3}, 125 \mathrm{MHz}$

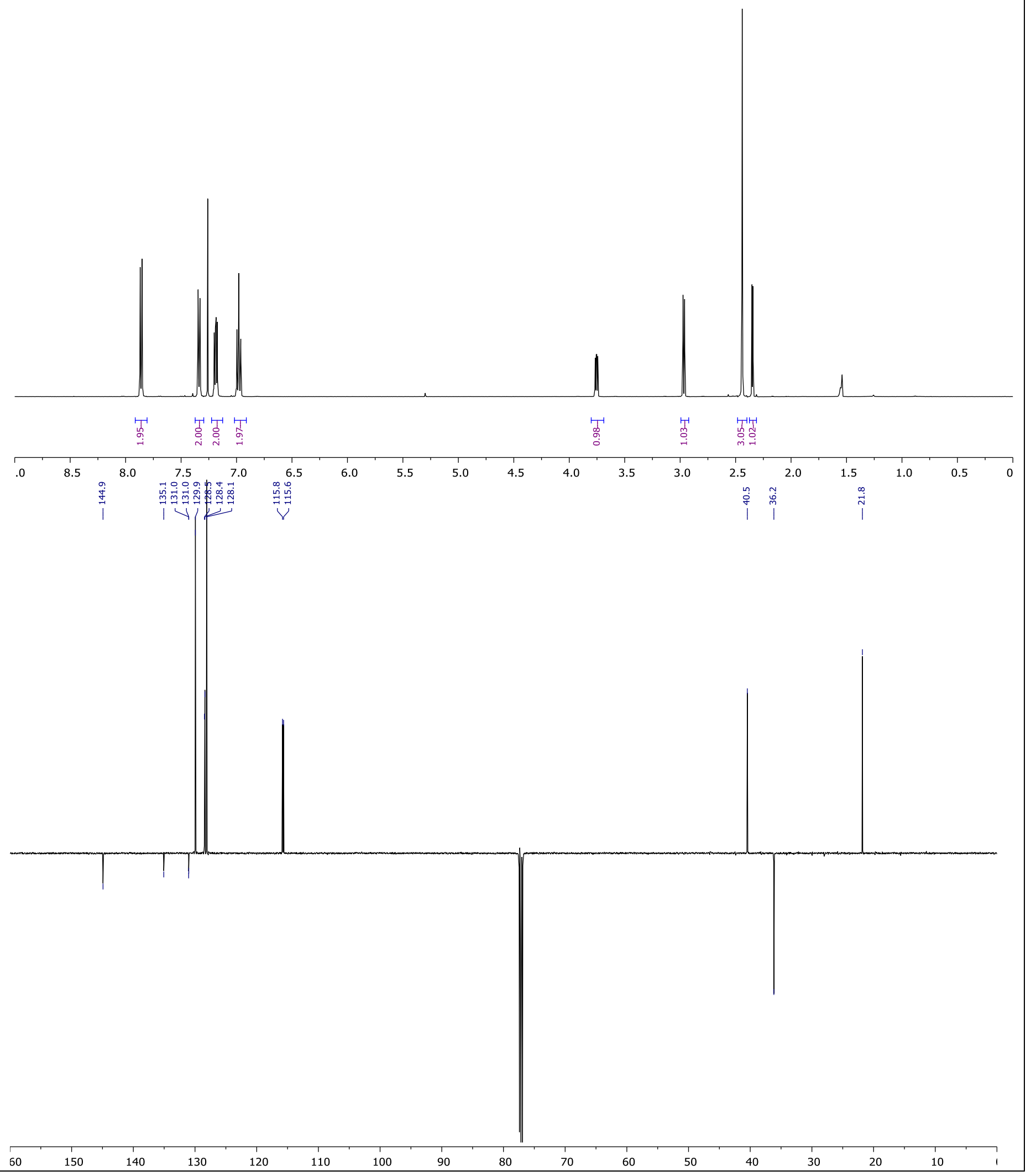




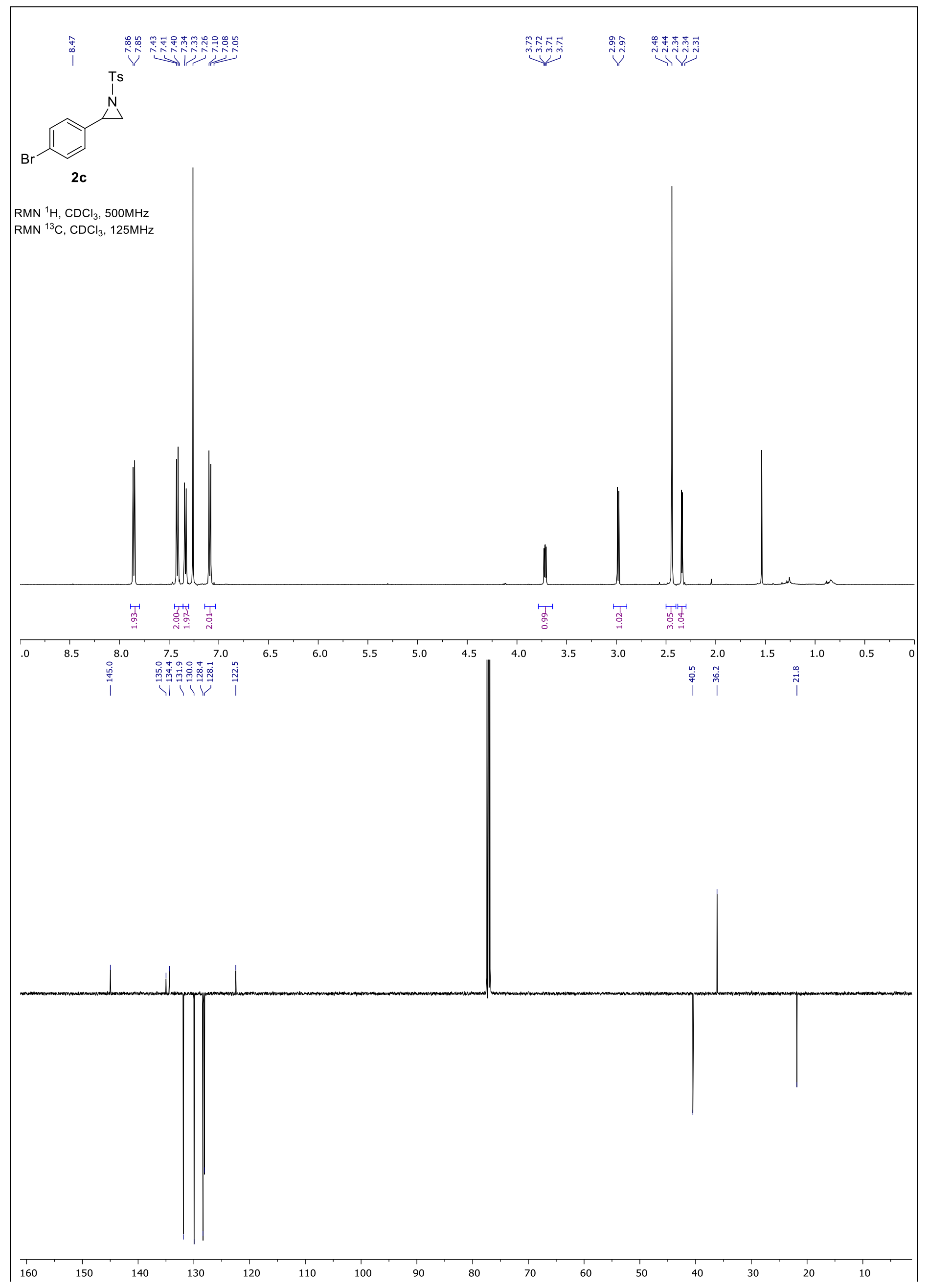




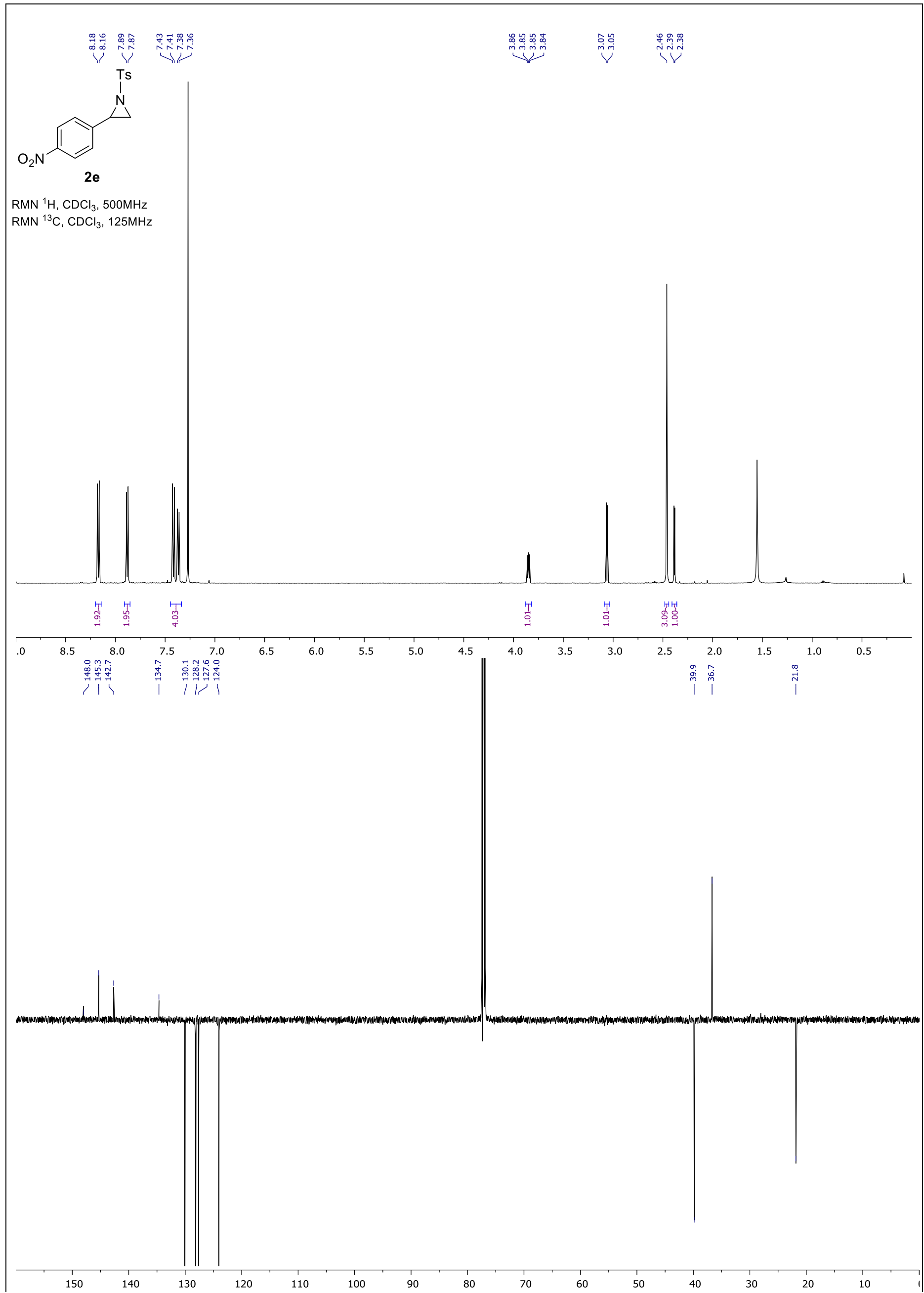




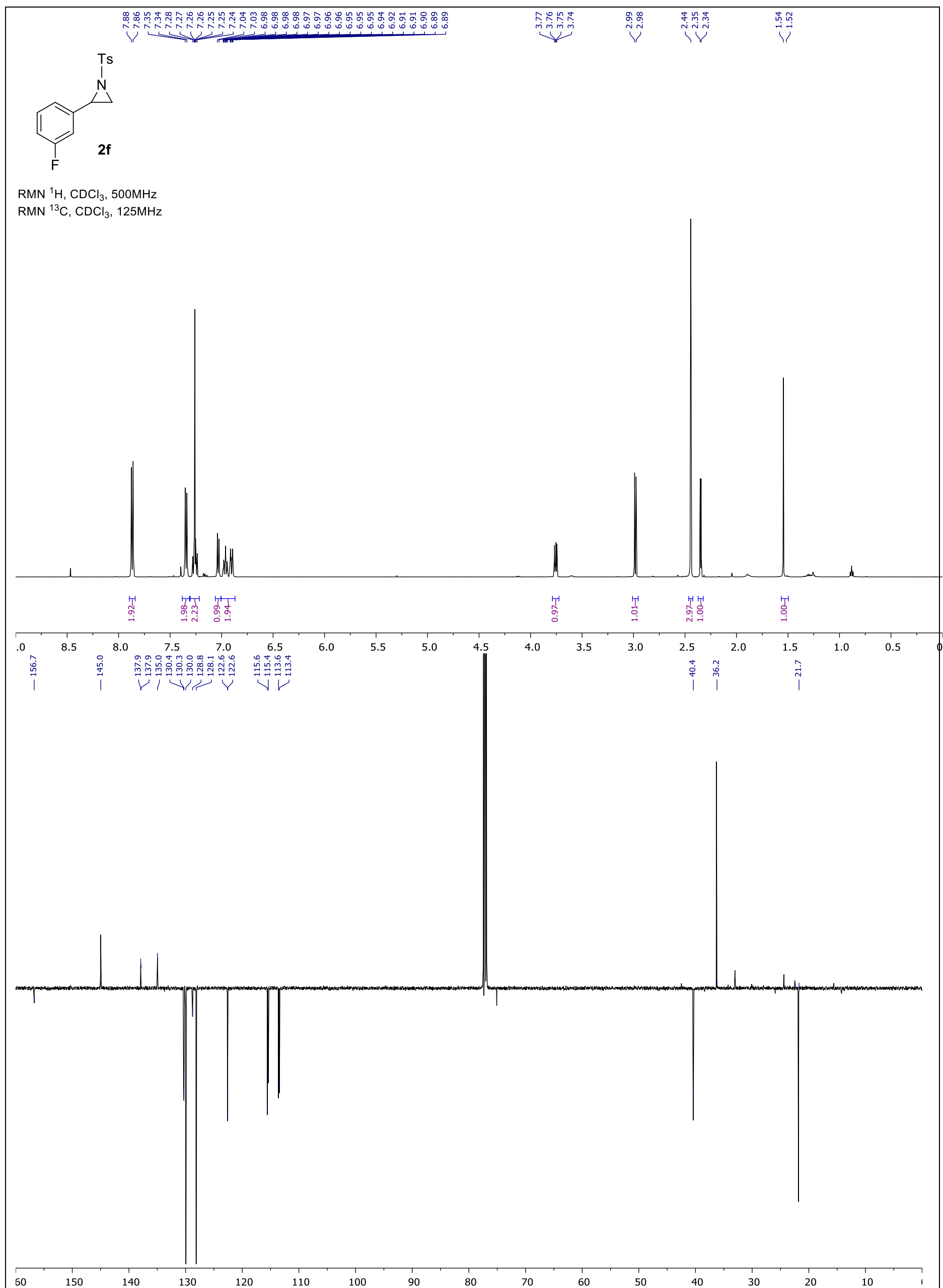




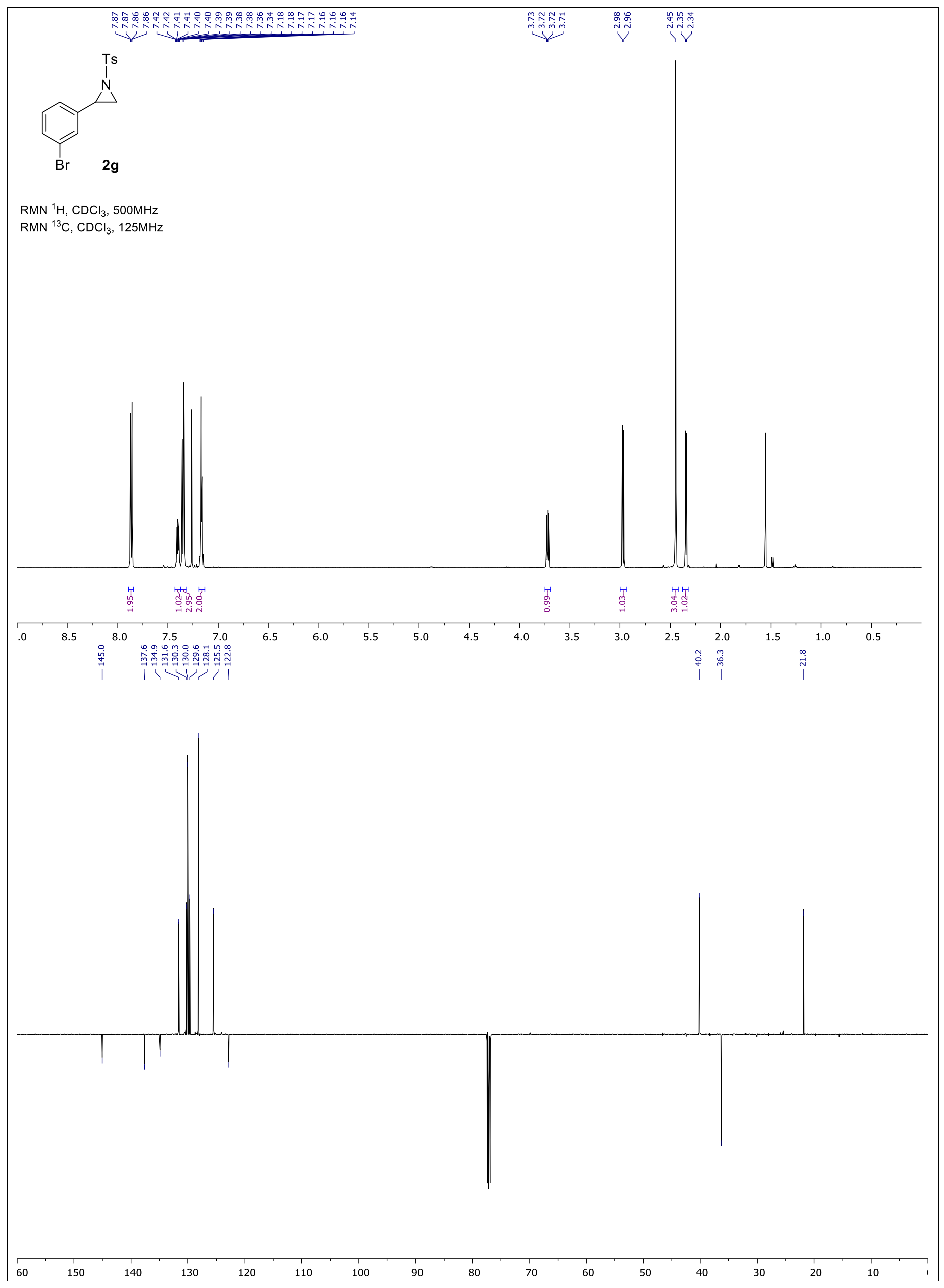




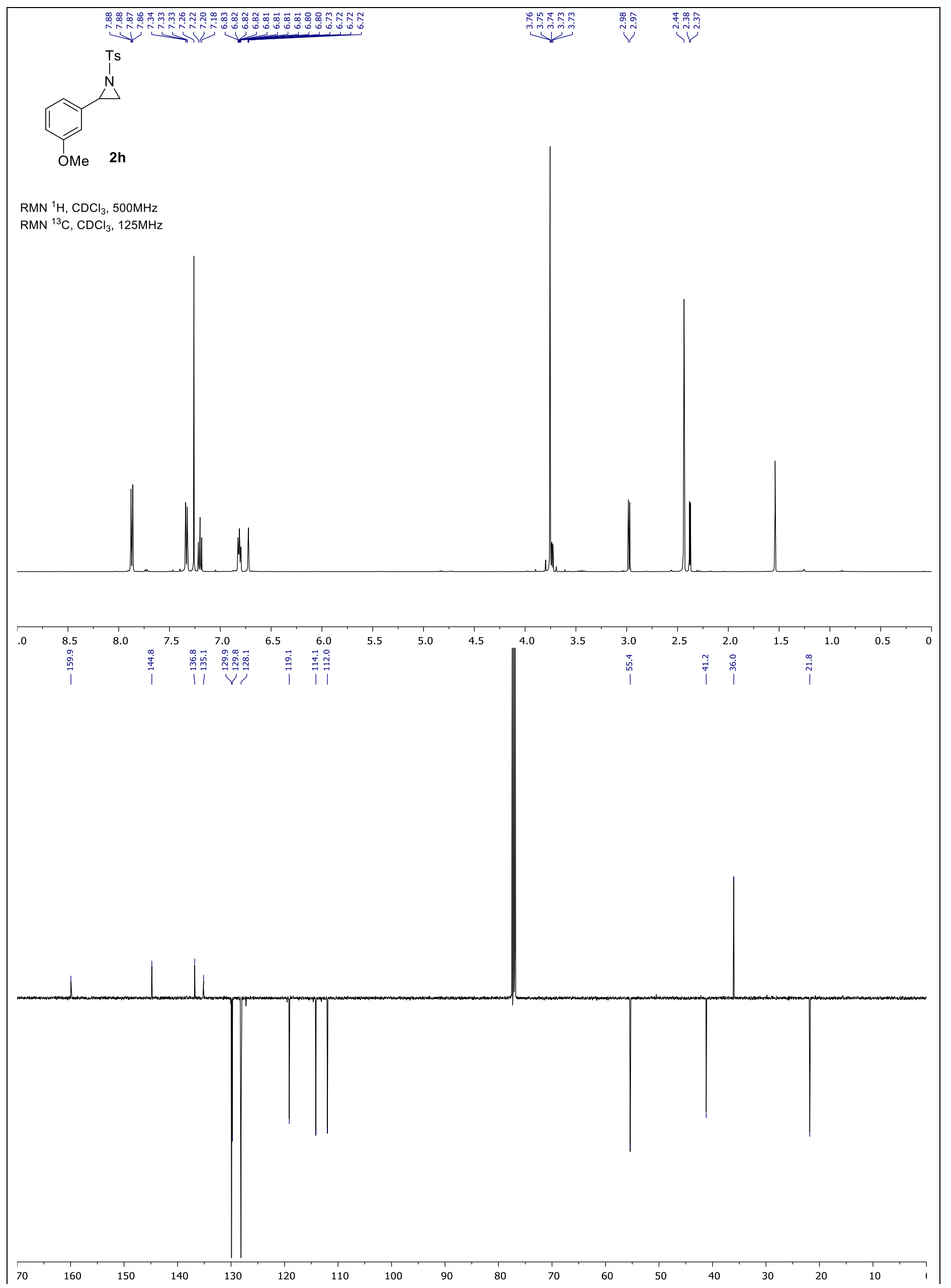




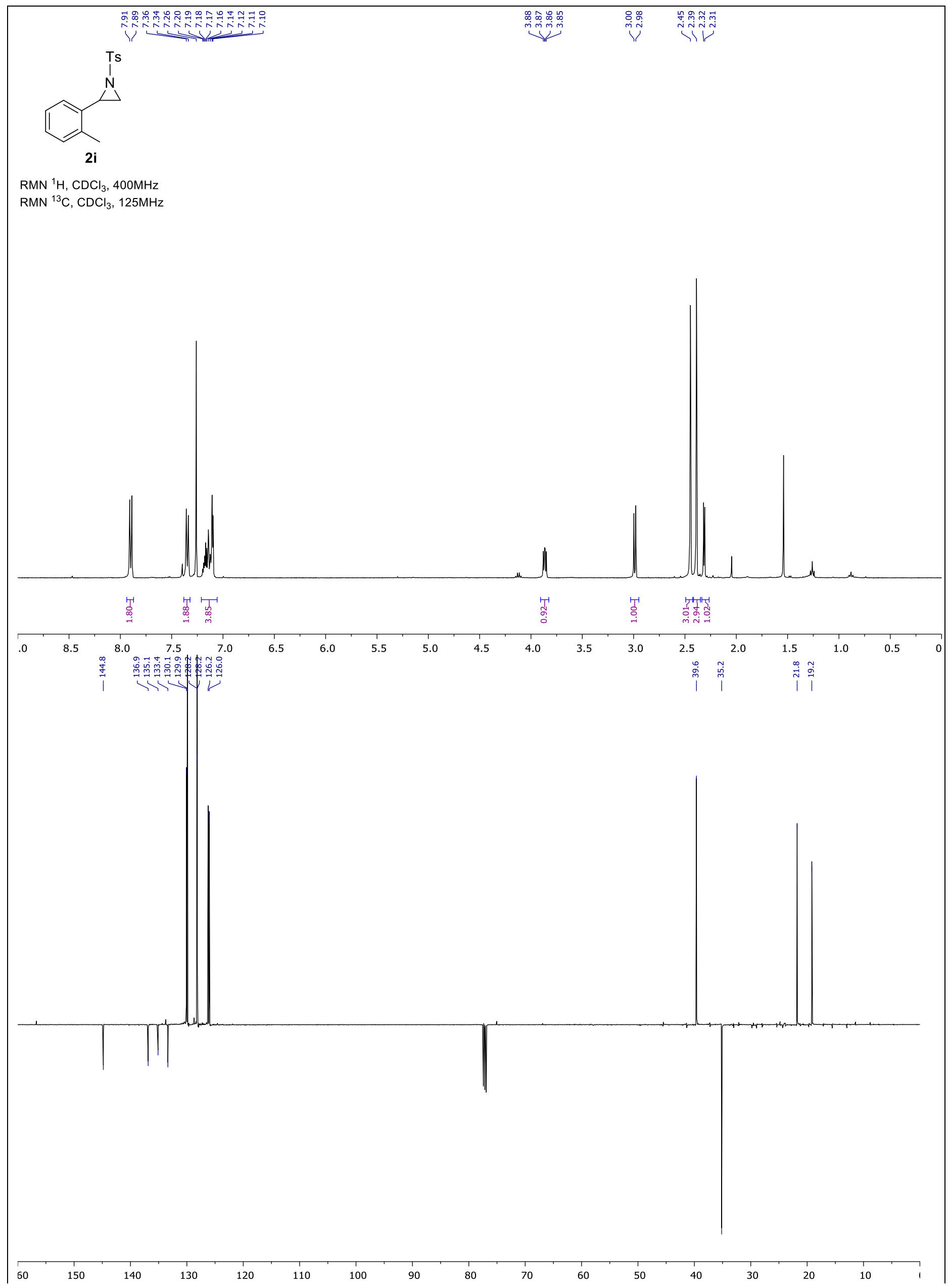




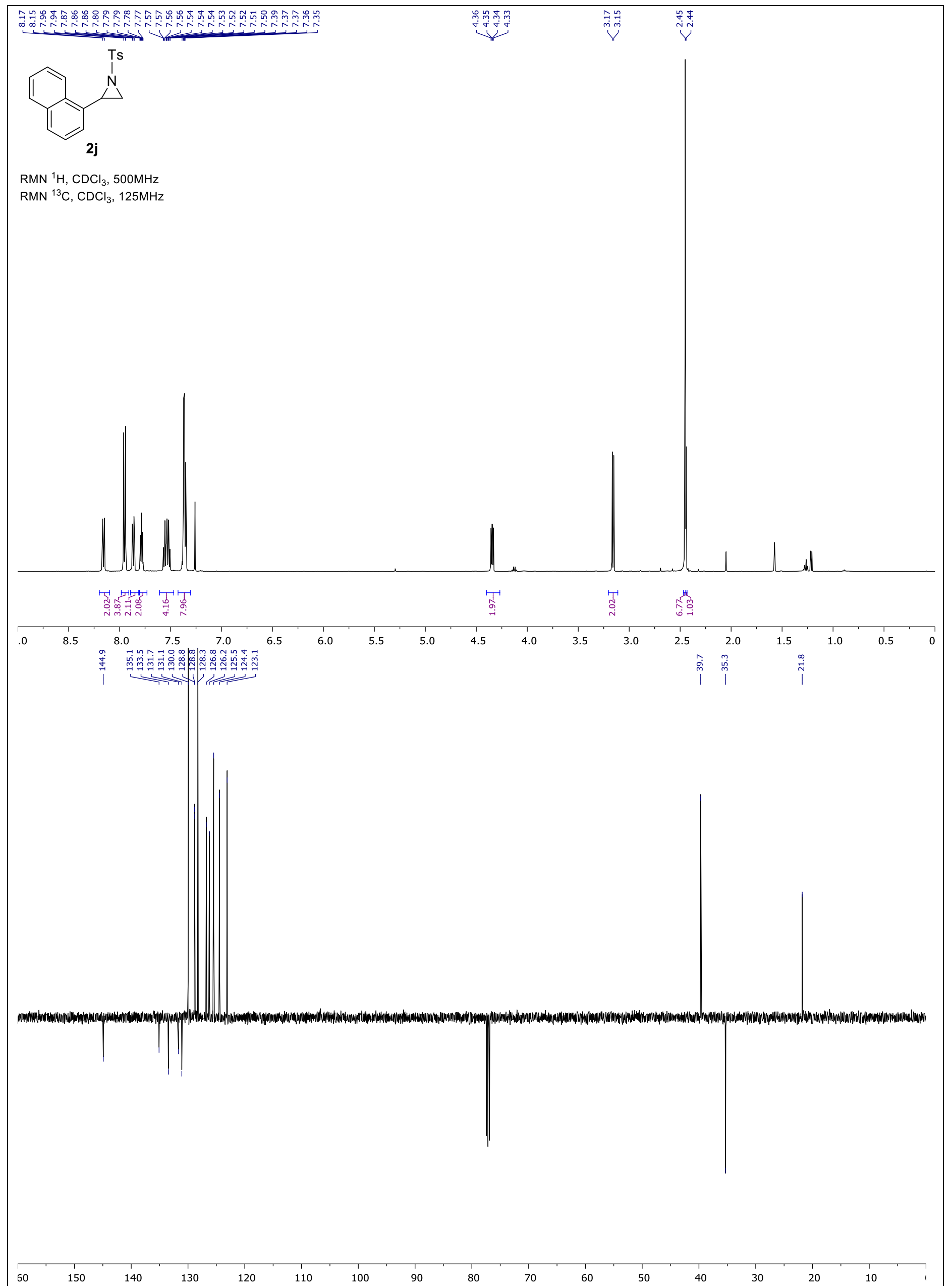




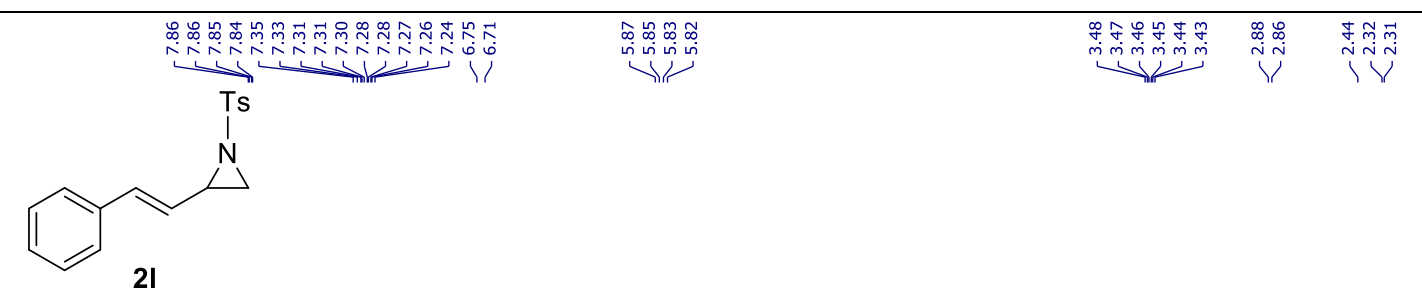

$\mathrm{RMN}{ }^{1} \mathrm{H}, \mathrm{CDCl}_{3}, 400 \mathrm{MHz}$

$\mathrm{RMN}{ }^{13} \mathrm{C}, \mathrm{CDCl}_{3}, 125 \mathrm{MHz}$
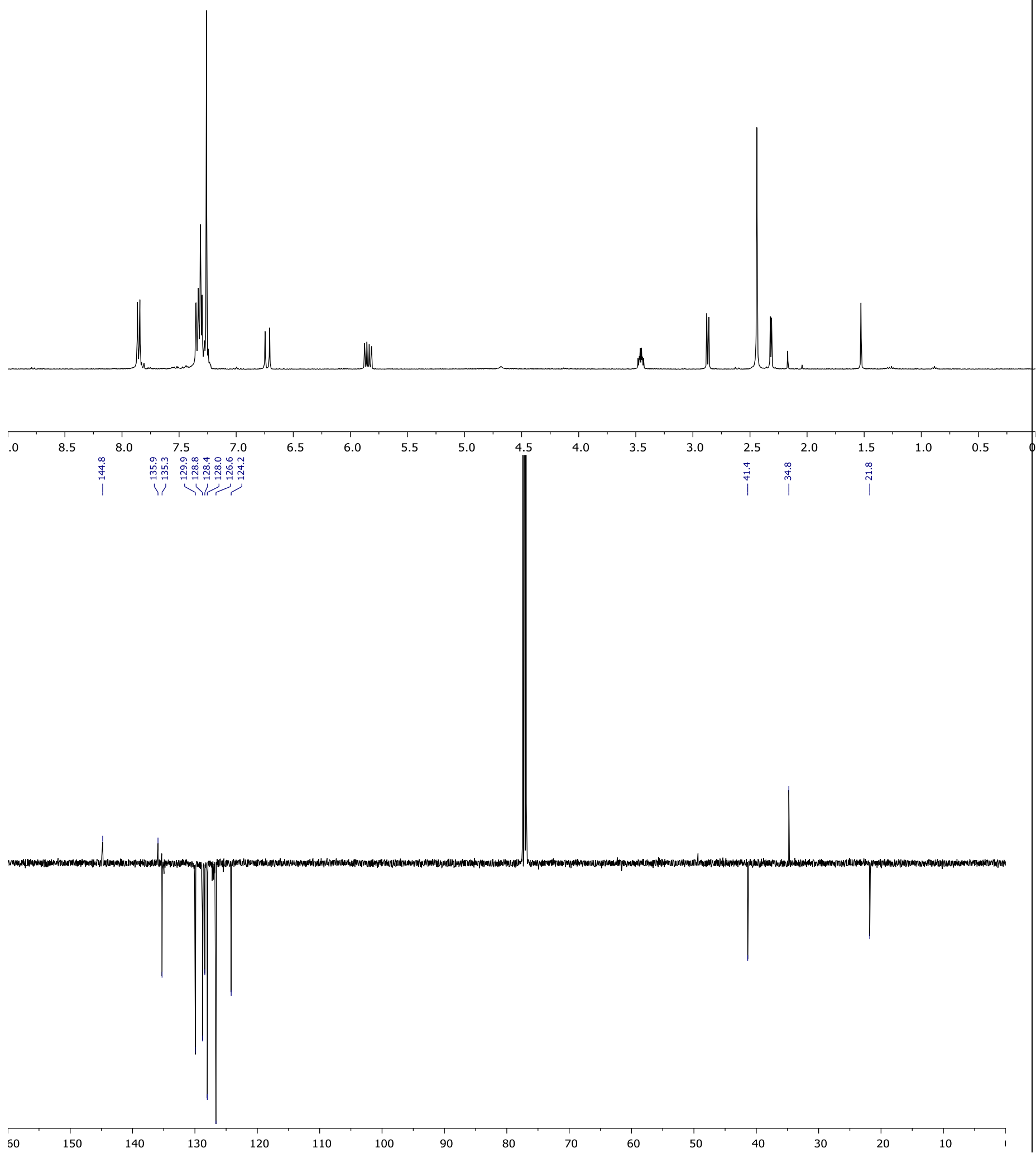


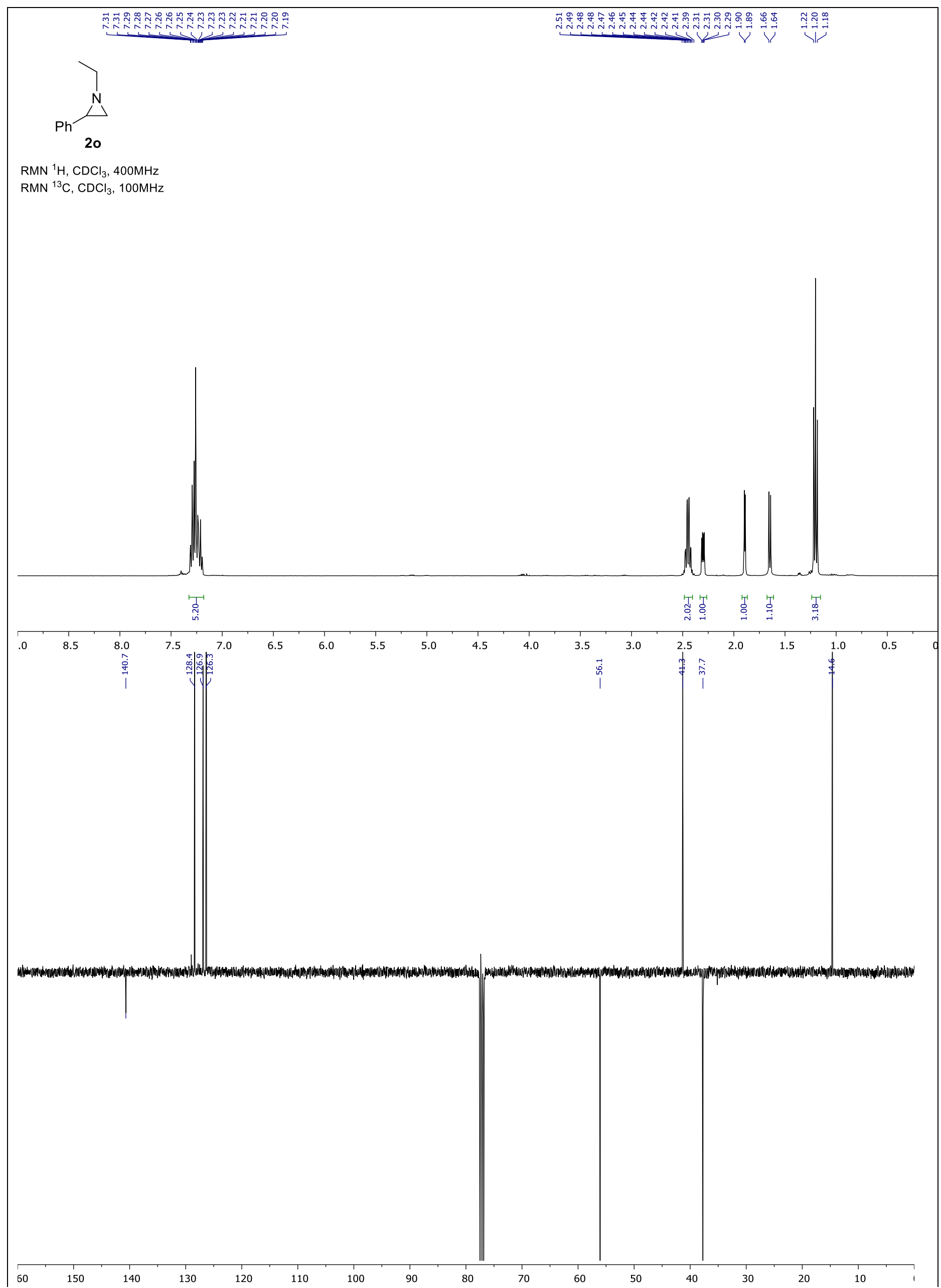




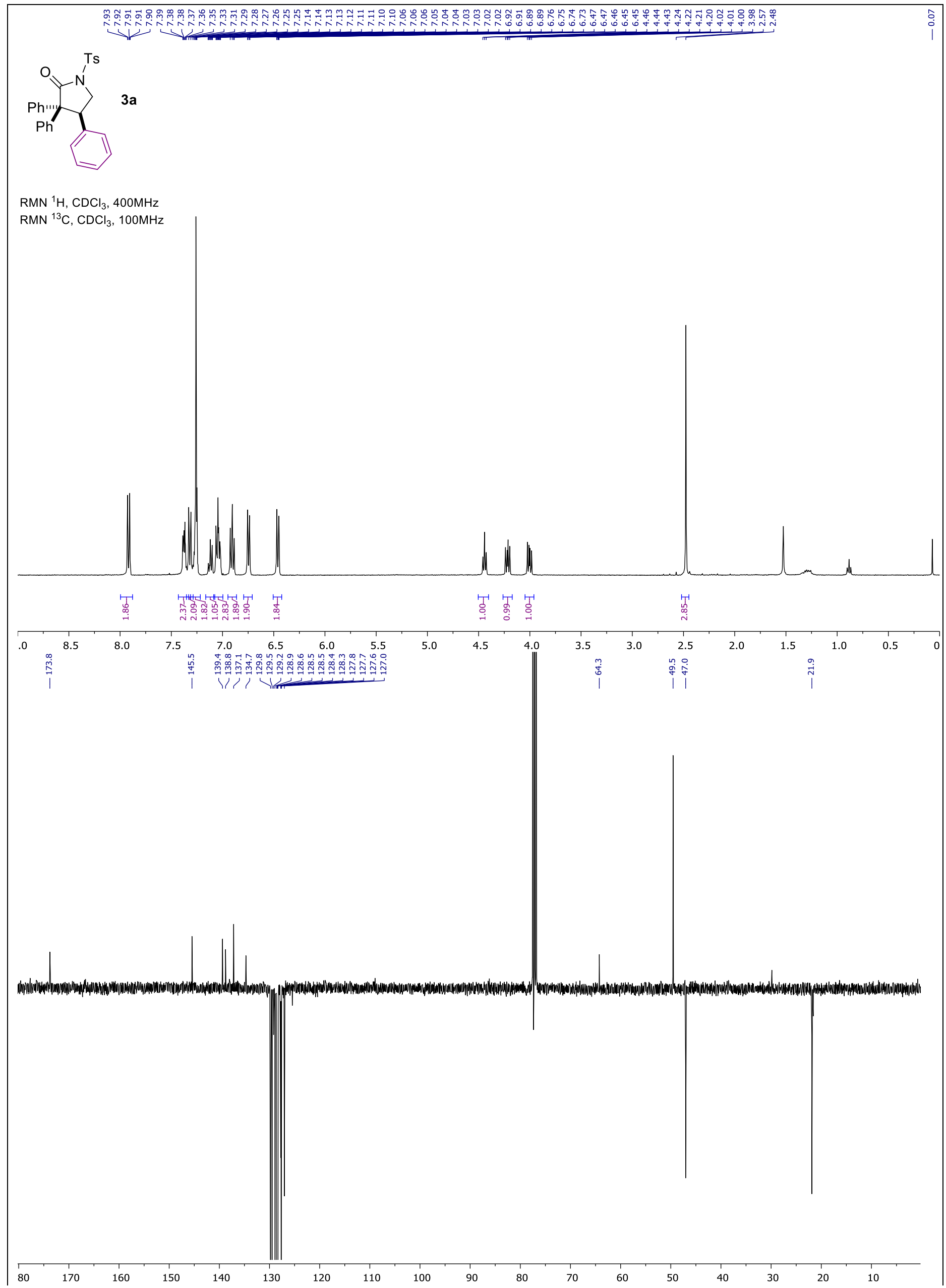




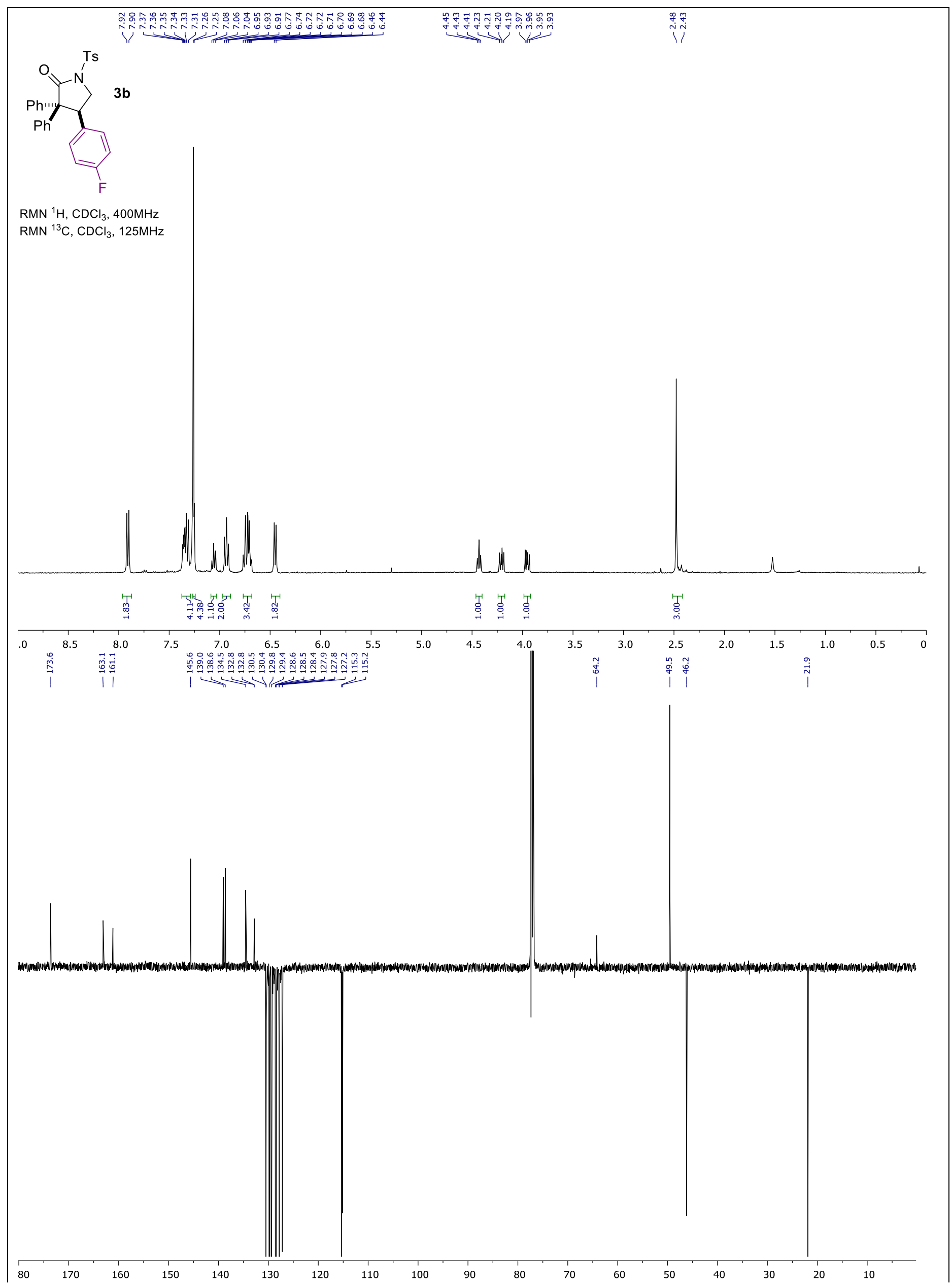




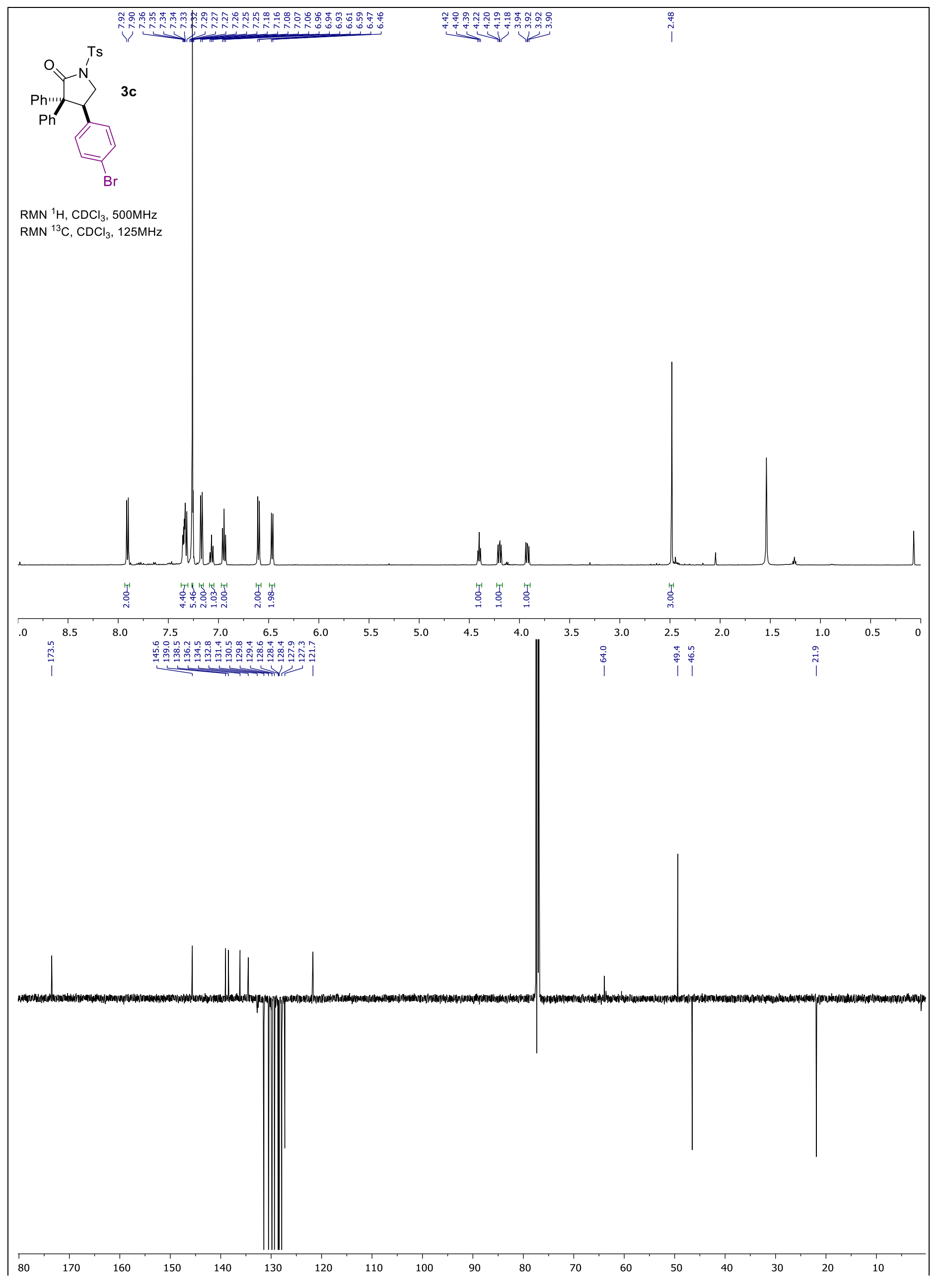

35 


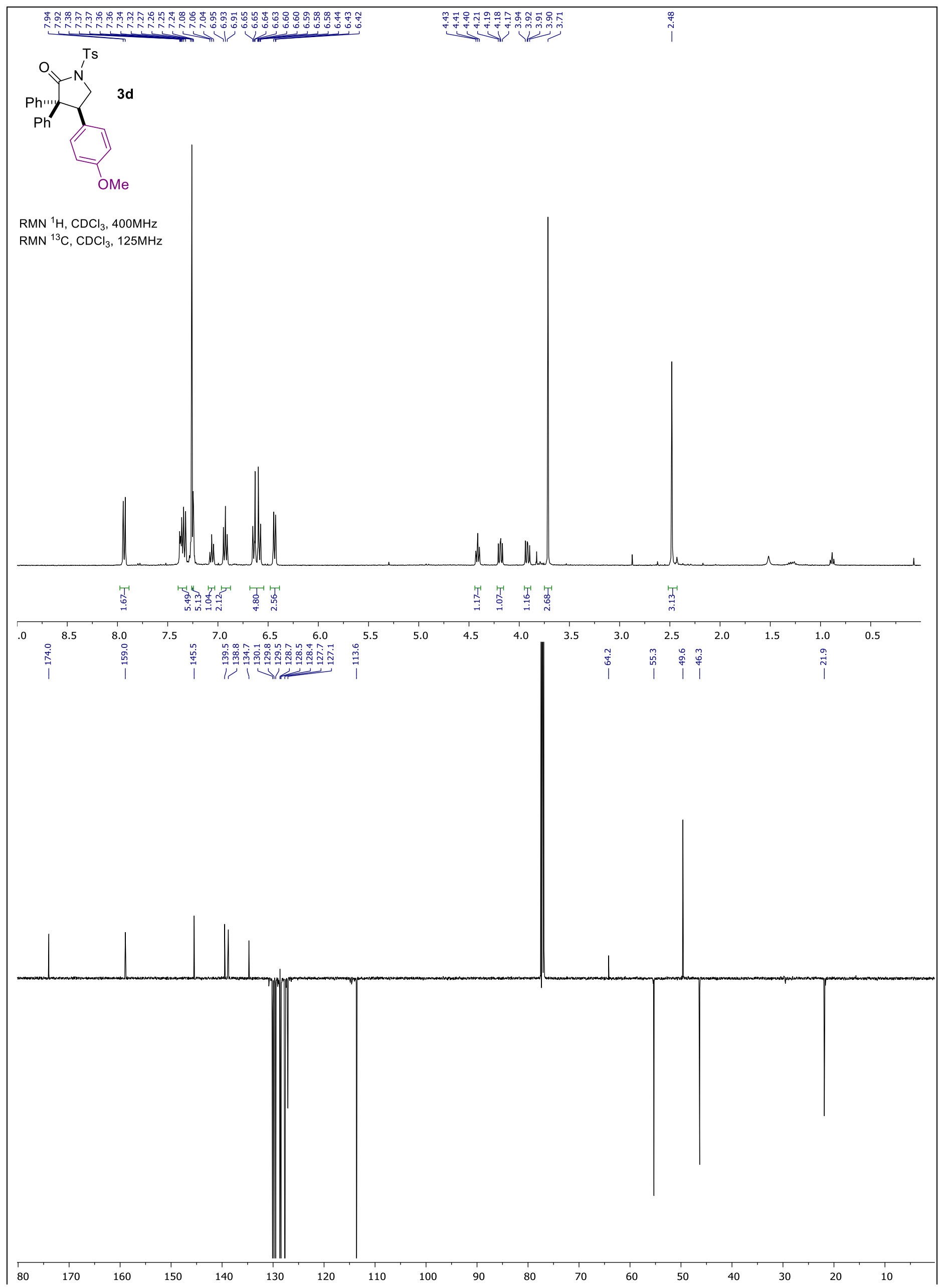




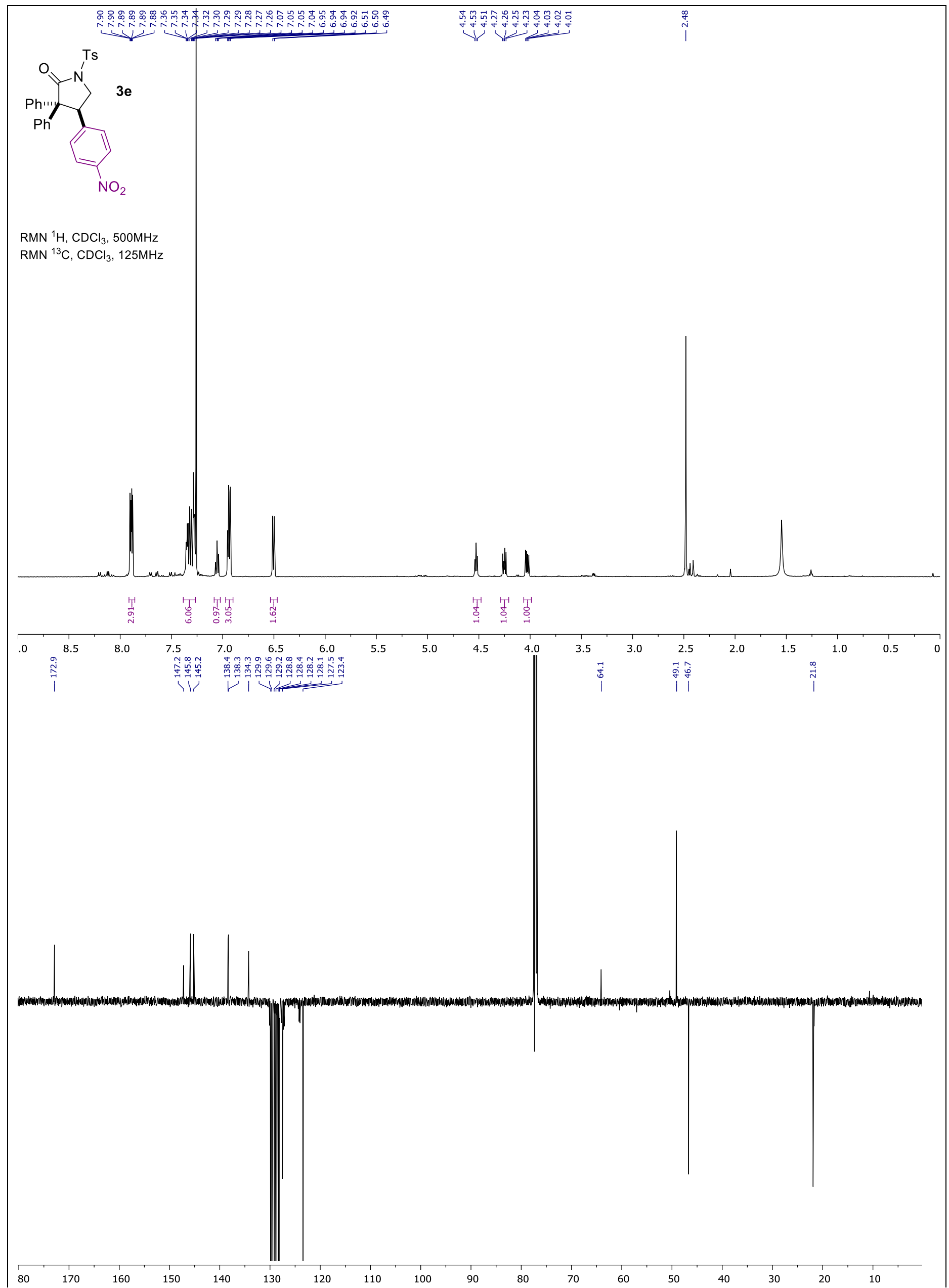




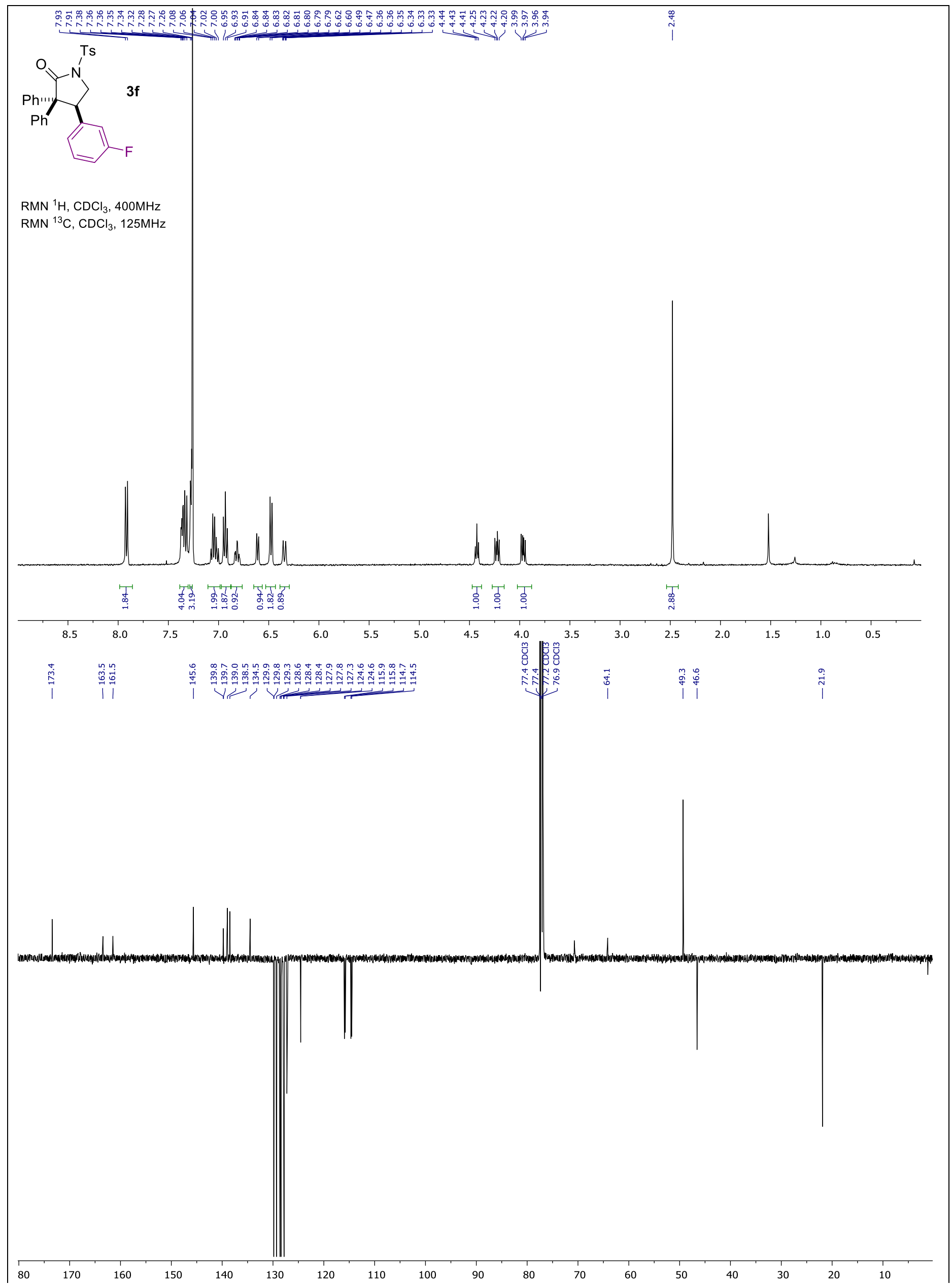




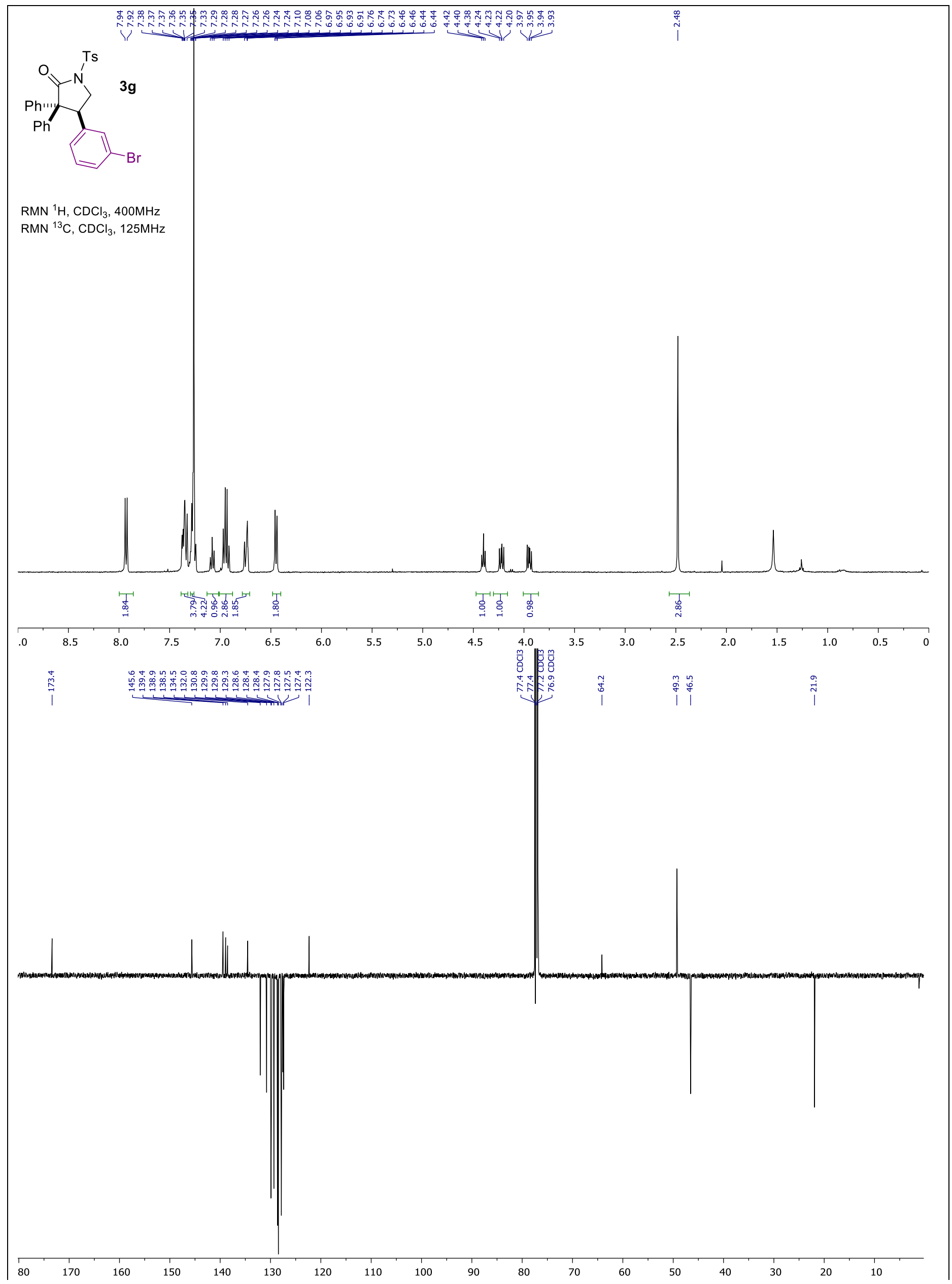




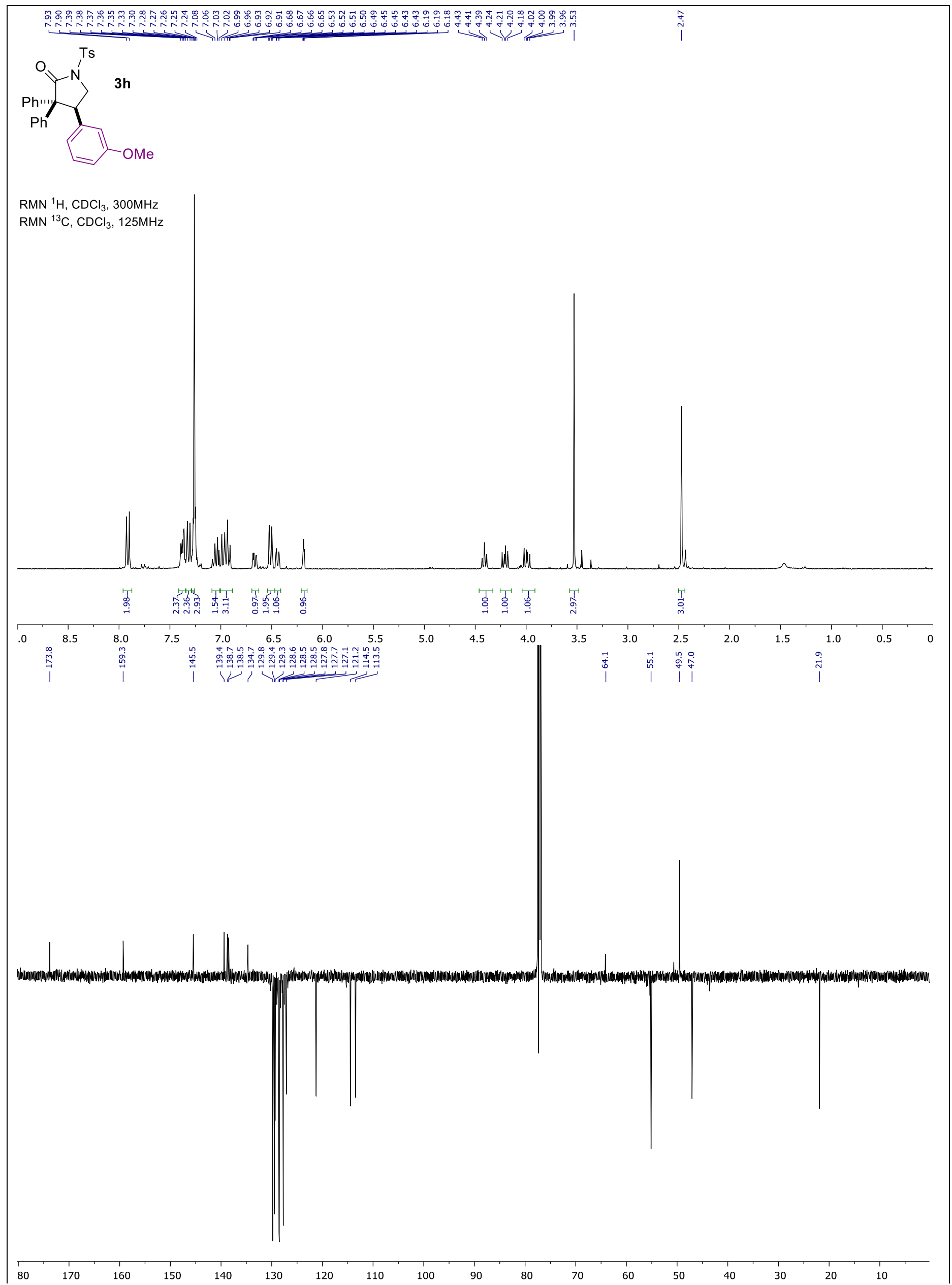




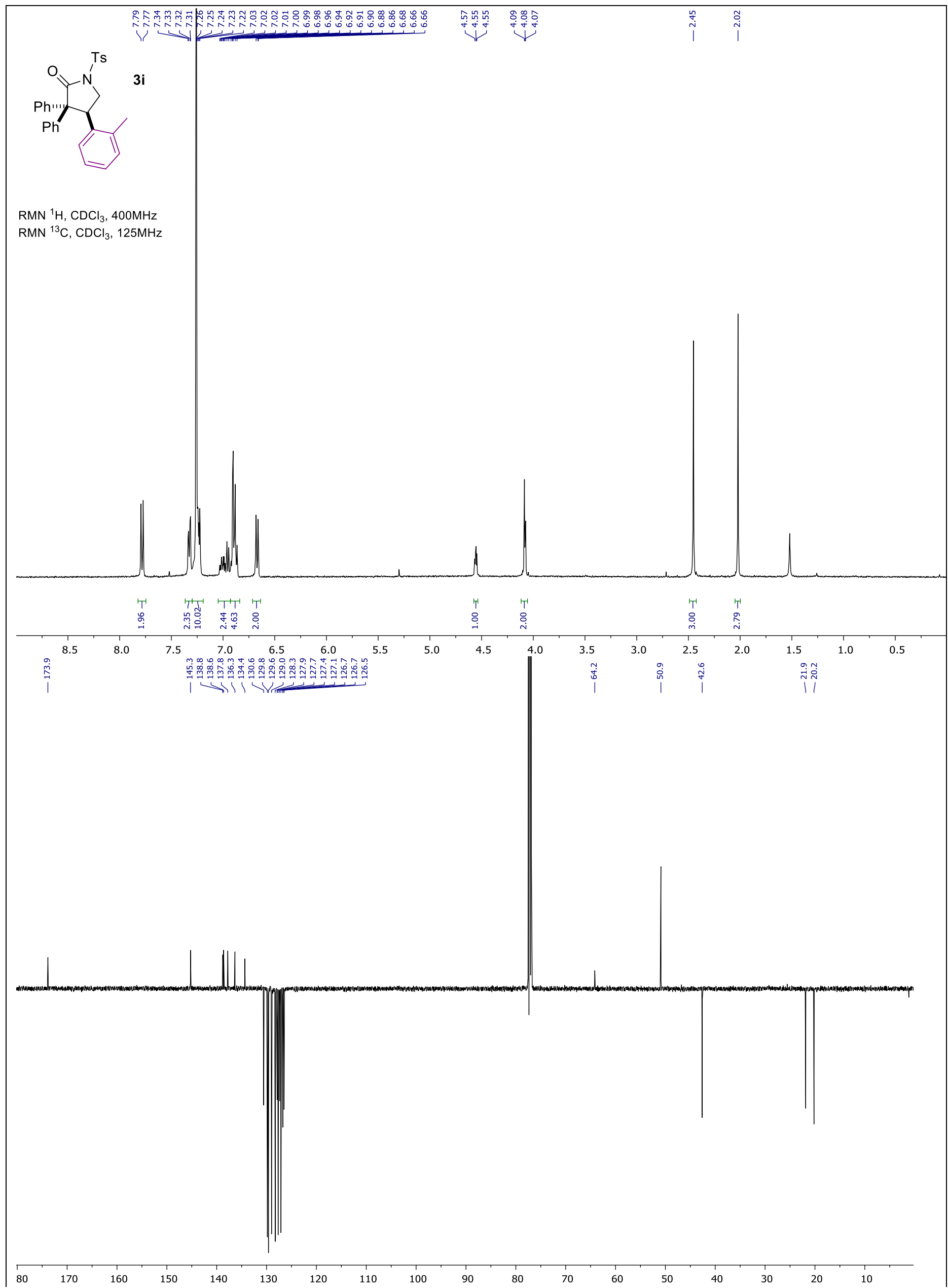




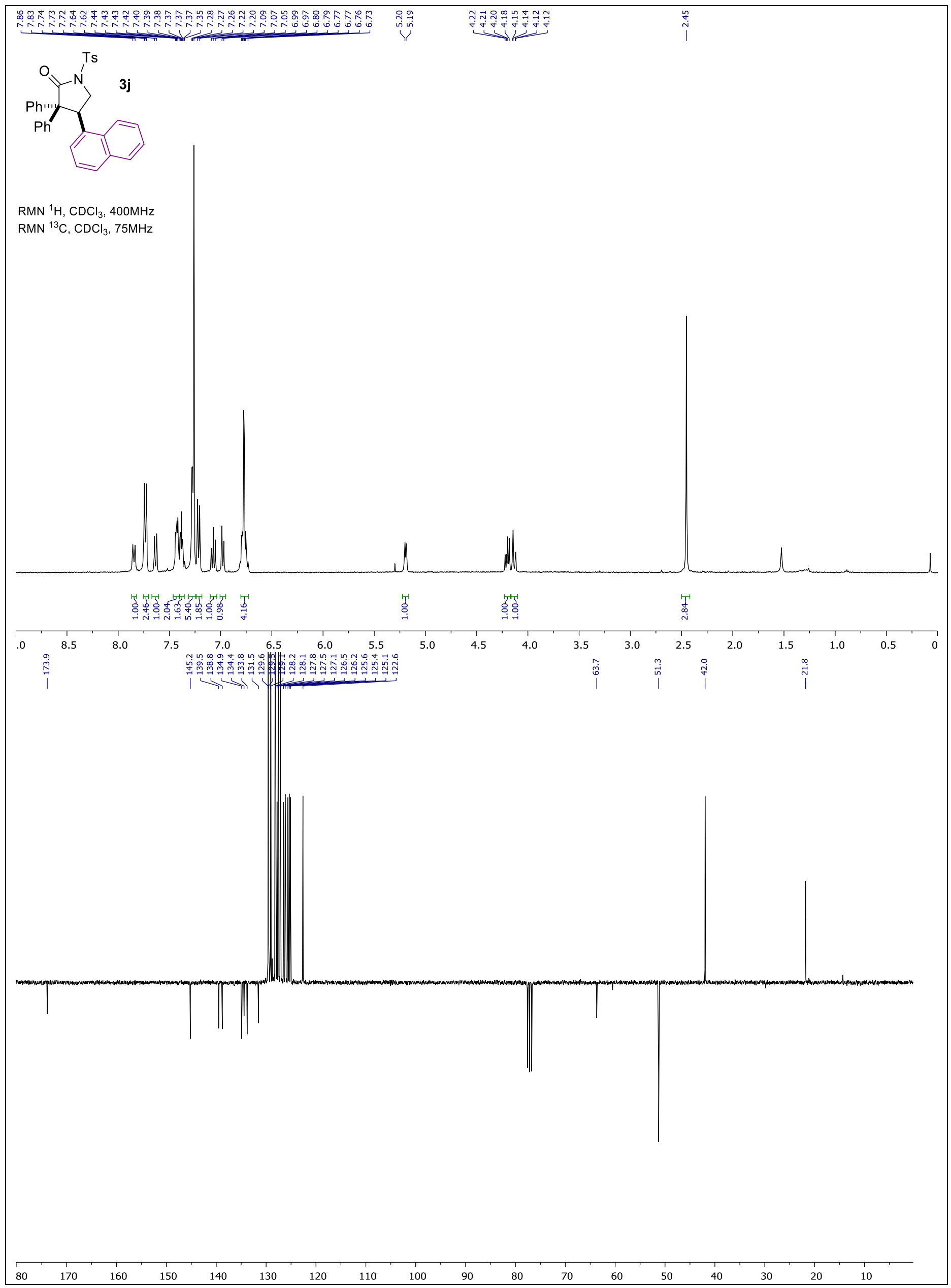




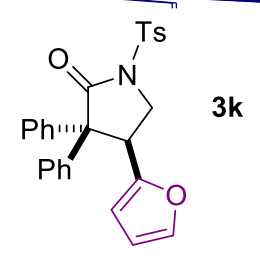

$\mathrm{RMN}{ }^{1} \mathrm{H}, \mathrm{CDCl}_{3}, 400 \mathrm{MHz}$

$\mathrm{RMN}{ }^{13} \mathrm{C}, \mathrm{CDCl}_{3}, 125 \mathrm{MHz}$

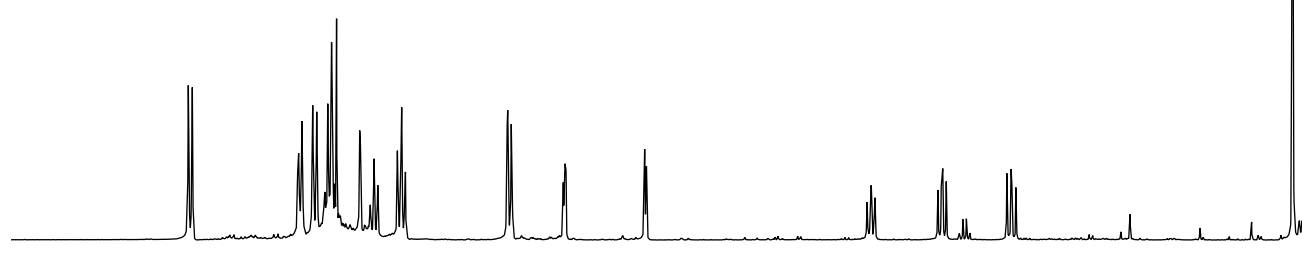

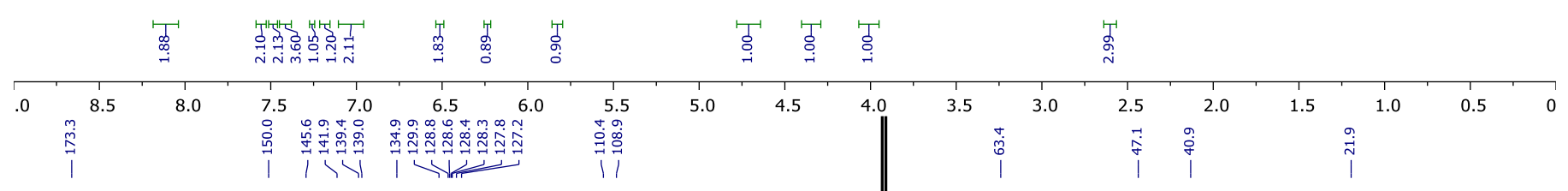

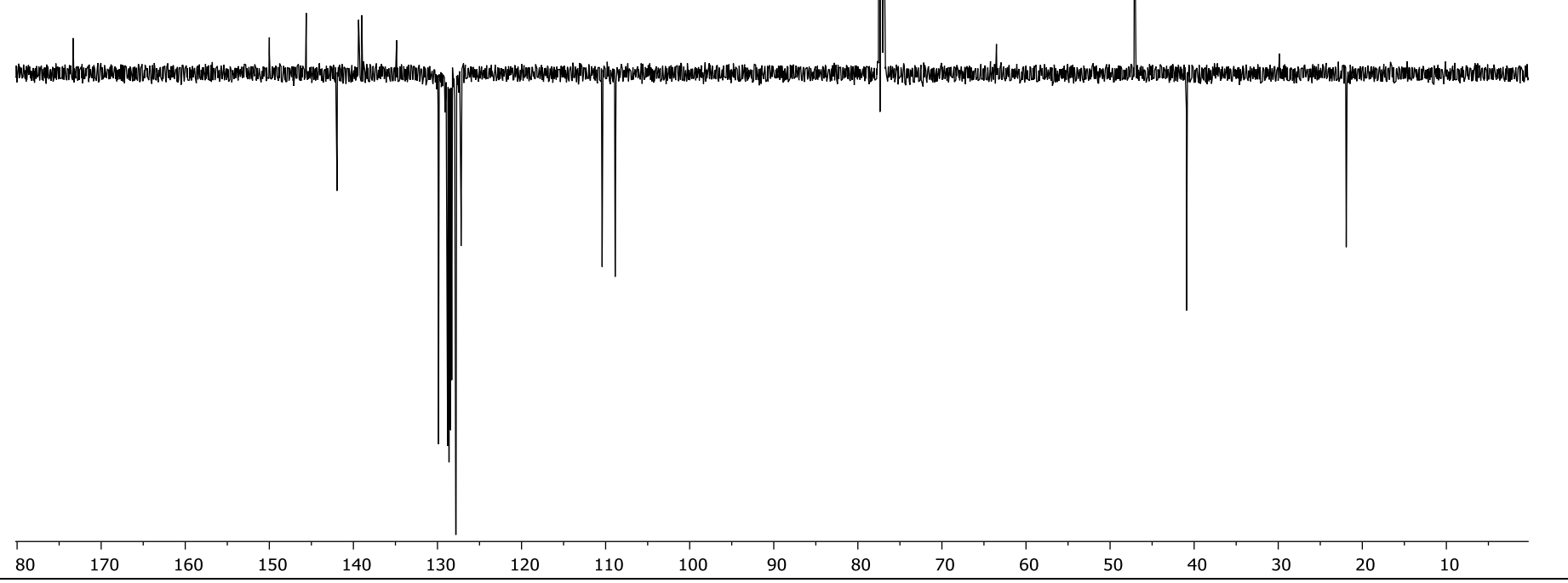




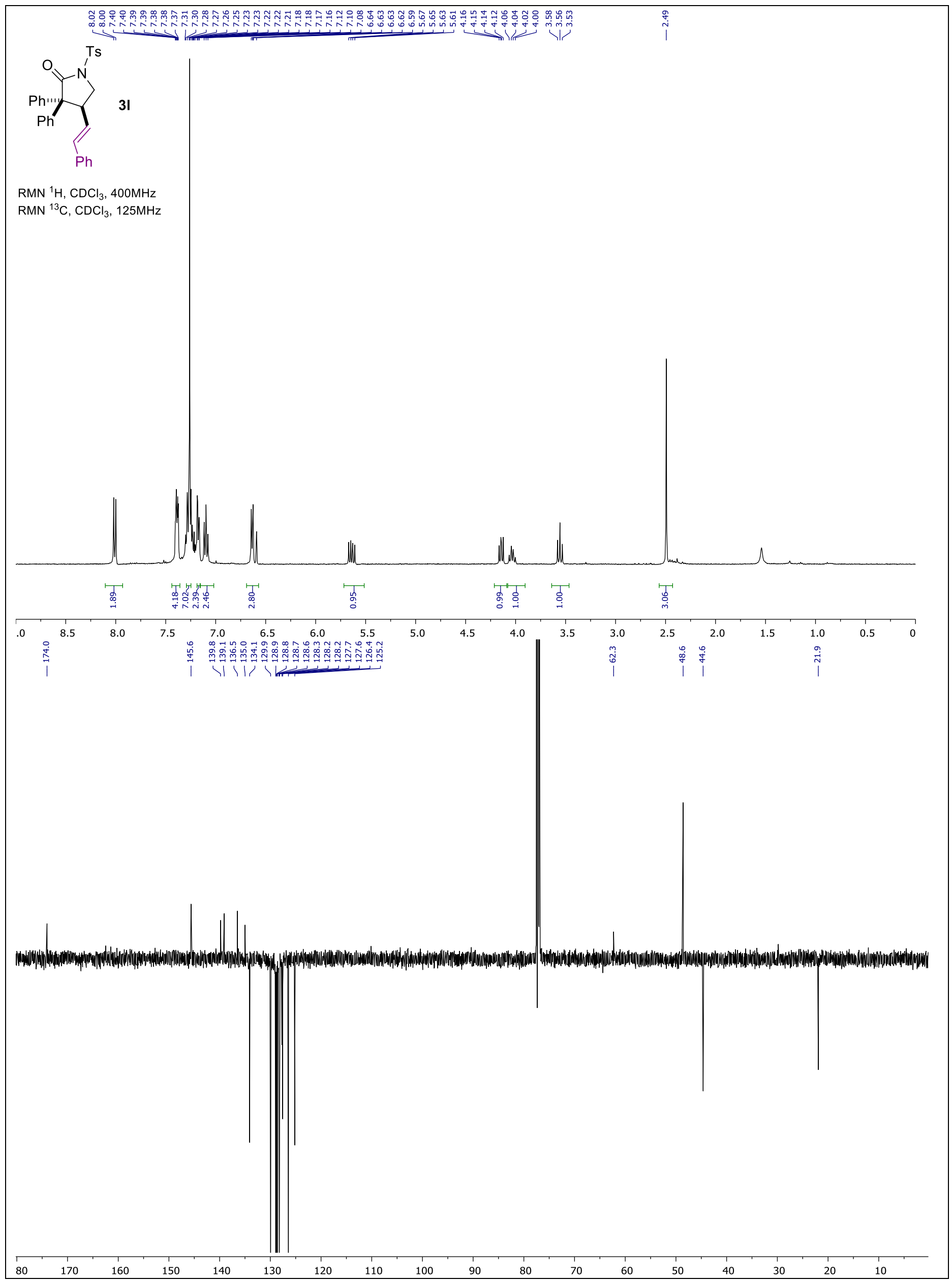




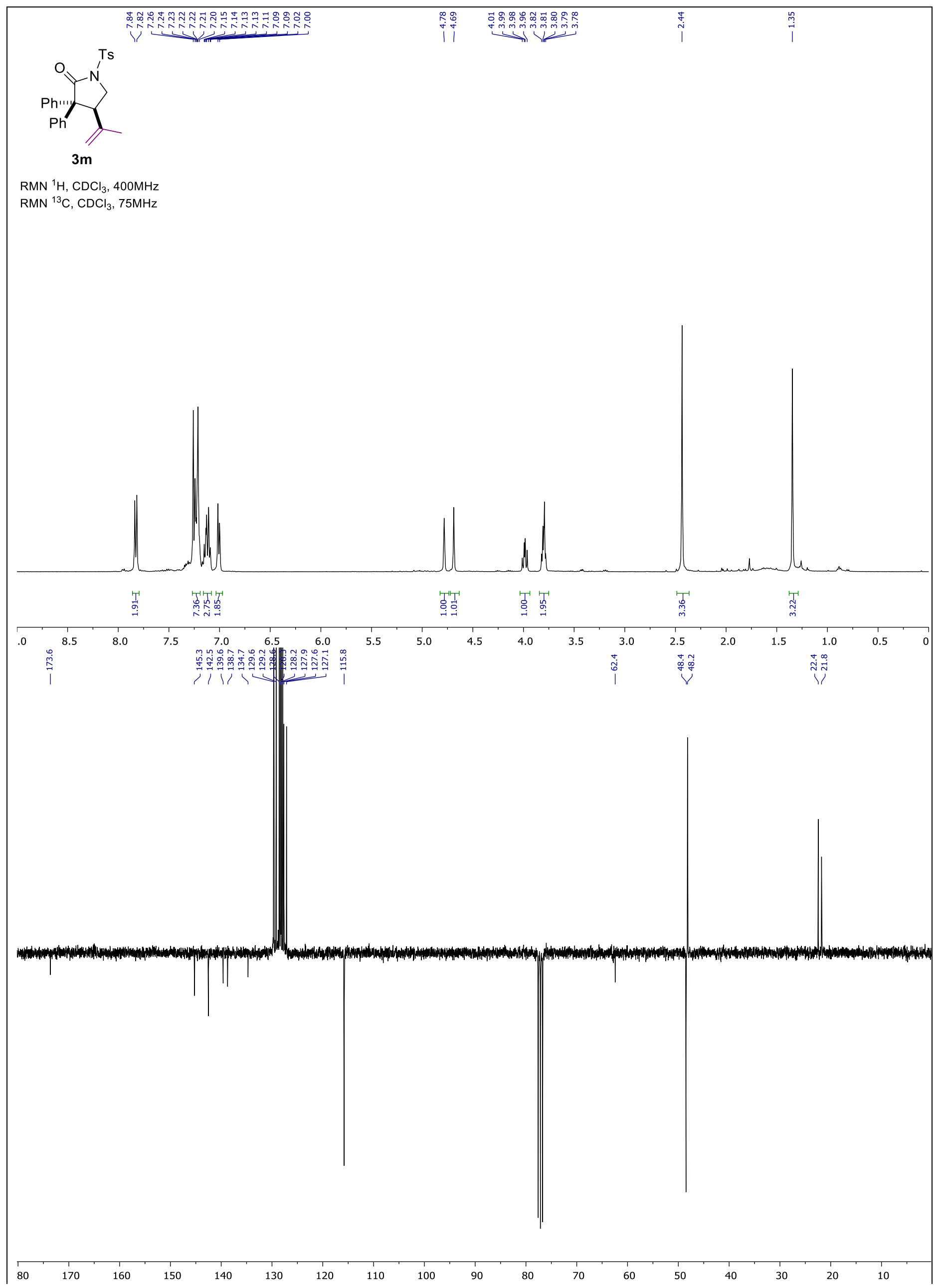




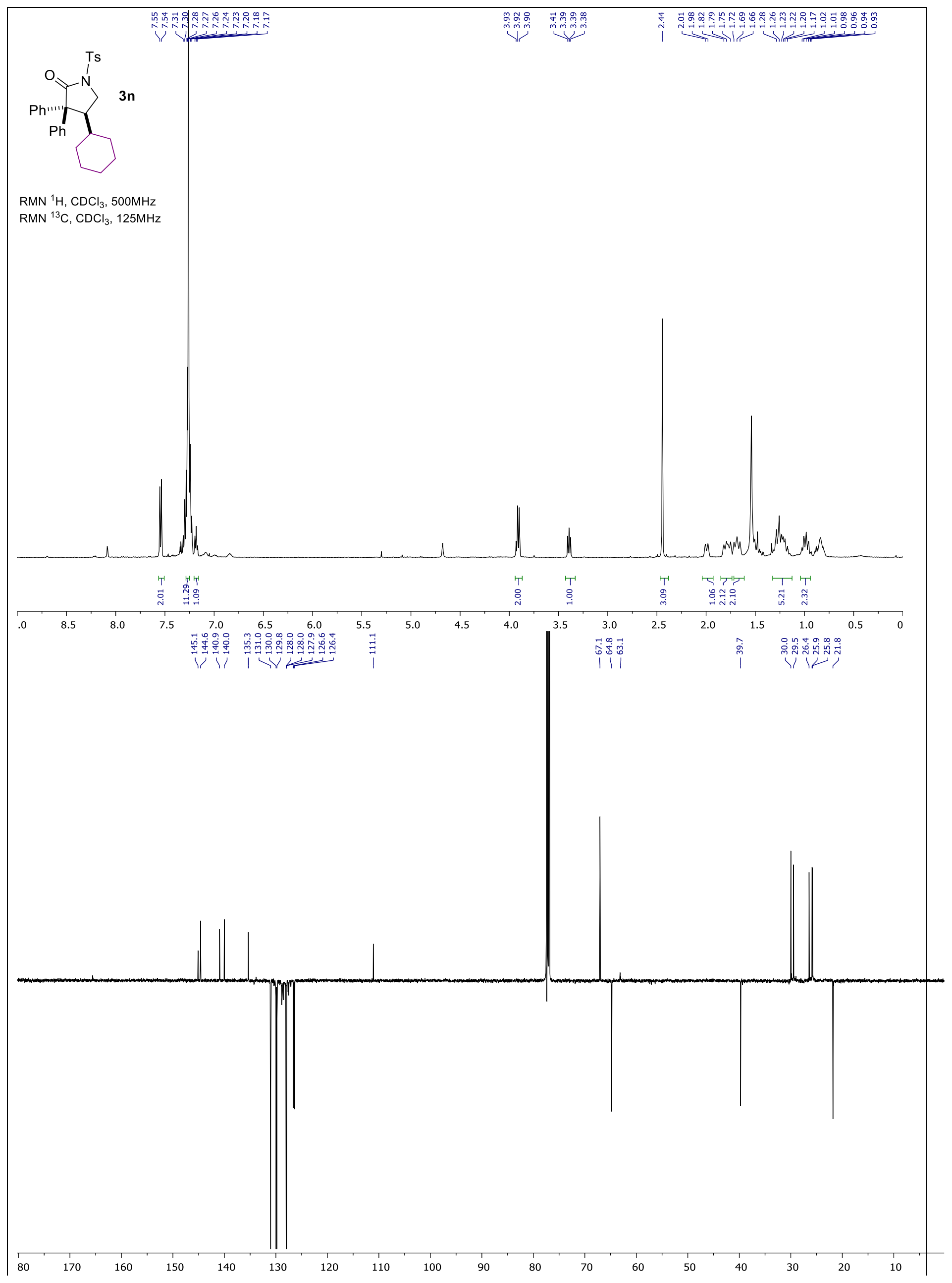




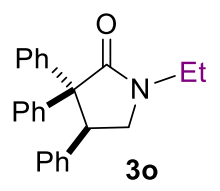

$\mathrm{RMN}{ }^{1} \mathrm{H}, \mathrm{CDCl}_{3}, 500 \mathrm{MHz}$

RMN ${ }^{13} \mathrm{C}, \mathrm{CDCl}_{3}, 125 \mathrm{MHz}$
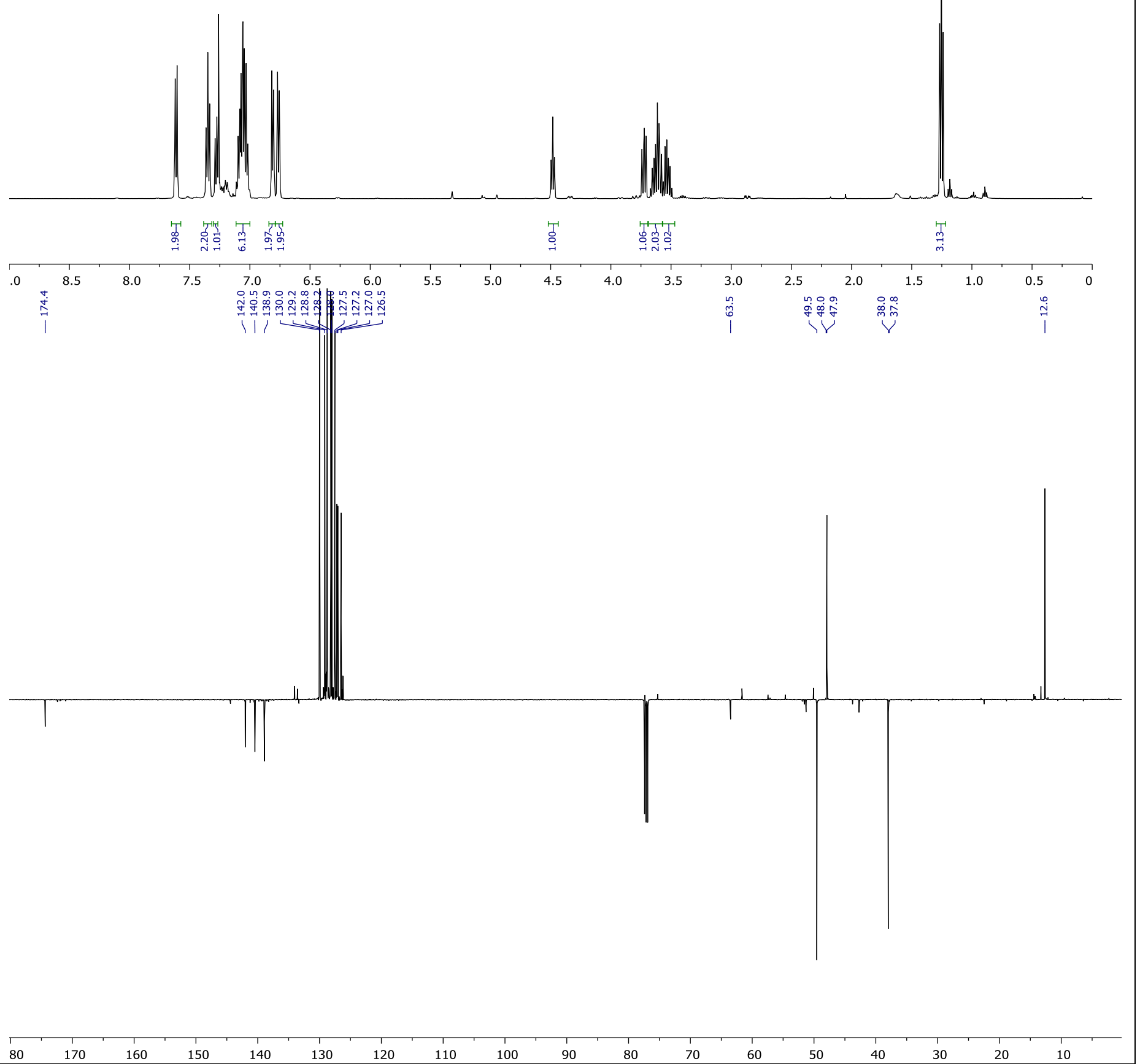


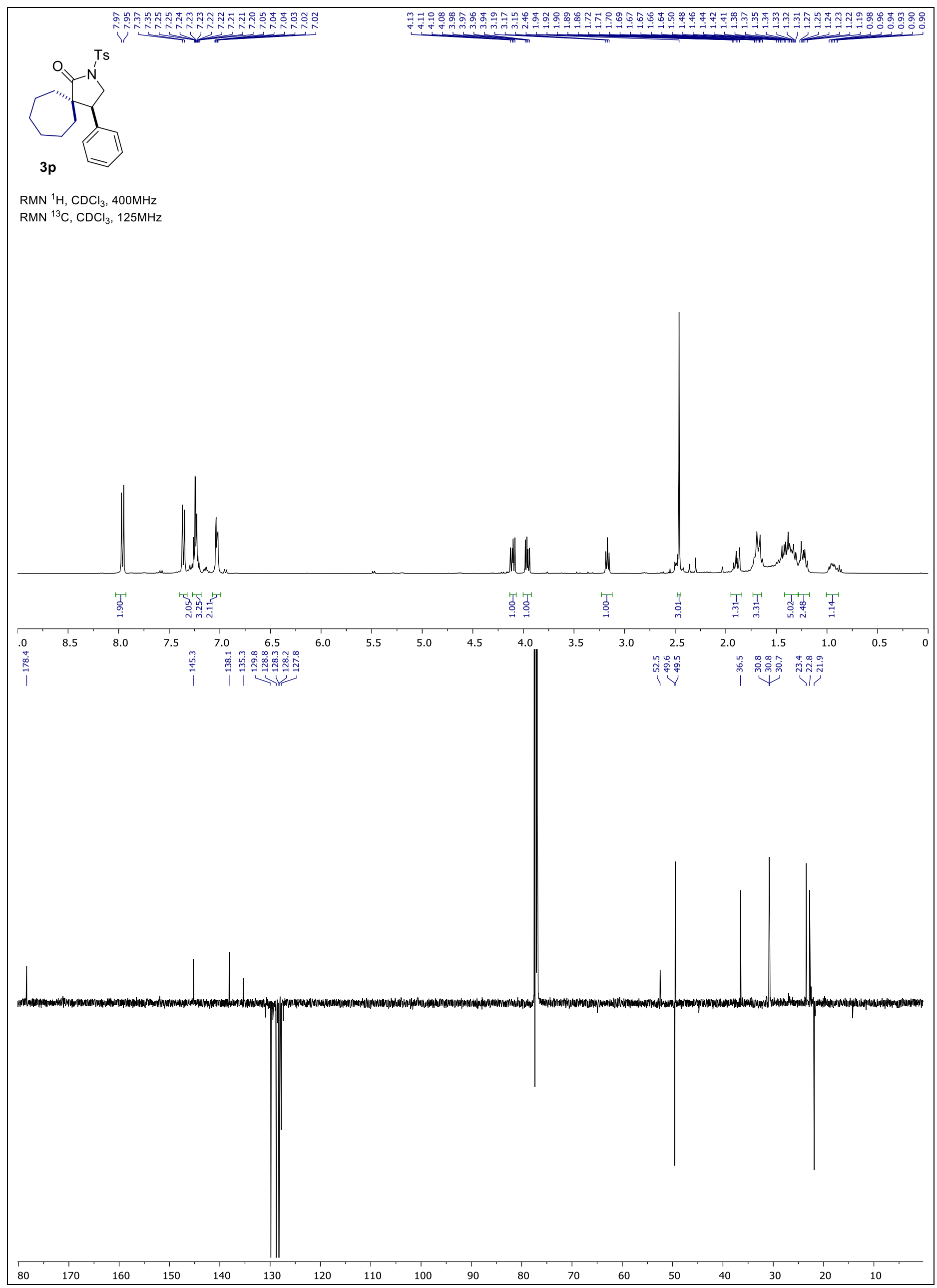




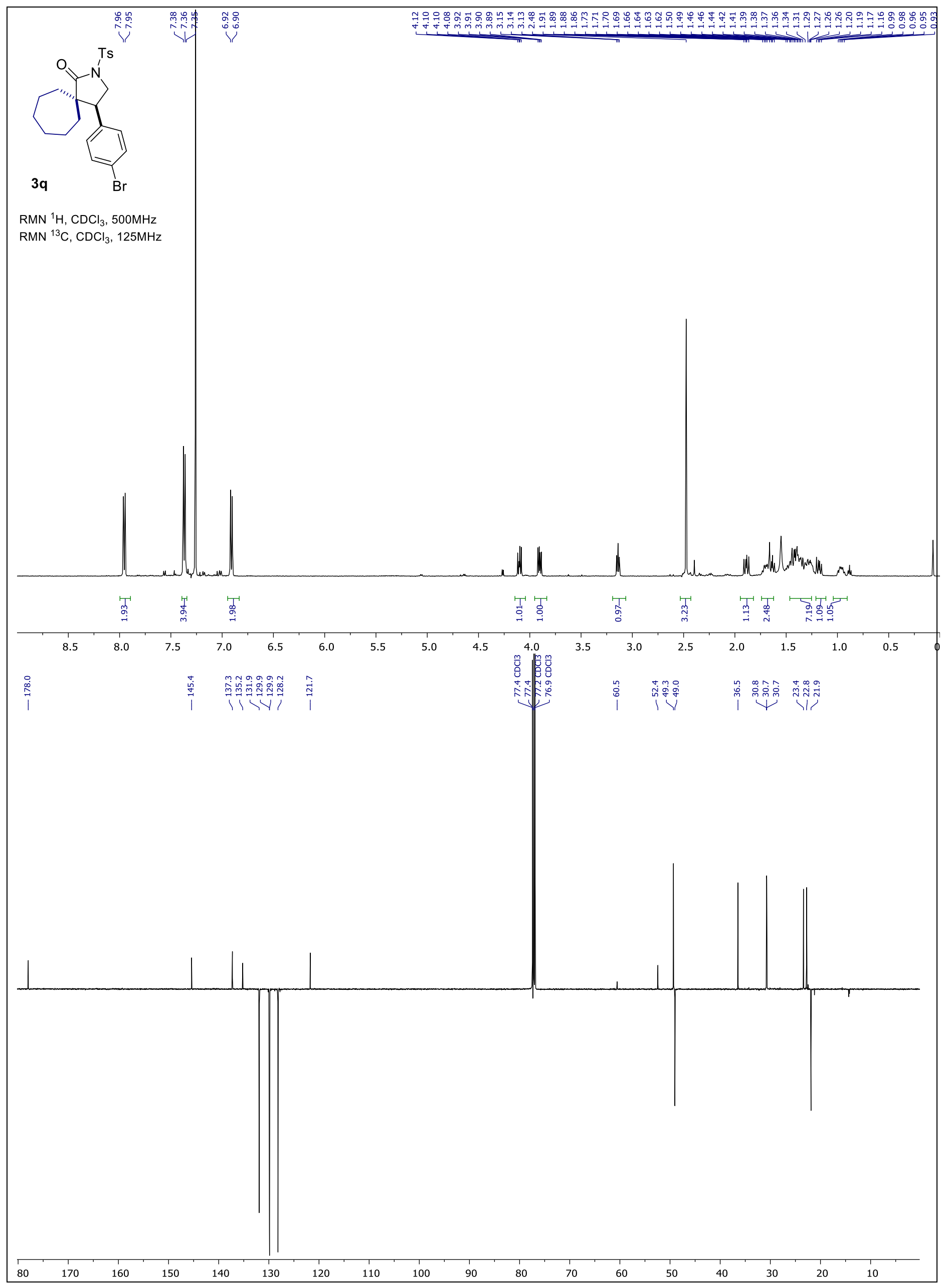




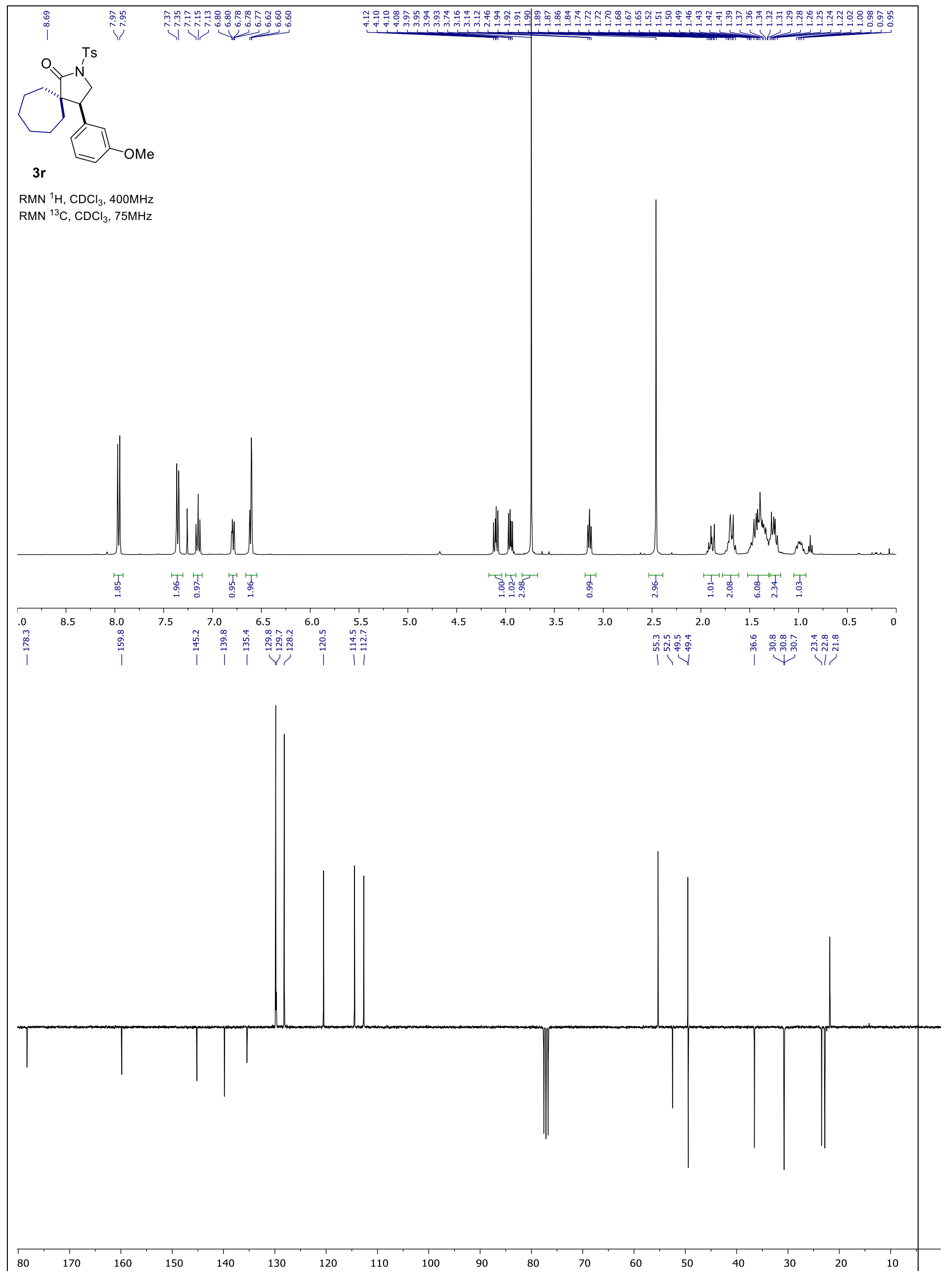




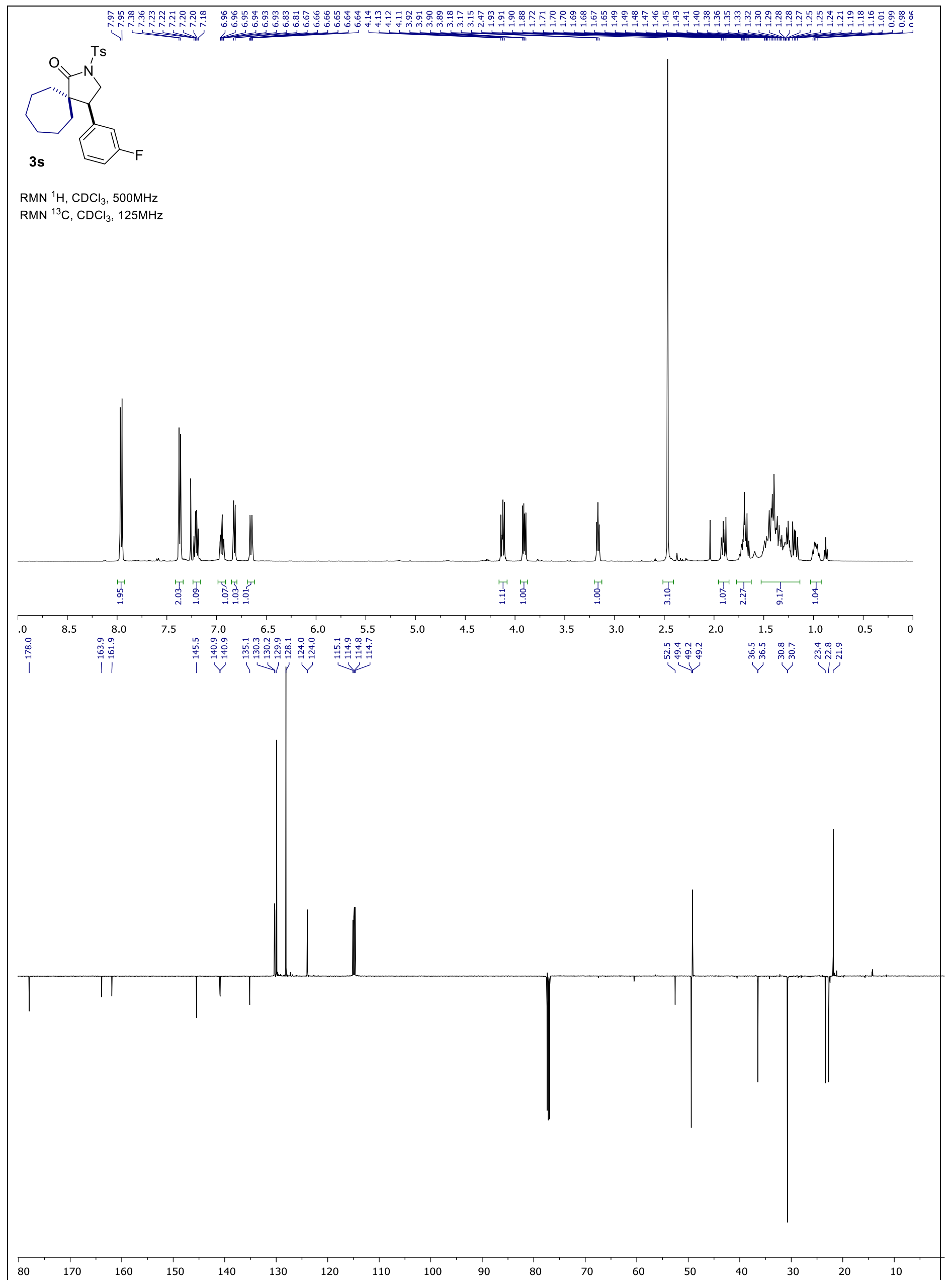




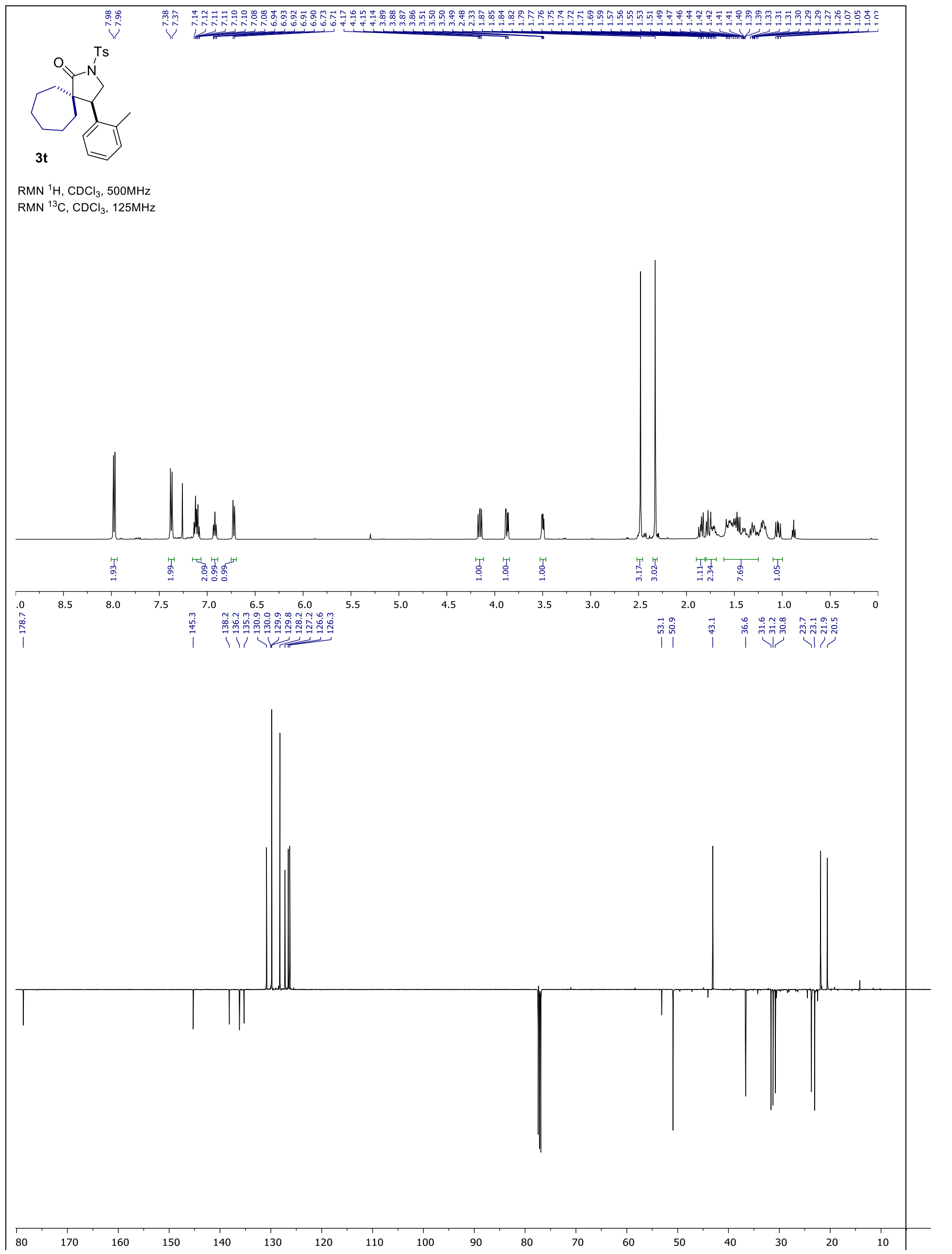




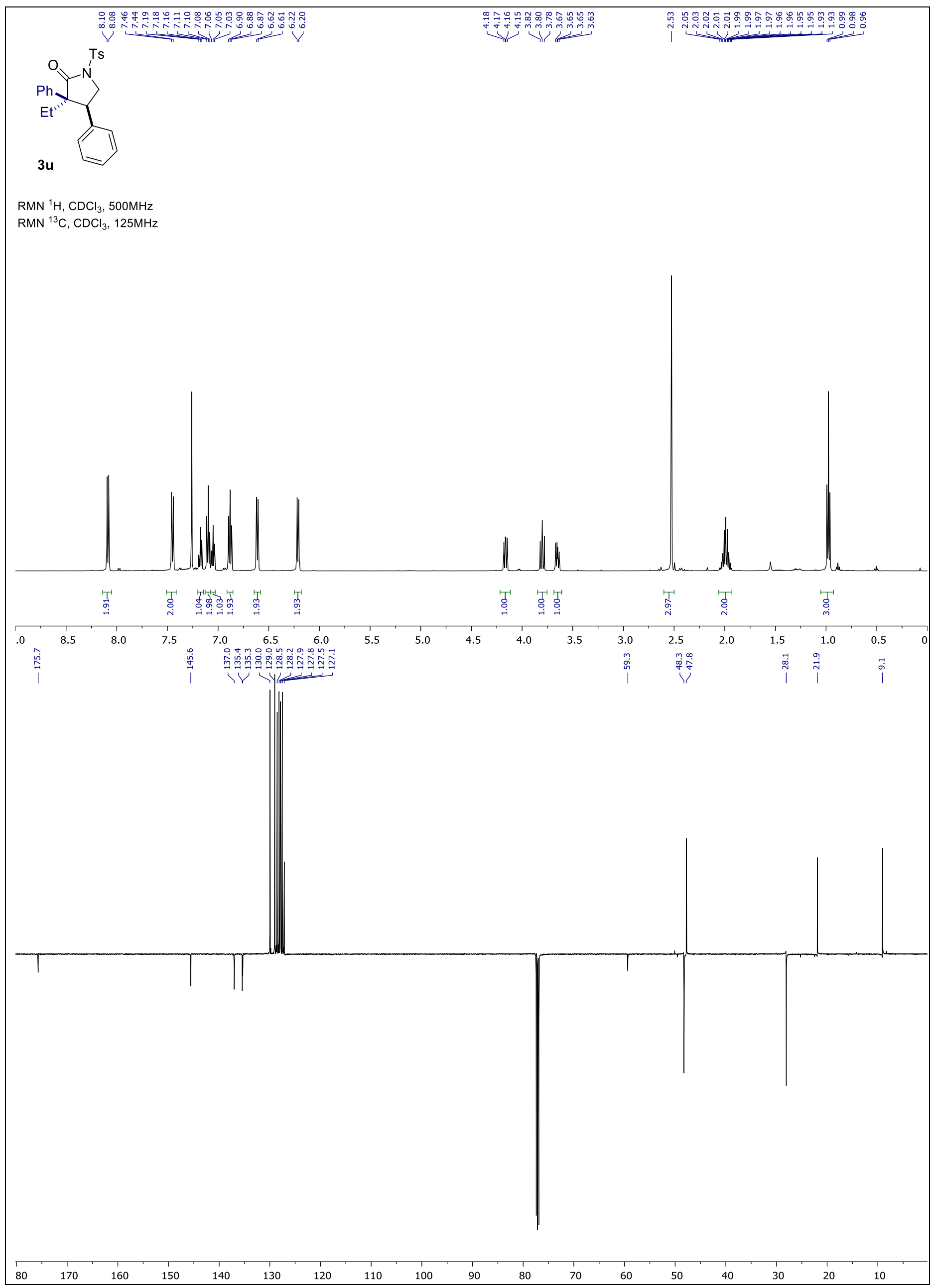




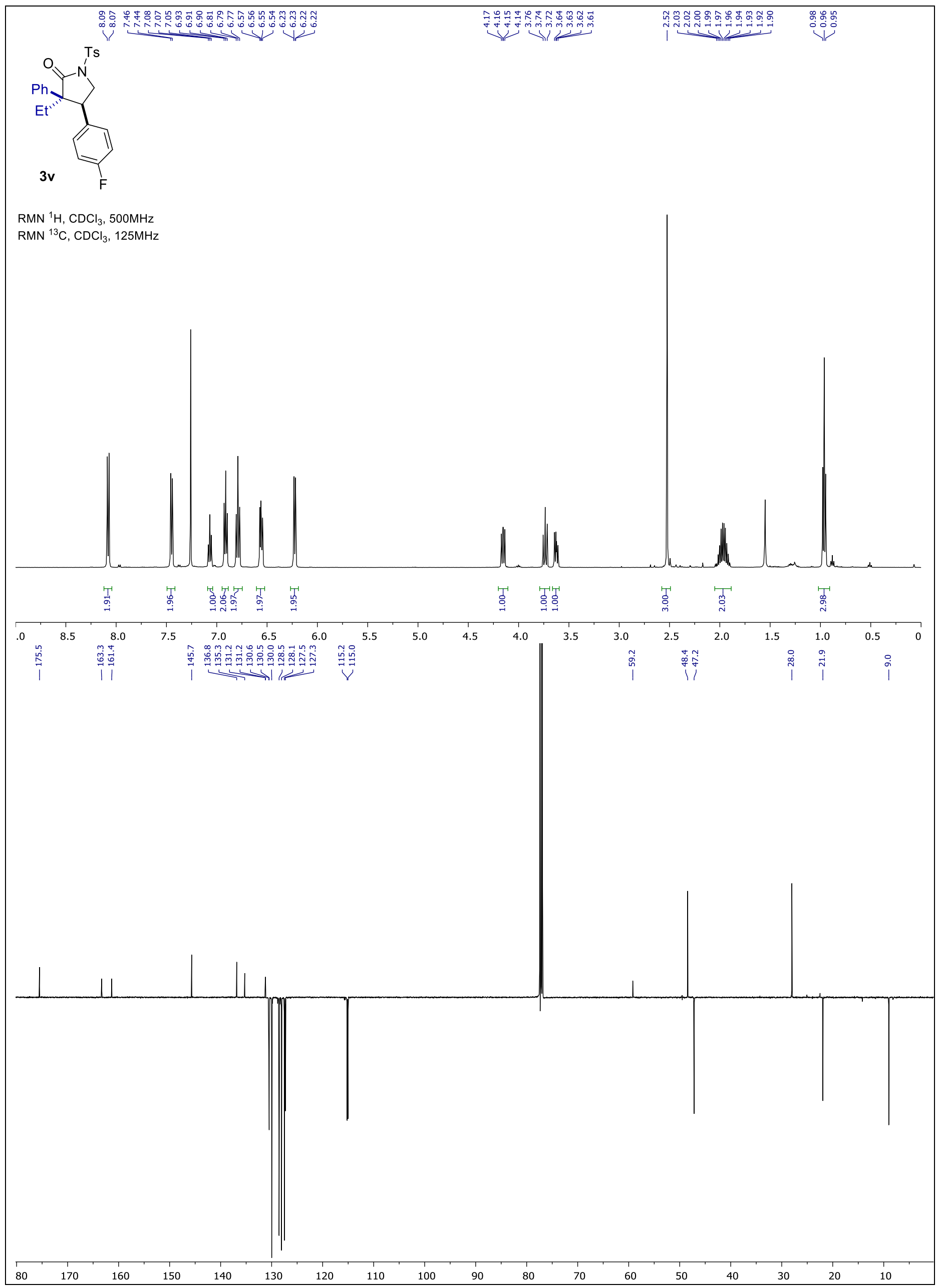




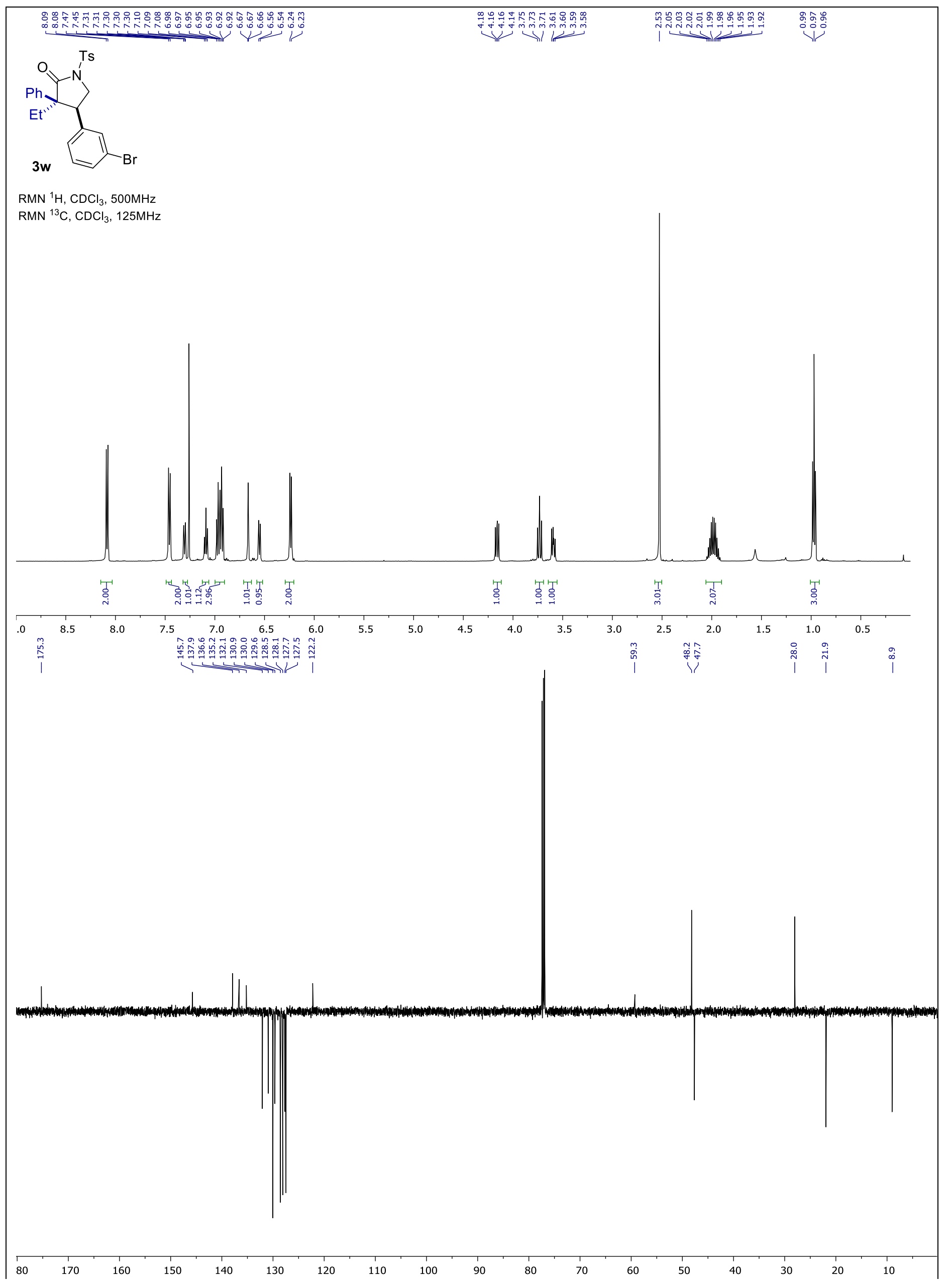




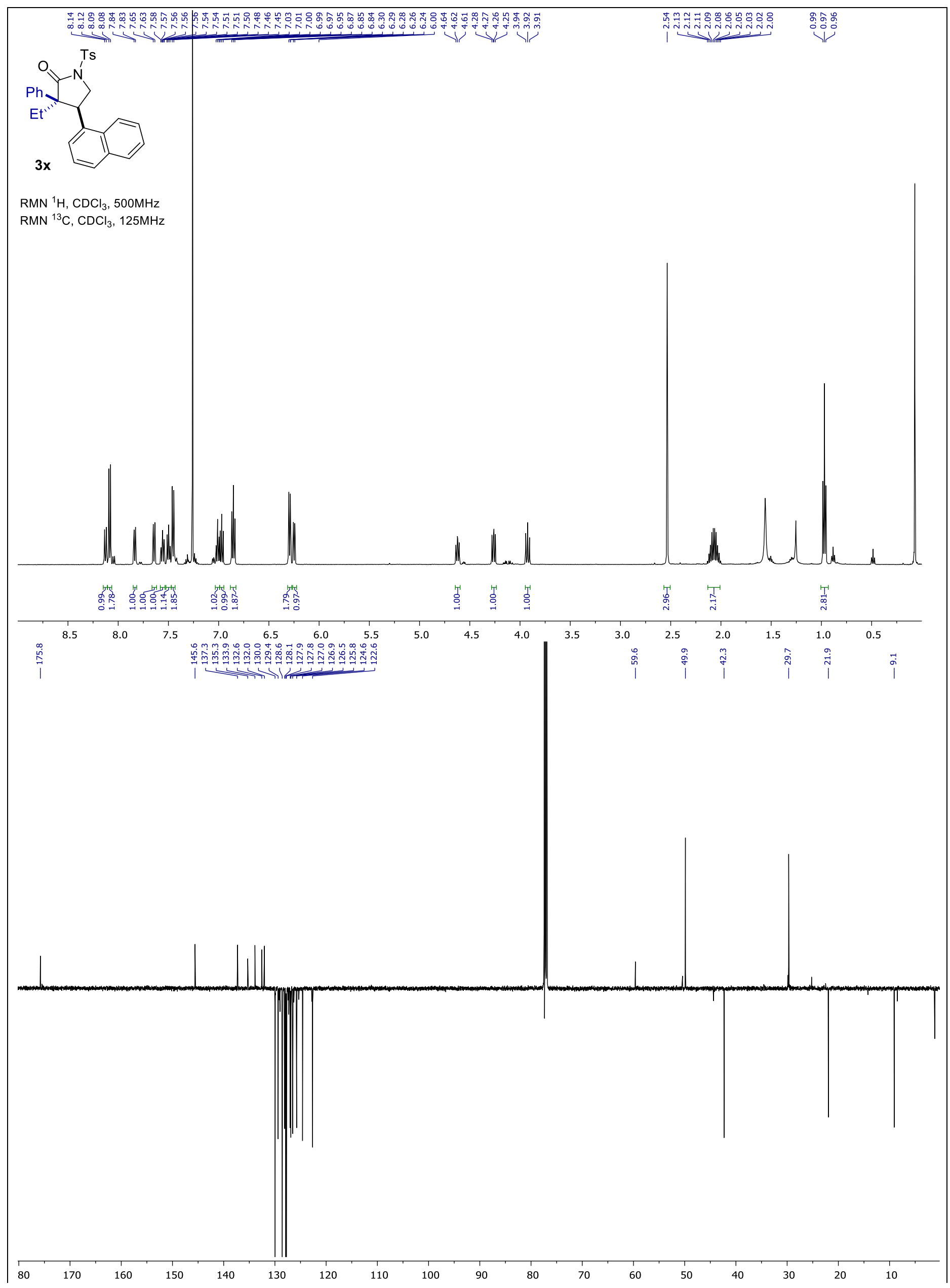




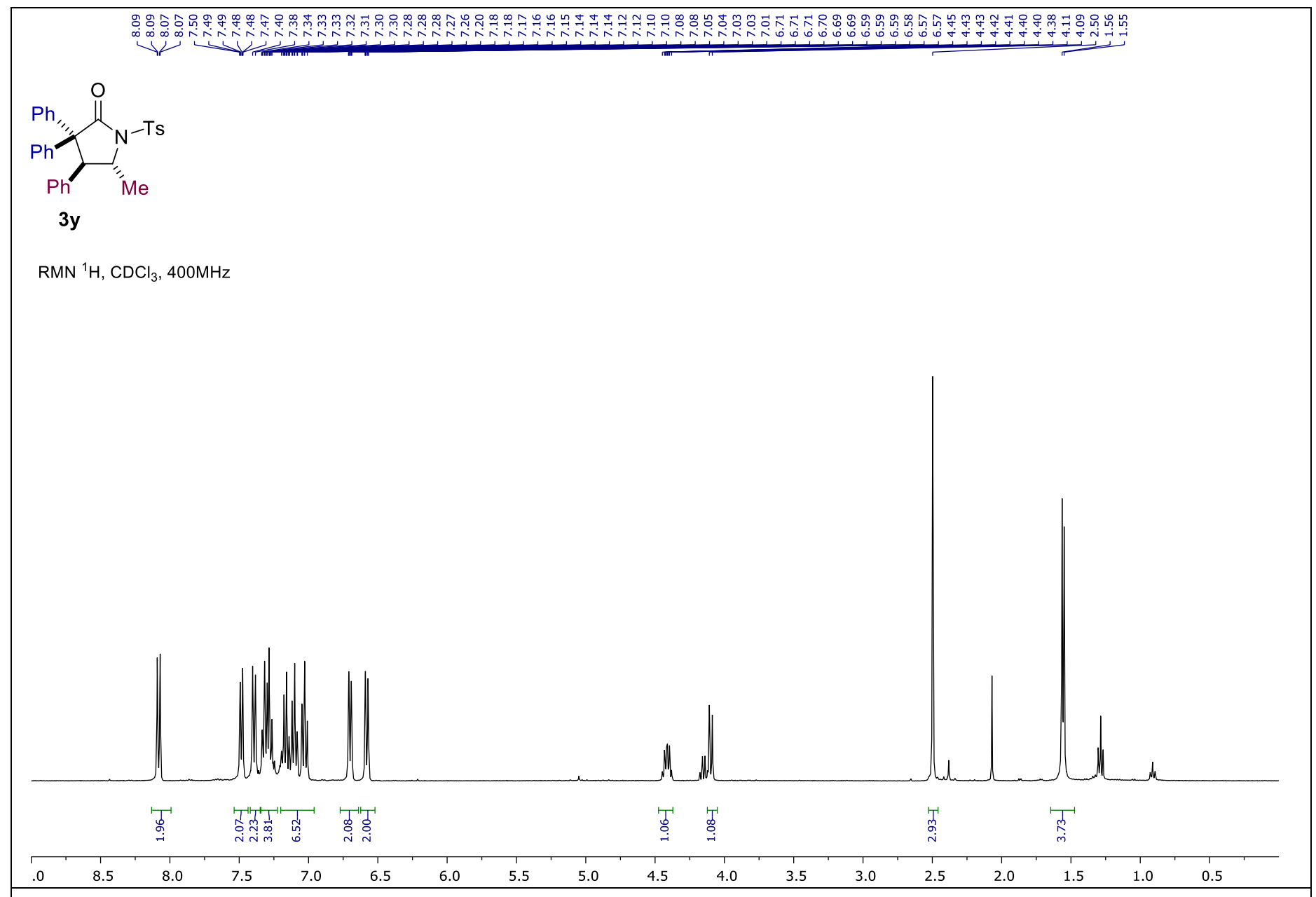

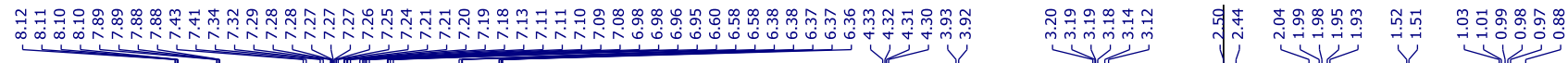

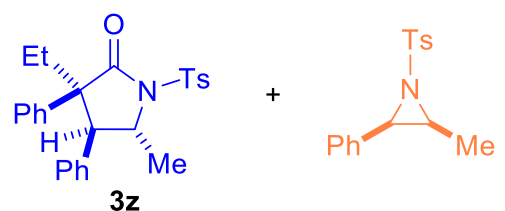

$\mathrm{RMN}{ }^{1} \mathrm{H}, \mathrm{CDCl}_{3}, 400 \mathrm{MHz}$

(crude NMR)

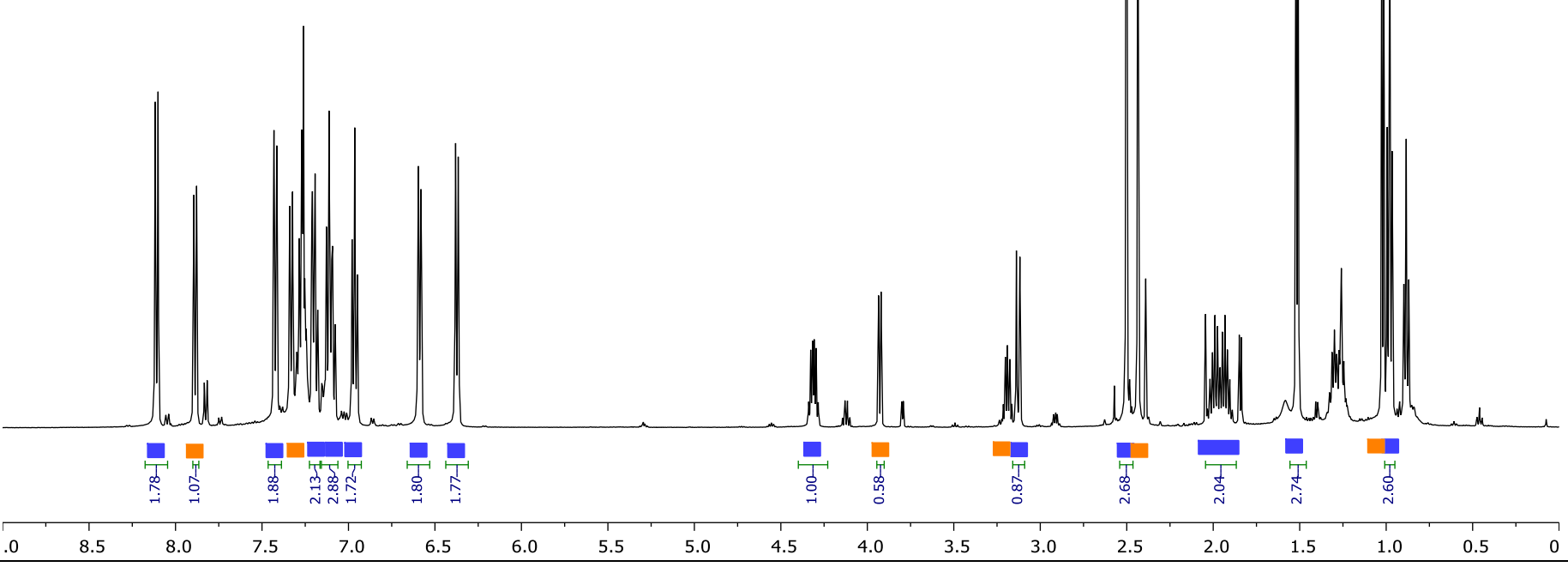




\section{HPLC Data}

Agilent 1260/1290 Infinity/Aurora SFC system. (Chiralpak IB, $s \mathrm{CO}_{2}$-methanol 80/20, flow rate $=1.7 \mathrm{~mL} / \mathrm{min}, \mathrm{I}=250$ $\mathrm{nm}) \mathrm{tR}=7.16 \mathrm{~min}, 7.76 \mathrm{~min}$.

\section{Racemic 3a}

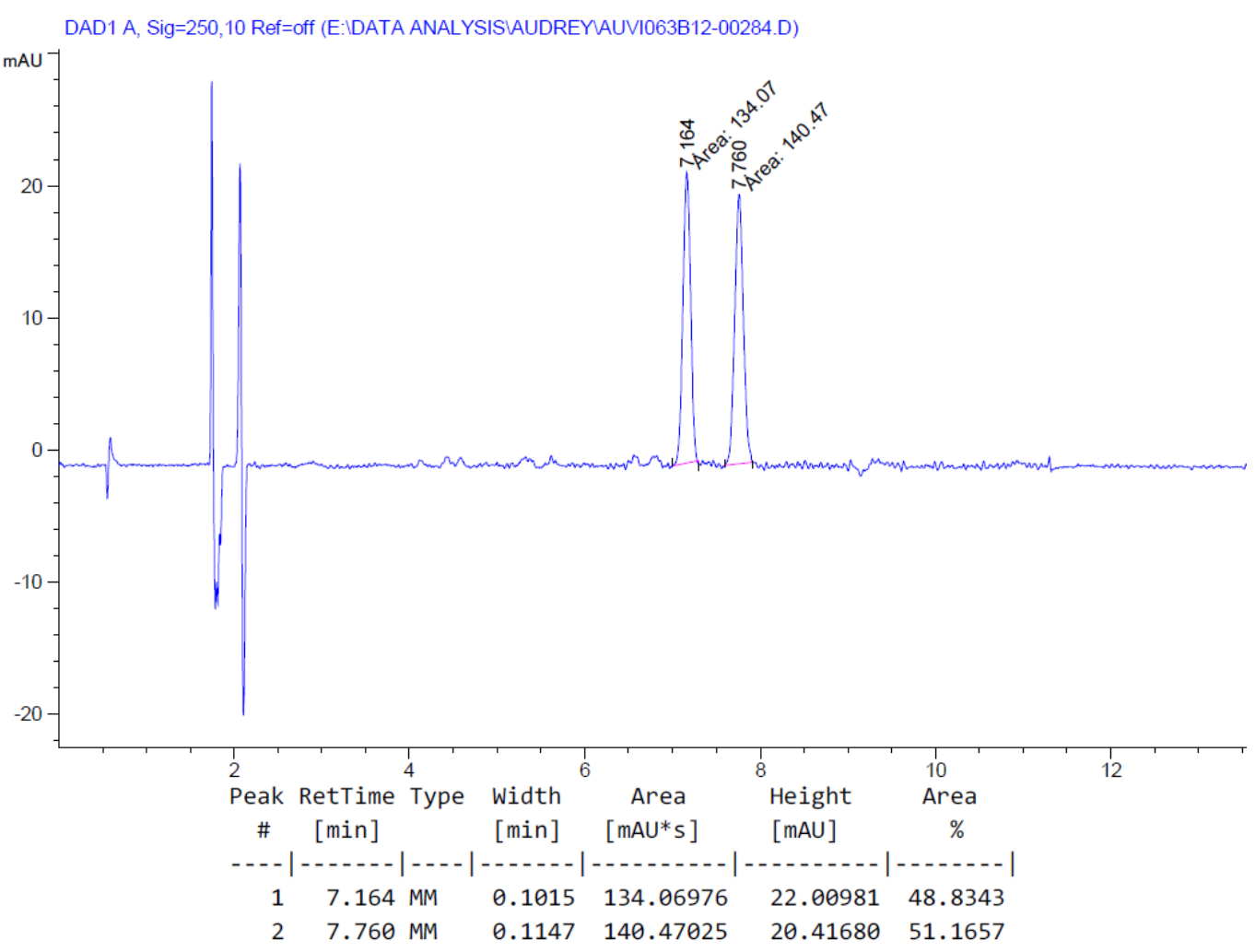

Enantioenriched (-)-3a

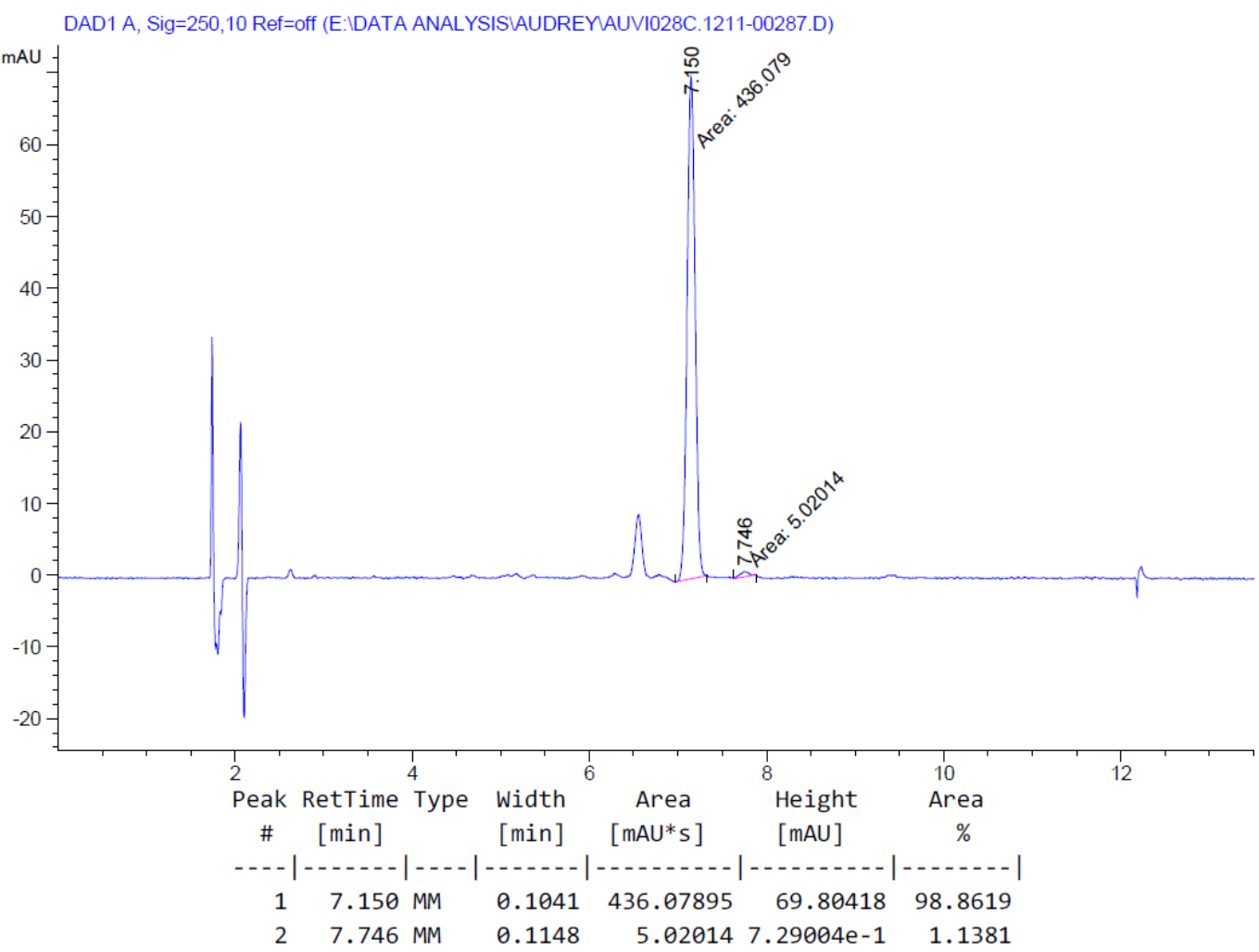




\section{X-Ray crystallographic analysis for compouds (S)-3a and 3u}

For compounds $(S)-\mathbf{3 a}$ and $\mathbf{3 u}$, measurements were made at $200 \mathrm{~K}$ on a Bruker- AXS-Enraf-Nonius KappaCCD equipped with a high brilliance Mo microsource $(\lambda=0.71073 \AA)$. The data was integrated and corrected for Lorentz and polarization effects using Eval14 ${ }^{14}$, then corrected for absorption using SADABS ${ }^{15}$ and finally merged using Xprep ${ }^{16}$. Crystallographic structures were solved using direct methods implemented by Superflip. ${ }^{17}$ Refinement was performed using ShelxL-2013 ${ }^{18}$ run under Olex $2{ }^{19}$. C, N, O, and S atoms were refined anisotropically by the full matrix least-squares method. $\mathrm{H}$ atoms were set geometrically.

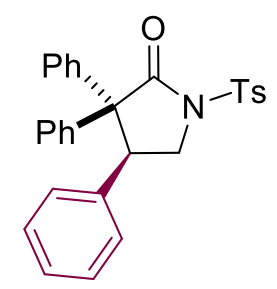

$(-)-3 a$

(S)-3,3,4-Triphenyl-1-tosylpyrrolidin-2-one (-)-3a: The title compound was recrystallized from cyclohexane/diethyl ether, by slow evaporation of solvent. Colorless crystal, mp 172-173 ${ }^{\circ} \mathrm{C} ; \mathrm{C}_{29} \mathrm{H}_{25} \mathrm{NO}_{3} \mathrm{~S}$, Monoclinic, space group P21 with $\mathrm{a}=17.989(4) \AA, \mathrm{b}=6.6711(13) \AA, \mathrm{c}=19.849(4) \AA, \beta=91.90(3)^{\circ}, \mathrm{V}=$ 2380.8(8) $\AA^{3}, Z=4, \rho_{\text {calcd }}=1.304 \mathrm{~g} / \mathrm{cm}^{3}, \mu=0.168 \mathrm{~mm}^{-1}$, Crystal size $(\mathrm{mm}): 0.32$ x $0.14 \times 0.06$. Wavelength: $\lambda$ Mo K $\alpha=0.71073 \AA, \Theta_{\max } 25$. Total and independent reflections: 30461 and 7012, Rint : 0.0615, R=0.0418, $\mathrm{wR}=0.0950$, residual electronic density: 0.160, Flack parameter: -0.14(5). CCDC deposition Number 1998270 contains the supplementary crystallographic data for this structure. These data are provided free of charge by the joint Cambridge Crystallographic Data Centre and Fachin formations zentrum Karlsruhe Access Structures service www.ccdc.cam.ac.uk/structures.

\footnotetext{
${ }^{14}$ Duisenberg, A. J. M., Kroon-Batenburg, L. M. J. \& Schreurs, A. M. M. (2003). J. Appl. Cryst. 36, $220-229$.

${ }^{15}$ Bruker (2004). SADABS. Bruker AXS Inc., Madison, Wisconsin, USA.

${ }^{16}$ Bruker (2005). XPREP. Bruker AXS Inc., Madison, Wisconsin, USA.

${ }^{17}$ Palatinus, L. \& Chapuis, G. (2007). J. Appl. Cryst. 40, 786-790.

${ }^{18}$ Sheldrick, G. M. (2015). Acta Cryst. C71, 3-8.

${ }^{19}$ Dolomanov, O.V., Bourhis, L.J., Gildea, R.J, Howard, J.A.K. \& Puschmann, H. (2009), J. Appl. Cryst. 42, 339-341
} 

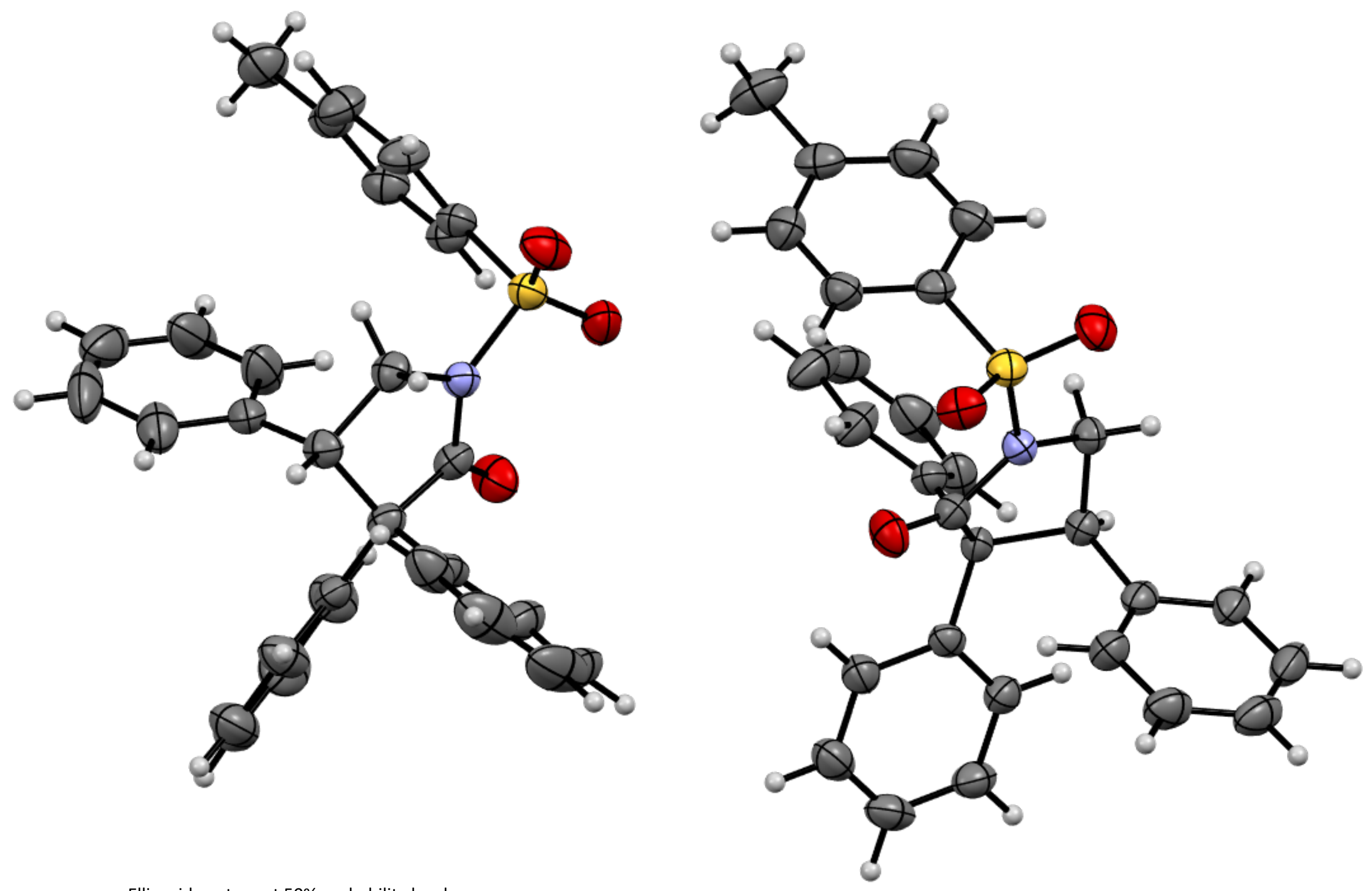

Ellipsoid contour at $50 \%$ probability levels 


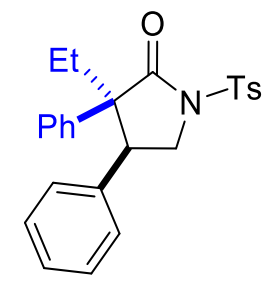

$3 u$

3-Ethyl-3,4-diphenyl-1-tosylpyrrolidin-2-one (3u): The title compound was recrystallized from cyclohexane/diethyl ether, by slow evaporation of solvent. Colorless crystal, mp 141-142 ${ }^{\circ} \mathrm{C} ; \mathrm{C}_{25} \mathrm{H}_{25} \mathrm{NO}_{3} \mathrm{~S}$, Monoclinic, space group P2 $1 / \mathrm{n}$ with $\mathrm{a}=9.822(2) \AA, \mathrm{b}=8.0923(16) \AA, \mathrm{c}=27.508(6) \AA, \beta=91.40(3)^{\circ}, \mathrm{V}=$ 2185.8(8) $\AA^{3}, Z=4, \rho_{\text {calcd }}: 1.275 \mathrm{~g} / \mathrm{cm}^{3}, \mu: 0.174 \mathrm{~mm}^{-1}$, Crystal size $(\mathrm{mm}): 0.44$ x 0.16 x 0.03 . Wavelength: $\lambda$ Mo K $\alpha=0.71073 \AA, \Theta_{\max } 25^{\circ}$. Total and independent reflections: 17155 and 3835, Rint : 0.0385, $\mathrm{R}=$ 0.0585, wR $=0.1364$, residual electronic density: 0.302. CCDC deposition Number 1998271 contains the supplementary crystallographic data for this structure. These data are provided free of charge by the joint Cambridge Crystallographic Data Centre and Fachinformationszentrum Karlsruhe Access Structures service www.ccdc.cam.ac.uk/structures. 


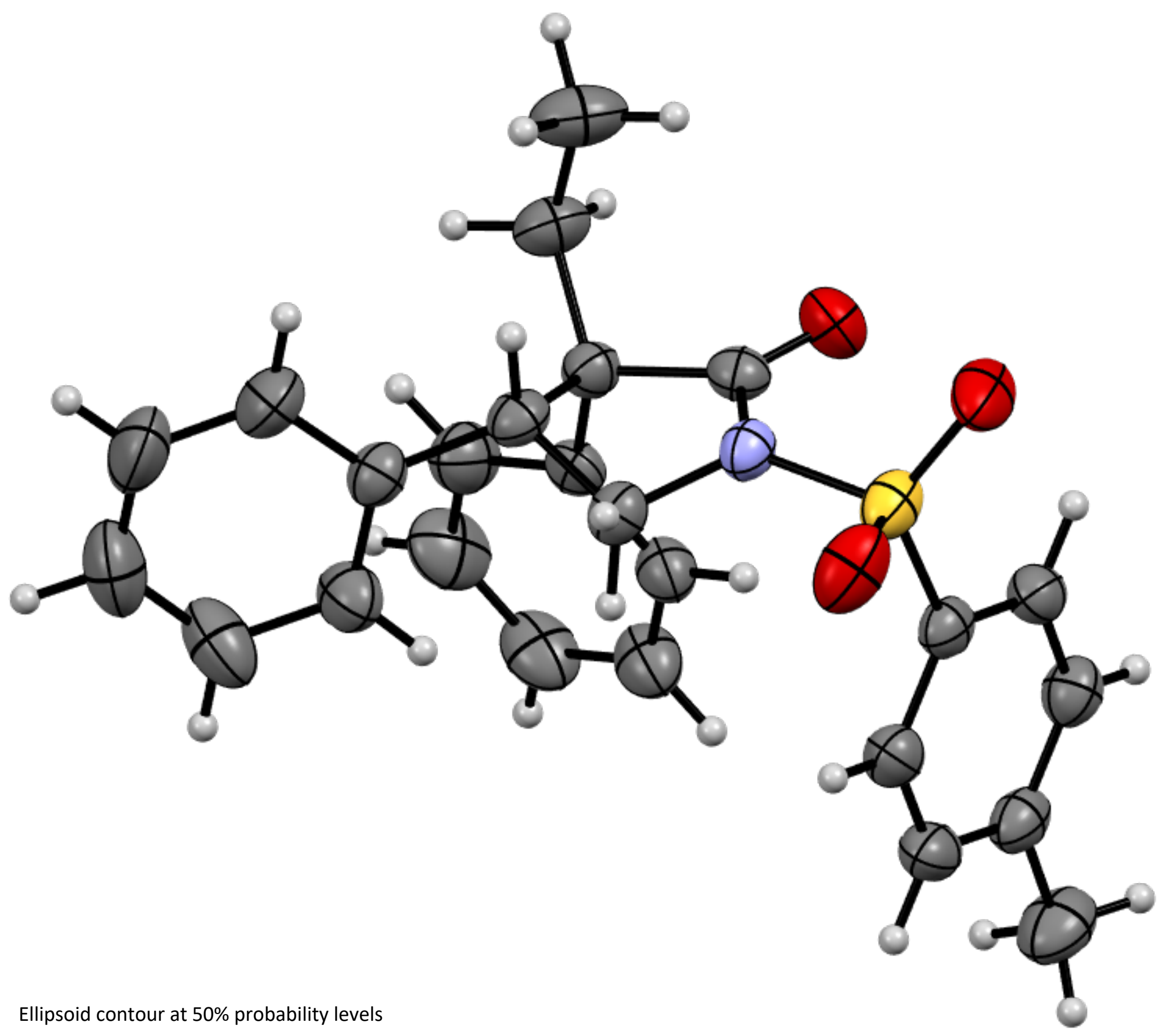

